Supporting Information

\title{
Josiphos-type Binaphane Ligands for Iridium-Catalyzed Enantioselective Hydrogenation of 1-Aryl-Substituted Dihydroisoquinolines
}

\author{
Huifang Nie, ${ }^{\dagger}$ Yupu Zhu, ${ }^{\dagger}$ Xiaomu Hu, ${ }^{\dagger}$ Zhao Wei, ${ }^{\dagger}$ Lin Yao,${ }^{\dagger}$ Gang Zhou, ${ }^{\dagger}$ \\ Pingan Wang, ${ }^{, \dagger}$ Ru Jiang, ${ }^{*},+$ and Shengyong Zhang ${ }^{*, \dagger}$ \\ * Corresponding authors
}

School of Pharmacy, Fourth Military Medical University, Xi'an, 710032, China E-mail: syzhang@fmmu.edu.cn

\section{Contents}

$\begin{array}{ll}\text { 1. General remarks } & \text { S2 }\end{array}$

$\begin{array}{ll}\text { 2. General procedure for synthesis of ligands } & \text { S2 }\end{array}$

3. General procedure for synthesis of 1-aryl-3,4-DHIQs $\quad$ S7

4. Optimization of AH reaction conditions $\quad \mathrm{S} 15$

5. General procedure for AH and scaled-up catalytic hydrogenation $\quad$ S16

$\begin{array}{ll}\text { 6. NMR spectra and HRMS } & \text { S26 }\end{array}$

$\begin{array}{ll}\text { 7. HPLC spectra } & \text { S66 }\end{array}$ 


\section{General remarks}

(R)-Ugi's amine (2) was purchased from XylMED Biomedical (Xi'an, China). All other commercial chemicals were purchased from $J \& K$ or Energy Chemical (Shanghai, China) in the highest purity and used without purification. All reactions and manipulations were performed using standard Schlenk techniques. THF, $t$-butyl methyl ether (TBME) and toluene were distilled from sodium benzophenone ketyl. $\mathrm{CH}_{2} \mathrm{Cl}_{2}, \mathrm{CHCl}_{3}$ and ethyl acetate were distilled form $\mathrm{CaH}_{2}$ under an atmosphere of argon. NMR spectra were recorded on a Bruker $400 \mathrm{MHz}$ NMR spectrometer. Optical rotations analyses were performed on a Perkin-Elmer Model 343 Polarimeter. HRMS were recorded on ZAB-HS spectrometer with ES ionization (ESI). HPLC analysis was performed on an Agilent 1260 Infinity instrument with a chiral stationary phase using a Daicel Chiralcel OD-H column or a Daicel Chiralcel AD-H column ( $n$-hexane/iso-propanol mixtures as solvent).

\section{General procedure for synthesis of ligands ${ }^{1-3}$}

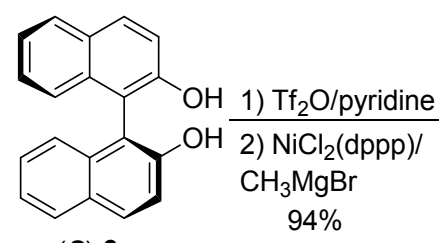

(S)-3

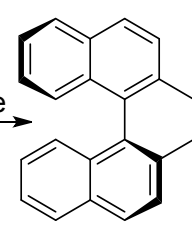

(S)-4

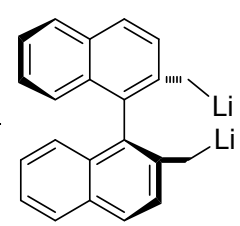

(S)-5
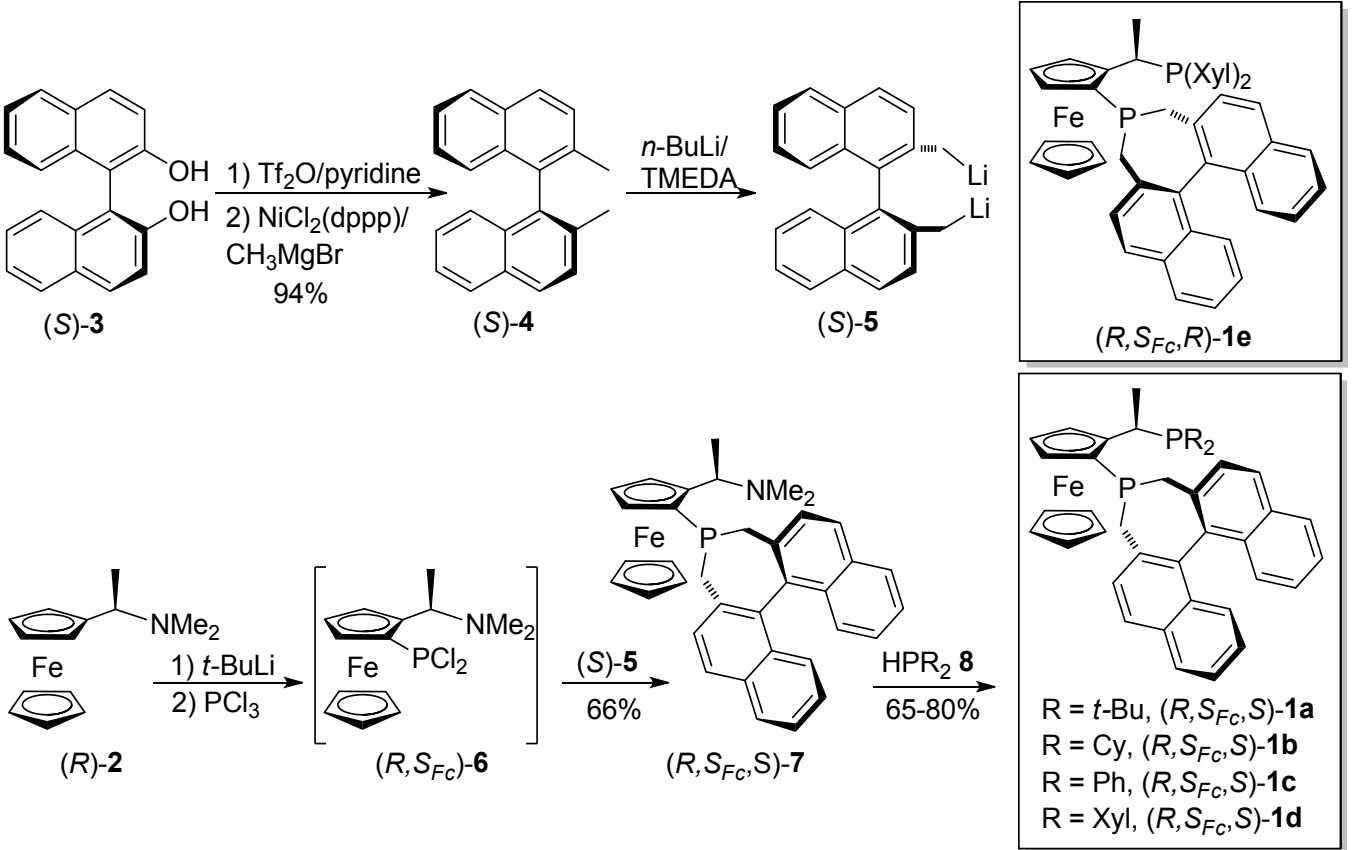
(S)-4. To a solution of $(S)-3(10 \mathrm{~g}, 36 \mathrm{mmol})$ in $\mathrm{CH}_{2} \mathrm{Cl}_{2}(50 \mathrm{~mL})$ at $0{ }^{\circ} \mathrm{C}$ were added pyridine ( $8.5 \mathrm{~g}, 108 \mathrm{mmol}, 3.0$ equiv) and subsequently trifluoromethanesulfonic anhydride ( $22 \mathrm{~g}, 78 \mathrm{mmol}, 2.2$ equiv). The reaction was allowed to warm to room temperature and stirred for $5 \mathrm{~h}$. The reaction was quenched by the addition of water $(100 \mathrm{~mL})$, and the organic phases were separated. The aqueous layer was further extracted with $\mathrm{CH}_{2} \mathrm{Cl}_{2}(2 \times 50 \mathrm{~mL})$. The combined organic phases were washed with brine $(100 \mathrm{~mL})$, dried over $\mathrm{Na}_{2} \mathrm{SO}_{4}$, and concentrated under reduced pressure. The crude product was used in the next step without further purification.

1,3-Bis-(diphenylphosphino)propane nickel (II) chloride (976 mg, $1.8 \mathrm{mmol}, 5$ mol \%) was placed in a flame-dried Schlenk flask, the above prepared product (36 mmol) in TBME $(150 \mathrm{~mL})$ was added. The suspension was cooled to $0{ }^{\circ} \mathrm{C}$, and methylmagnesium bromide (3.0 $\mathrm{M}$ in $\mathrm{Et}_{2} \mathrm{O}, 36 \mathrm{~mL}, 108 \mathrm{mmol}, 3.0$ equiv) was added dropwise. The cooling bath was removed and the reaction was stirred at room temperature overnight. The mixture was cooled to $0{ }^{\circ} \mathrm{C}$ and was carefully quenched by the addition of hydrochloric acid $(2 \mathrm{M}, 35 \mathrm{~mL})$. The phases were separated, and the aqueous phase was extracted with TBME $(2 \times 100 \mathrm{~mL})$. The combined organic extracts were washed with brine, dried over $\mathrm{Na}_{2} \mathrm{SO}_{4}$, and concentrated under reduced pressure. The residue was purified by flash column chromatography on silica gel using PE as eluent, affording (S)-4 (9.54 g, $33.8 \mathrm{mmol})$ as a white solid (yield: $94 \%$ ). Characterization of $(S)-\mathbf{4}:{ }^{1} \mathbf{H}$ NMR $\left(400 \mathrm{MHz}, \mathrm{CDCl}_{3}\right) \delta 7.88(\mathrm{t}, J=7.1 \mathrm{~Hz}, 4 \mathrm{H})$, $7.50(\mathrm{~d}, J=8.3 \mathrm{~Hz}, 2 \mathrm{H}), 7.38(\mathrm{t}, J=7.4 \mathrm{~Hz}, 2 \mathrm{H}), 7.19(\mathrm{t}, J=7.6 \mathrm{~Hz}, 2 \mathrm{H}), 7.04(\mathrm{~d}, J=$ $8.4 \mathrm{~Hz}, 2 \mathrm{H}), 2.03(\mathrm{~s}, 6 \mathrm{H})$.

$\left(R_{C}, S_{F c}, S_{a x}\right)$-7. A solution of $n$-BuLi (1.6 M in $n$-hexane, $32 \mathrm{~mL}, 51.2 \mathrm{mmol}, 2.5$ equiv) was concentrated under vacuum and the resulting oil was dissolved in TBME $(15 \mathrm{~mL})$. A solution of $(S)-4(5.64 \mathrm{~g}, 20 \mathrm{mmol})$ in TBME $(40 \mathrm{~mL})$ was added over a dropping funnel during $20 \mathrm{~min}$ to give a red solution. Afterwards, TMEDA $(7.8 \mathrm{~mL}$, $52 \mathrm{mmol}$, distilled over $\mathrm{CaH}_{2}$ ) was added slowly, and the resulting solution was stirred for $24 \mathrm{~h}$ at room temperature. The supernatant solution was decanted via a tube. The crystals were washed twice with dry $n$-hexane $(10 \mathrm{~mL}$, removed by a tube) 
and dried under vacuum to give dilithium salt $5(3.53 \mathrm{~g}, 12 \mathrm{mmol})$ as deep red crystals (yield: $60 \%$ ).

To a solution of $(R)$-Ugi's amine (2) (2.57 g, $10 \mathrm{mmol})$ in TBME (20 mL) was added $t$-BuLi (1.3 $\mathrm{M}$ in $n$-pentane, $8.5 \mathrm{~mL}, 11 \mathrm{mmol}, 1.1$ equiv) at $0{ }^{\circ} \mathrm{C}$. After addition was completed, the mixture was warmed to room temperature, and stirred for $1.5 \mathrm{~h}$ at room temperature. The mixture was then cooled to $-78{ }^{\circ} \mathrm{C}$ and a solution of $\mathrm{PCl}_{3}(1$ $\mathrm{mL}, 11.46 \mathrm{mmol})$ in TBME $(5 \mathrm{~mL})$ was added slowly, and the mixture was warmed to room temperature overnight to afford the dichlorophosphine $\left(R_{C}, S_{F c}\right)$-6. After the mixture was cooled to $-20^{\circ} \mathrm{C}$, a suspension of dilithium salt $5(2.94 \mathrm{~g}, 10 \mathrm{mmol})$ in TBME was added via a cannula. The mixture was stirred overnight from $-20^{\circ} \mathrm{C}$ to room temperature and quenched by the addition of saturated $\mathrm{NH}_{4} \mathrm{Cl}$ solution $(40 \mathrm{~mL})$. The organic layer was separated and dried over $\mathrm{Na}_{2} \mathrm{SO}_{4}$ and the solvent was removed under reduced pressure. The residue was purified by chromatography to afford $\left(R_{C}, S_{F c}, S_{a x}\right)-7(3.74 \mathrm{~g}, 6.6 \mathrm{mmol})$ as a yellow foam (yield: $\left.66 \%\right)$. Characterization of $\left(R_{C}, S_{F c}, S_{a x}\right)-7:[\boldsymbol{\alpha}]_{\mathbf{D}}^{25}-465.7\left(c=0.25, \mathrm{CHCl}_{3}\right) ;{ }^{1} \mathbf{H}$ NMR $\left(400 \mathrm{~Hz}, \mathrm{CDCl}_{3}\right) \delta 8.05-8.00$ (m, 1H), 7.99-7.89 (m, 3H), 7.80-7.74 (m, 1H), 7.60-7.52 (m, 1H), 7.46-7.36 (m, 2H), 7.26-7.18 (m, 3H), 7.16-7.10 (m, 1H), $4.37(\mathrm{~s}, 1 \mathrm{H}), 4.27(\mathrm{~s}, 1 \mathrm{H}), 4.23(\mathrm{~s}, 5 \mathrm{H})$, 4.20-4.12 (m, 2H), 3.29-2.98 (m, 3H), 2.69-2.59 (m, 1H), $2.18(\mathrm{~s}, 6 \mathrm{H}), 1.27$ (d, $J=6.7$ $\mathrm{Hz}, 3 \mathrm{H}) ;{ }^{13} \mathbf{C} \mathbf{N M R}\left(101 \mathrm{~Hz}, \mathrm{CDCl}_{3}\right) \delta 135.96,134.98,133.75,132.76,132.52$, $132.45,132.28,132.14,128.83,128.37,128.28,128.00,127.88,127.09,126.94$, $126.72,125.89,125.71,124.87,124.62,97.93,77.82,69.66,69.43,68.26,67.99$, 56.92, 39.11, 35.36, 30.30, 8.02; ${ }^{31} \mathbf{P}$ NMR $\left(162 \mathrm{~Hz}, \mathrm{CDCl}_{3}\right) \delta-6.09$; HRMS (ESI) calcd for $\mathrm{C}_{36} \mathrm{H}_{35} \mathrm{FeNP}[\mathrm{M}+\mathrm{H}]^{+}:$568.1857, Found: 568.1844.

$\left(R_{C}, S_{F c}, R_{a x}\right)-7^{\prime}$ was synthesized from $(R)-5(2.94 \mathrm{~g}, 10 \mathrm{mmol})$ and $(R)-2(2.57 \mathrm{~g}, 10$ mmol) according to the same procedure of $\left(R_{C}, S_{F c}, S_{a x}\right)-7$. Characterization of $\left(R_{C}, S_{F c}, R_{a x}\right)-7$ ': yellow foam; $3.69 \mathrm{~g}, 65 \% ;[\boldsymbol{\alpha}]_{\mathbf{D}}^{25}-247.6\left(c=0.25, \mathrm{CHCl}_{3}\right) ;{ }^{1} \mathbf{H}$ NMR $\left(400 \mathrm{~Hz}, \mathrm{CDCl}_{3}\right) \delta 7.98-7.89(\mathrm{~m}, 3 \mathrm{H}), 7.72(\mathrm{t}, J=8.4 \mathrm{~Hz}, 2 \mathrm{H}), 7.44(\mathrm{t}, J=7.2 \mathrm{~Hz}$, 2H), 7.56-7.51 (m, 1H), 7.29-7.20 (m, 3H), $6.78(\mathrm{~d}, J=8.3 \mathrm{~Hz}, 1 \mathrm{H}), 4.34(\mathrm{~s}, 1 \mathrm{H})$, 4.18-4.11 (m, 1H), $4.09(\mathrm{~s}, 1 \mathrm{H}), 4.06(\mathrm{~s}, 5 \mathrm{H}), 3.39(\mathrm{~s}, 1 \mathrm{H}), 2.91-2.74(\mathrm{~m}, 3 \mathrm{H})$, 
2.62-2.53 (m, 1H), $2.18(\mathrm{~s}, 6 \mathrm{H}), 1.29(\mathrm{~d}, J=6.7 \mathrm{~Hz}, 3 \mathrm{H}) ;{ }^{13} \mathbf{C ~ N M R}\left(101 \mathrm{~Hz}, \mathrm{CDCl}_{3}\right)$ $\delta 135.48,135.16,133.81,132.71,132.33,132.21,132.07,131.83,128.93,128.20$, $128.12,127.56,126.96,126.72,126.54,125.79,125.63,124.84,124.46,96.83,73.53$, 70.30, 69.49, 69.26, 67.48, 56.80, 39.17, 32.80, 29.56, 7.89; ${ }^{31} \mathbf{P}$ NMR (162 Hz, $\left.\mathrm{CDCl}_{3}\right) \delta-6.58$; HRMS (ESI) calcd for $\mathrm{C}_{36} \mathrm{H}_{35} \mathrm{FeNP}[\mathrm{M}+\mathrm{H}]^{+}:$568.1857, Found: 568.1839 .

$\left(R_{C}, S_{F c}, S_{a x}\right)$-1. $\left(R_{C}, S_{F c}, S_{a x}\right)-7(567 \mathrm{mg}, 1 \mathrm{mmol})$ was suspended in anhydrous and degassed acetic acid $(5 \mathrm{~mL})$, under an argon atmosphere. After the addition of $\mathrm{R}_{2} \mathrm{PH}$ $\left[(t-\mathrm{Bu})_{2} \mathrm{PH}, 160 \mathrm{mg}, 11 \mathrm{mmol}(\mathrm{a}) ; \mathrm{Cy}_{2} \mathrm{PH}, 218 \mathrm{mg}, 11 \mathrm{mmol}(\mathrm{b}) ; \mathrm{Ph}_{2} \mathrm{PH}, 205 \mathrm{mg}, 11\right.$ mmol (c); (Xyl) $\left.)_{2} \mathrm{PH}, 242 \mathrm{mg}, 11 \mathrm{mmol}(\mathrm{d})\right]$, the mixture was stirred at $95{ }^{\circ} \mathrm{C}$ for $1 \sim 4 \mathrm{~h}$ [1 h (a), $1.5 \mathrm{~h} \mathrm{(b),} 3 \mathrm{~h}$ (c), $4 \mathrm{~h}$ (d)]. The solvent was then evaporated in vucuo and the residue was dissolved in $\mathrm{CH}_{2} \mathrm{Cl}_{2}(20 \mathrm{~mL})$. The organic layer was successively washed with water, saturated $\mathrm{NaHCO}_{3}$ solution and brine, and dried over $\mathrm{Na}_{2} \mathrm{SO}_{4}$. After solvent was evaporated under vacuum, the crude product was purified with chromatography (EtOAc/PE) to afford the desired diphosphine ligands 1 (yield: $65-80 \%)$.

Characterization of $\left(R_{C}, S_{F c}, S_{a x}\right)$-1a: orange power; $434 \mathrm{mg}, 65 \% ;[\mathbf{\alpha}]_{\mathbf{D}}^{25}-200.2(c=$ 0.25, $\left.\mathrm{CHCl}_{3}\right) ;{ }^{1} \mathbf{H}$ NMR $\left(400 \mathrm{~Hz}, \mathrm{CDCl}_{3}\right) \delta$ 8.06-8.01 (m, 1H), 7.98-7.89 (m, 3H), 7.86-7.76 (m, 1H), 7.66-7.56 (m, 1H), 7.46-7.37 (m, 2H), 7.26-7.16 (m, 3H), 7.16-7.10 (m, 1H), 4.36-4.25 (m, 7H), $4.15(\mathrm{~s}, 1 \mathrm{H}), 3.96-3.84(\mathrm{~m}, 1 \mathrm{H}), 3.60-3.50(\mathrm{~m}$, $1 \mathrm{H}), 3.35-3.23(\mathrm{~m}, 1 \mathrm{H}), 3.15-3.00(\mathrm{~m}, 1 \mathrm{H}), 2.63(\mathrm{dd}, J=11.3,4.5 \mathrm{~Hz}, 1 \mathrm{H}), 1.83(\mathrm{dd}$, $J=6.9,3.1 \mathrm{~Hz}, 3 \mathrm{H}), 1.45(\mathrm{~d}, J=10.4 \mathrm{~Hz}, 9 \mathrm{H}), 1.03(\mathrm{~d}, J=10.6 \mathrm{~Hz}, 9 \mathrm{H}) ;{ }^{13} \mathbf{C} \mathbf{N M R}$ $\left(101 \mathrm{~Hz}, \mathrm{CDCl}_{3}\right) \delta 136.48,135.45,133.77,132.51,132.45,132.28,132.18,128.92$, $128.37,128.25,128.02,127.84,127.05,127.00,126.77,125.89$, 125.67, 124.88, $124.58,102.47,70.14,69.43,68.72,67.89,36.54,34.04,31.70,30.75,16.60 ;{ }^{31} \mathbf{P}$ NMR $\left(162 \mathrm{~Hz}, \mathrm{CDCl}_{3}\right) \delta 52.92(\mathrm{~d}, J=44.2 \mathrm{~Hz}),-10.07$ (d, $\left.J=44.4 \mathrm{~Hz}\right)$; HRMS (ESI) calcd for $\mathrm{C}_{42} \mathrm{H}_{47} \mathrm{FeP}_{2}[\mathrm{M}+\mathrm{H}]^{+}$: 669.2502, Found: 669.2493 .

Characterization of $\left(R_{C}, S_{F c}, S_{a x}\right)-\mathbf{1 b}$ : orange foam; $490 \mathrm{mg}, 68 \%$; $[\boldsymbol{\alpha}]_{\mathbf{D}}^{25}-598.4(c=0.25$, 
$\left.\mathrm{CHCl}_{3}\right) ;{ }^{1} \mathbf{H}$ NMR $\left(400 \mathrm{~Hz}, \mathrm{CDCl}_{3}\right) \delta 8.06-8.00(\mathrm{~m}, 1 \mathrm{H}), 7.97-7.88(\mathrm{~m}, 3 \mathrm{H}), 7.83-7.74$ (m, 1H), 7.65-7.58 (m, 1H), 7.48-7.35 (m, 2H), 7.26-7.16 (m, 3H), 7.15-7.07 (m, 1H), 4.31-4.10 (m, 8H), 3.77-3.62 m, 1H), 3.40-3.20 (m, 2H), 3.15-3.00 (m, 1H), 2.67-2.57 $(\mathrm{m}, 1 \mathrm{H}), 1.96-1.63(\mathrm{~m}, 10 \mathrm{H}), 1.59-1.40(\mathrm{~m}, 10 \mathrm{H}), 1.32-1.00(\mathrm{~m}, 5 \mathrm{H}) ;{ }^{13} \mathbf{C}$ NMR $(101$ $\left.\mathrm{Hz}, \mathrm{CDCl}_{3}\right) \delta 136.29,135.07,133.78,132.50,132.40,132.21,128.83,128.35$, $128.23,127.99,127.84,127.06,126.99,126.72$, 125.87, 125.64, 124.89, 124.57, $101.01,69.85,69.30,68.16,67.78,32.92,31.86,31.26,30.05,27.66,27.50,27.07$, 26.70, 26.46, 15.09; ${ }^{31} \mathbf{P}$ NMR (162 MHz, $\left.\mathrm{CDCl}_{3}\right) \delta 17.22(\mathrm{~d}, J=35.4 \mathrm{~Hz}),-10.25(\mathrm{~d}$, $J=35.2 \mathrm{~Hz}$ ); HRMS (ESI) calcd for $\mathrm{C}_{46} \mathrm{H}_{51} \mathrm{FeP}_{2}[\mathrm{M}+\mathrm{H}]^{+}:$721.2815, Found: 721.2808 .

Characterization of $\left(R_{C}, S_{F c}, S_{a x}\right)-1 \mathbf{c}$ : orange solid; $567 \mathrm{mg}, 80 \% ;[\boldsymbol{\alpha}]_{\mathbf{D}}^{25}-698.9(c=0.25$, $\left.\mathrm{CHCl}_{3}\right) ;{ }^{1} \mathbf{H}$ NMR $\left(400 \mathrm{~Hz}, \mathrm{CDCl}_{3}\right) \delta 8.09-8.03(\mathrm{~m}, 1 \mathrm{H}), 8.02-7.95(\mathrm{~m}, 1 \mathrm{H}), 7.93-7.83$ (m, 2H), 7.82-7.75 (m, 1H), 7.51-7.17 (m, 16H), 7.16-7.10 (m, 1H), $4.31(\mathrm{~s}, 5 \mathrm{H}), 4.15$ (s, 2H), 3.96-3.88 (m, 1H), $3.83(\mathrm{~s}, 1 \mathrm{H}), 3.32-3.23(\mathrm{~m}, 1 \mathrm{H}), 3.07$ (t, $J=14.3 \mathrm{~Hz}, 1 \mathrm{H})$, $2.92(\mathrm{t}, J=12.5 \mathrm{~Hz}, 1 \mathrm{H}), 2.54(\mathrm{dd}, J=11.7,4.2 \mathrm{~Hz}, 1 \mathrm{H}), 1.42(\mathrm{t}, J=7.4 \mathrm{~Hz}, 3 \mathrm{H}) ;{ }^{13} \mathrm{C}$ NMR $\left(101 \mathrm{~Hz}, \mathrm{CDCl}_{3}\right) \delta 137.97,137.78,135.85,135.32,135.12,134.66,134.46$, $134.26,133.79,132.60,132.51,132.35,132.25,132.20,132.03,131.88,129.10$, $128.71,128.29,128.03,127.87,127.80,127.55,127.14,127.06,126.69$, 125.91, $125.67,124.98,124.65,98.71,69.80,69.27,68.74,68.09,35.80,30.65,16.41 ;{ }^{31} \mathbf{P}$ NMR $\left(162 \mathrm{~Hz}, \mathrm{CDCl}_{3}\right) \delta 10.98(\mathrm{~d}, J=23.5 \mathrm{~Hz}),-10.82(\mathrm{~d}, J=23.5 \mathrm{~Hz})$; HRMS (ESI) calcd for $\mathrm{C}_{46} \mathrm{H}_{39} \mathrm{FeP}_{2}[\mathrm{M}+\mathrm{H}]^{+}:$709.1876, Found: 709.1874 .

Characterization of $\left(R_{C}, S_{F c}, S_{a x}\right)-\mathbf{1 d}$ : orange solid; $535 \mathrm{mg}, 70 \%$; $[\alpha]_{\mathbf{D}}^{25}-564.5(c=0.25$, $\left.\mathrm{CHCl}_{3}\right)$; ${ }^{1} \mathbf{H}$ NMR $\left(400 \mathrm{~Hz}, \mathrm{CDCl}_{3}\right) \delta$ 8.09-8.03 (m, 1H), 8.02-7.96 (m, $\left.1 \mathrm{H}\right)$, 7.93-7.83 (m, 2H), 7.82-7.76 (m, 1H), 7.54-7.44 (m, 2H), 7.43-7.36 (m, 1H), 7.28-7.18 (m, 3H), 7.16-7.10 (m, 1H), 7.03-6.93 (m, 3H), 6.91-6.82 (m, 3H), $4.30(\mathrm{~s}$, $5 \mathrm{H}), 4.17(\mathrm{~s}, 1 \mathrm{H}), 4.14(\mathrm{~s}, 1 \mathrm{H}), 3.89-3.80(\mathrm{~m}, 1 \mathrm{H}), 3.78(\mathrm{~s}, 1 \mathrm{H}), 3.33-3.23(\mathrm{~m}, 1 \mathrm{H})$, 3.14-3.00 (m, 2H), $2.55(\mathrm{dd}, J=11.7,4.3 \mathrm{~Hz}, 1 \mathrm{H}), 2.29(\mathrm{~s}, 6 \mathrm{H}), 2.24(\mathrm{~s}, 6 \mathrm{H}), 1.40(\mathrm{t}$, $J=7.1 \mathrm{~Hz}, 3 \mathrm{H}) ;{ }^{13} \mathbf{C} \mathbf{N M R}\left(101 \mathrm{~Hz}, \mathrm{CDCl}_{3}\right) \delta 137.78,137.61,137.03,136.95$, $135.95,134.74,134.05,133.82,133.03,132.83,132.53,132.39,132.25,130.73$, 
$129.63,129.49,129.27,128.78,128.26,128.05,127.90,127.24,127.08,126.72$, $125.92,125.68,124.99,124.66,99.00,69.66,69.25,68.48,68.33,35.95,30.84$, 21.47, 21.29, 16.23; ${ }^{31}$ P NMR $\left(162 \mathrm{~Hz}, \mathrm{CDCl}_{3}\right) \delta 10.12(\mathrm{~d}, J=17.5 \mathrm{~Hz}),-10.96(\mathrm{~d}, J$ $=17.2 \mathrm{~Hz}$ ); HRMS (ESI) calcd for $\mathrm{C}_{50} \mathrm{H}_{47} \mathrm{FeP}_{2}[\mathrm{M}+\mathrm{H}]^{+}:$765.2502, Found: 765.2492. $\left(R_{C}, S_{F c}, R_{a x}\right)$-1e was prepared from $\left(R_{C}, S_{F c}, R_{a x}\right)-7^{\prime}(567 \mathrm{mg}, 1 \mathrm{mmol})$ and $(\mathrm{Xyl})_{2} \mathrm{PH}$ (242 $\mathrm{mg}, 11 \mathrm{mmol})$ according to the same synthesis procedure of $\left(R_{C}, S_{F c}, S_{a x}\right)-\mathbf{1 d}$. Characterization of $\left(R_{C}, S_{F c}, R_{a x}\right)$-1e: orange solid; $550 \mathrm{mg}, 72 \%$; $[\alpha]_{\mathrm{D}}^{25}-122.7(c=0.25$, $\left.\mathrm{CHCl}_{3}\right) ;{ }^{1} \mathbf{H}$ NMR $\left(400 \mathrm{~Hz}, \mathrm{CDCl}_{3}\right) \delta 8.02-7.90(\mathrm{~m}, 3 \mathrm{H}), 7.84-7.78(\mathrm{~m}, 1 \mathrm{H})$, 7.76-7.70 (m, 1H), 7.50-7.40 (m, 2H), 7.37-7.32 (m, 1H), 7.29-7.20 (m, 3H), 7.05-6.85 (m, 7H), $4.10(\mathrm{~s}, 6 \mathrm{H}), 3.92(\mathrm{~s}, 1 \mathrm{H}), 3.89-3.80(\mathrm{~m}, 1 \mathrm{H}), 3.30(\mathrm{~s}, 1 \mathrm{H})$, 3.05-2.80 (m, 2H), 2.55-2.40 (m, 2H), $2.34(\mathrm{~s}, 6 \mathrm{H}), 2.27(\mathrm{~s}, 6 \mathrm{H}), 1.47-1.39(\mathrm{~m}, 3 \mathrm{H})$; ${ }^{13}$ C NMR $\left(101 \mathrm{~Hz}, \mathrm{CDCl}_{3}\right) \delta 137.57,137.30,137.07,135.04,134.56,134.37,133.72$, $132.87,132.67,132.48,132.15,131.86,130.74,130.07,129.91,129.55,129.29$, $128.18,127.45,126.85,126.65,125.80,125.65,124.91,124.61,98.47,71.98,69.66$, 69.41, 68.99, 67.99, 32.59, 29.94, 21.40, 21.34, 17.19; ${ }^{31} \mathbf{P}$ NMR $\left(162 \mathrm{~Hz}, \mathrm{CDCl}_{3}\right) \delta$ $11.69(\mathrm{~d}, J=23.5 \mathrm{~Hz}),-10.34(\mathrm{~d}, J=23.5 \mathrm{~Hz})$; HRMS (ESI) calcd for $\mathrm{C}_{50} \mathrm{H}_{47} \mathrm{FeP}_{2}[\mathrm{M}+\mathrm{H}]^{+}:$765.2502, Found: 765.2505.

\section{General procedure for synthesis of $1-\operatorname{aryl}-3,4-\mathrm{DHIQs}{ }^{4,5}$}

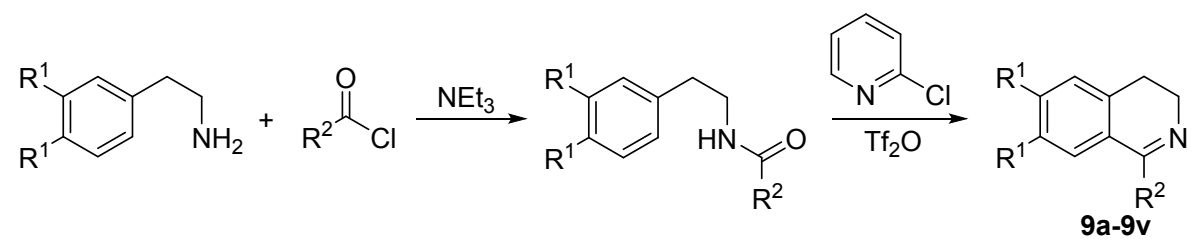

1-aryl-3,4-DHIQs (9a-9v) were synthesized from corresponding 2-arylethyl amine and arylcarbonyl chloride according to reported literature methods. The preparation was performed in air atmosphere. Carbonyl chloride $(10.0 \mathrm{mmol})$ was slowly added to a solution of 2-arylethylamine $(10.0 \mathrm{mmol})$ and triethylamine $(15 \mathrm{mmol})$ in $\mathrm{CH}_{2} \mathrm{Cl}_{2}$ $(60 \mathrm{~mL})$ at $0{ }^{\circ} \mathrm{C}$. After stirring at room temperature for $12 \mathrm{~h}$, the mixture was concentrated and the residue was dissolved in EtOAc $(40 \mathrm{~mL})$. The solution was 
transferred into a separation funnel and washed with saturated $\mathrm{NH}_{4} \mathrm{Cl}$ solution (40 $\mathrm{mL})$. The aqueous layer was further extracted with EtOAc $(2 \times 40 \mathrm{~mL})$. The combined organic layers were washed with brine $(80 \mathrm{~mL})$ and dried over $\mathrm{Na}_{2} \mathrm{SO}_{4}$. Solvent was evaporated to give the $\mathrm{N}$-acyl-2-arylethylamine, which was used in the next step without further purification.

To a solution of the resulting $N$-acyl-2-arylethylamine $(10.0 \mathrm{mmol})$ and 2-chloropyridine (12.0 mmol, 1.2 equiv) in $\mathrm{CH}_{2} \mathrm{Cl}_{2}(60 \mathrm{~mL})$ was slowly added trifluoromethanesulfonic anhydride (11.0 mmol, 1.1 equiv) via syringe dropwise at $-78{ }^{\circ} \mathrm{C}$. Then the mixture was stirred at $-78{ }^{\circ} \mathrm{C}$ for $10 \mathrm{~min}$ and slowly warm to room temperature overnight. The reaction was quenched with saturated $\mathrm{NaHCO}_{3}$ solution and extracted with $\mathrm{CH}_{2} \mathrm{Cl}_{2}(3 \times 50 \mathrm{~mL})$. The combined organic extracts were washed with brine and dried over $\mathrm{Na}_{2} \mathrm{SO}_{4}$. After solvent was evaporated under vacuum, the crude product was purified with chromatography (EtOAc/PE) to yield desired product (yield: 65-95\%).<smiles>c1ccc(C2=NCCc3ccccc32)cc1</smiles>

1-phenyl-3,4-dihydroisoquinoline (9a): ${ }^{5}$ light yellow liquid; 1.86 g, 90\% yield; ${ }^{1} \mathbf{H}$ NMR $\left(400 \mathrm{MHz}, \mathrm{CDCl}_{3}\right) \delta$ 7.68-7.59 (m, 2H), 7.51-7.38 (m, 4H), 7.34-7.24 (m, 3H), 3.94-3.81 (m, 2H), 2.90-2.75 (m, 2H).<smiles>Fc1ccccc1C1=NCCc2ccccc21</smiles>

1-(2-fluorophenyl)-3,4-dihydroisoquinoline (9b): ${ }^{6}$ yellow solid; $1.82 \mathrm{~g}, 81 \%$ yield; ${ }^{1} \mathbf{H}$ NMR (400 MHz, DMSO) $\delta$ 7.68-7.59 (m, 2H), 7.44-7.38 (m, 1H), 7.34-7.21 (m, 4H), $6.92(\mathrm{~d}, J=7.7 \mathrm{~Hz}, 1 \mathrm{H}), 3.82-3.72(\mathrm{~m}, 2 \mathrm{H}), 2.81-2.73(\mathrm{~m}, 2 \mathrm{H})$. 


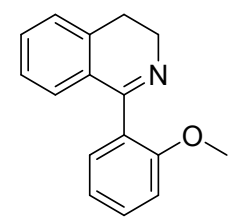

1-(2-methoxyphenyl)-3,4-dihydroisoquinoline (9c): ${ }^{5}$ yellow solid; $1.78 \mathrm{~g} ; 75 \%$ yield; ${ }^{1} \mathbf{H}$ NMR $\left(400 \mathrm{MHz}, \mathrm{CDCl}_{3}\right) \delta 7.42-7.30(\mathrm{~m}, 3 \mathrm{H}), 7.22(\mathrm{~d}, J=7.4 \mathrm{~Hz}, 1 \mathrm{H}), 7.16(\mathrm{t}, J$ $=7.2 \mathrm{~Hz}, 1 \mathrm{H}), 7.07-7.01(\mathrm{~m}, 1 \mathrm{H}), 6.99-6.93(\mathrm{~m}, 2 \mathrm{H}), 4.05-3.75(\mathrm{~m}, 2 \mathrm{H}), 3.68(\mathrm{~s}, 3 \mathrm{H})$, 2.94-2.78 (m, 2H).<smiles>Fc1cccc(C2=NCCc3ccccc32)c1</smiles>

1-(3-fluorophenyl)-3,4-dihydroisoquinoline (9d): ${ }^{7}$ yellow oil; $1.91 \mathrm{~g}, 85 \%$ yield; ${ }^{1} \mathbf{H}$ NMR $\left(400 \mathrm{MHz}, \mathrm{CDCl}_{3}\right) \delta$ 7.47-7.39 (m, 3H), 7.38-7.35 (m, 1H), 7.32-7.25 (m, 3H), 7.20-7.12 (m, 1H), 3.92-3.82 (m, 2H), 2.87-2.77 (m, 2H).<smiles>COc1cccc(C2=NCCc3ccccc32)c1</smiles>

1-(3-methoxyphenyl)-3,4-dihydroisoquinoline (9e): ${ }^{8}$ yellow solid; $1.94 \mathrm{~g}, 82 \%$ yield; ${ }^{1} \mathbf{H}$ NMR $\left(400 \mathrm{MHz}, \mathrm{CDCl}_{3}\right) \delta$ 7.45-7.39 (m, 1H), 7.38-7.25 (m, 4H), 7.21-7.15 (m, 2H), 7.05-7.00 (m, 1H), 3.91-3.85 (m, 2H), $3.87(\mathrm{~s}, 3 \mathrm{H}), 2.87-2.80(\mathrm{~m}, 2 \mathrm{H})$.<smiles>Cc1cccc(C2=NCCc3ccccc32)c1</smiles>

1-(3-methyphenyl)-3,4-dihydroisoquinoline (9f): ${ }^{5}$ yellow oil; 2.03 g, 92\% yield; ${ }^{1} \mathbf{H}$ NMR $\left(400 \mathrm{MHz}, \mathrm{CDCl}_{3}\right) \delta$ 7.50-7.45 (m, 1H), 7.45-7.37 (m, 2H), 7.36-7.24 (m, 5H), 3.93-3.83 (m, 2H), 2.88-2.79 (m, 2H), $2.42(\mathrm{~s}, 3 \mathrm{H})$. 
<smiles>Fc1ccc(C2=NCCc3ccccc32)cc1</smiles>

1-(4-fluorophenyl)-3,4-dihydroisoquinoline (9g): ${ }^{7}$ yellow solid; 1.98 g, 88\% yield; ${ }^{1} \mathbf{H}$ NMR $\left(400 \mathrm{MHz}, \mathrm{CDCl}_{3}\right) \delta$ 7.67-7.58 (m, 2H), 7.46-7.38 (m, 1H), 7.32-7.24 (m, 3H), 7.17-7.09 (m, 2H), 3.90-3.80 (m, 2H), 2.87-2.77 (m, 2H).<smiles>Cc1ccc(C2=NCCc3ccccc32)cc1</smiles>

1-(4-methyphenyl)-3,4-dihydroisoquinoline (9h): ${ }^{5}$ white solid; $2.1 \mathrm{~g}, 95 \%$ yield; ${ }^{1} \mathbf{H}$ NMR $\left(400 \mathrm{MHz}, \mathrm{CDCl}_{3}\right) \delta$ 7.56-7.44 (m, 2H), 7.42-7.34 (m, 1H), 7.29-7.16 (m, 5H), 3.88-3.78 (m, 2H), 2.85-2.72 (m, 2H), $2.41(\mathrm{~s}, 3 \mathrm{H})$.<smiles>FC(F)(F)c1ccc(C2=NCCc3ccccc32)cc1</smiles>

1-(4-(trifluoromethyl)phenyl)-3,4-dihydroisoquinoline (9i): ${ }^{5}$ white solid; $2.53 \mathrm{~g}, 92 \%$ yield; ${ }^{1}$ H NMR (400 MHz, $\mathrm{CDCl}_{3}$ ) $\delta$ 7.82-7.61 (m, 4H), 7.46-7.36 (m, 1H), 7.33-7.23 (m, 2H), 7.22-7.15 (m, 1H), 3.98-3.79 (m, 2H), $2.82(\mathrm{t}, J=7.4 \mathrm{~Hz}, 2 \mathrm{H})$.<smiles></smiles>

6,7-dimethoxy-1-phenyl-3,4-dihydroisoquinoline (9j): ${ }^{5}$ white solid; $2.40 \mathrm{~g}, 90 \%$ yield; ${ }^{1} \mathbf{H}$ NMR $\left(400 \mathrm{MHz}, \mathrm{CDCl}_{3}\right) \delta$ 7.67-7.58 (m, 2H), 7.50-7.40 (m, 3H), $6.81(\mathrm{~d}, J$ $=4.8 \mathrm{~Hz}, 2 \mathrm{H}), 3.97(\mathrm{~s}, 3 \mathrm{H}), 3.84(\mathrm{t}, J=7.5 \mathrm{~Hz}, 2 \mathrm{H}), 3.75(\mathrm{~s}, 3 \mathrm{H}), 2.76(\mathrm{t}, J=7.4 \mathrm{~Hz}$, 
$2 \mathrm{H})$.<smiles>COc1cc2c(cc1OC)C(c1ccccc1F)=NCC2</smiles>

1-(2-fluorophenyl)-6,7-dimethoxy-3,4-dihydroisoquinoline (9k): ${ }^{9}$ yellow oil; $2.28 \mathrm{~g}$, $80 \%$ yield; ${ }^{1} \mathbf{H}$ NMR (400 MHz, DMSO) $\delta$ 7.57-7.42 (m, 2H), 7.36-7.25 (m, 2H), $6.98(\mathrm{~s}, 1 \mathrm{H}), 6.45(\mathrm{~s}, 1 \mathrm{H}), 3.83(\mathrm{~s}, 3 \mathrm{H}), 3.73(\mathrm{t}, J=7.7 \mathrm{~Hz}, 2 \mathrm{H}), 3.54(\mathrm{~s}, 3 \mathrm{H}), 2.71(\mathrm{t}, J$ $=7.7 \mathrm{~Hz}, 2 \mathrm{H})$.<smiles>COc1cc2c(cc1OC)C(c1ccccc1OC)=NCC2</smiles>

6,7-dimethoxy-1-(2-methoxyphenyl)-3,4-dihydroisoquinoline (91): ${ }^{9}$ yellow solid; 1.96 g, 66\% yield; ${ }^{1} \mathbf{H}$ NMR (400 MHz, $\left.\mathrm{CDCl}_{3}\right) \delta 7.45-7.35(\mathrm{~m}, 2 \mathrm{H}), 7.09-7.03(\mathrm{~m}, 1 \mathrm{H})$, $6.97(\mathrm{~d}, J=8.2 \mathrm{~Hz}, 1 \mathrm{H}), 6.76(\mathrm{~s}, 1 \mathrm{H}), 6.54(\mathrm{~s}, 1 \mathrm{H}), 4.18-3.80(\mathrm{~m}, 2 \mathrm{H}), 3.95(\mathrm{~s}, 3 \mathrm{H})$, $3.72(\mathrm{~s}, 3 \mathrm{H}), 3.67(\mathrm{~s}, 3 \mathrm{H}), 2.81(\mathrm{~s}, 2 \mathrm{H})$.<smiles>COc1cc2c(cc1OC)C(c1ccccc1C)=NCC2</smiles>

6,7-dimethoxy-1-(2-methyphenyl)-3,4-dihydroisoquinoline (9m): ${ }^{9}$ light yellow oil; $1.97 \mathrm{~g}, 70 \%$ yield; ${ }^{1} \mathbf{H}$ NMR $\left(400 \mathrm{MHz}, \mathrm{CDCl}_{3}\right) \delta$ 7.35-7.22 (m, 4H), $6.78(\mathrm{~s}, 1 \mathrm{H})$, $6.45(\mathrm{~s}, 1 \mathrm{H}), 3.95(\mathrm{~s}, 3 \mathrm{H}), 3.98-3.76(\mathrm{~m}, 2 \mathrm{H}), 3.65(\mathrm{~s}, 3 \mathrm{H}), 2.81(\mathrm{t}, J=7.6 \mathrm{~Hz}, 2 \mathrm{H})$, $2.16(\mathrm{~s}, 3 \mathrm{H})$.<smiles>COc1cc2c(cc1OC)C(c1cccc(F)c1)=NCC2</smiles>

1-(3-fluorophenyl)-6,7-dimethoxy-3,4-dihydroisoquinoline (9n): ${ }^{10}$ white solid; $2.51 \mathrm{~g}$, 
88\% yield; ${ }^{1} \mathbf{H}$ NMR $\left(400 \mathrm{MHz}, \mathrm{CDCl}_{3}\right) \delta$ 7.46-7.33 (m, 3H), 7.21-7.12 (m, 1H), 6.80 (d, $J=9.7 \mathrm{~Hz}, 2 \mathrm{H}), 3.97(\mathrm{~s}, 3 \mathrm{H}), 3.84(\mathrm{t}, J=7.6 \mathrm{~Hz}, 2 \mathrm{H}), 3.76(\mathrm{~s}, 3 \mathrm{H}), 2.80-2.72(\mathrm{~m}$, $2 \mathrm{H})$.<smiles>COc1cccc(C2=NCCc3cc(OC)c(OC)cc32)c1</smiles>

6,7-dimethoxy-1-(3-methoxyphenyl)-3,4-dihydroisoquinoline (9o): ${ }^{9}$ white solid; 2.23 g, 75\% yield; ${ }^{1} \mathbf{H}$ NMR $\left(400 \mathrm{MHz}, \mathrm{CDCl}_{3}\right) \delta 7.35$ (t, $\left.J=7.9 \mathrm{~Hz}, 1 \mathrm{H}\right), 7.22-7.16(\mathrm{~m}$, 2H), 7.04-6.99 (m, 1H), $6.85(\mathrm{~s}, 1 \mathrm{H}), 6.80(\mathrm{~s}, 1 \mathrm{H}), 3.97(\mathrm{~s}, 3 \mathrm{H}), 3.87(\mathrm{~s}, 3 \mathrm{H})$, 3.86-3.81 (m, 2H), $3.76(\mathrm{~s}, 3 \mathrm{H}), 2.80-2.72(\mathrm{~m}, 2 \mathrm{H})$.<smiles>COc1cc2c(cc1OC)C(c1cccc(C)c1)=NCC2</smiles>

6,7-dimethoxy-1-(3-methyphenyl)-3,4-dihydroisoquinoline (9p): ${ }^{9}$ yellow oil; $2.53 \mathrm{~g}$, 90\% yield; ${ }^{1} \mathbf{H}$ NMR $\left(400 \mathrm{MHz}, \mathrm{CDCl}_{3}\right) \delta 7.50(\mathrm{~s}, 1 \mathrm{H}), 7.42-7.37$ (m, 1H), $7.33(\mathrm{t}, J=$ $7.4 \mathrm{~Hz}, 1 \mathrm{H}), 7.30-7.26(\mathrm{~m}, 1 \mathrm{H}), 6.84(\mathrm{~s}, 1 \mathrm{H}), 6.81(\mathrm{~s}, 1 \mathrm{H}), 3.98(\mathrm{~s}, 3 \mathrm{H}), 3.87-3.80$ (m, $2 \mathrm{H}), 3.75(\mathrm{~s}, 3 \mathrm{H}), 2.80-2.72(\mathrm{~m}, 2 \mathrm{H}), 2.42(\mathrm{~s}, 3 \mathrm{H})$.<smiles>COc1cc2c(cc1OC)C(c1ccc(F)cc1)=NCC2</smiles>

1-(4-fluorophenyl)-6,7-dimethoxy-3,4-dihydroisoquinoline (9q): ${ }^{9}$ white solid; $2.65 \mathrm{~g}$, 93\% yield; ${ }^{1} \mathbf{H}$ NMR (400 MHz, DMSO) $\delta$ 7.67-7.55 (m, 2H), $7.28(\mathrm{t}, J=8.5 \mathrm{~Hz}$, 2H), $7.01(\mathrm{~s}, 1 \mathrm{H}), 6.69(\mathrm{~s}, 1 \mathrm{H}), 3.85(\mathrm{~s}, 3 \mathrm{H}), 3.66(\mathrm{t}, J=7.4 \mathrm{~Hz}, 2 \mathrm{H}), 3.62(\mathrm{~s}, 3 \mathrm{H})$, $2.66(\mathrm{t}, J=7.4 \mathrm{~Hz}, 2 \mathrm{H})$. 
<smiles>COc1cc2c(cc1OC)C(c1ccc(Cl)cc1)=NCC2</smiles>

1-(4-chlorophenyl)-6,7-dimethoxy-3,4-dihydroisoquinoline (9r): ${ }^{7}$ white solid; $2.71 \mathrm{~g}$, 90\% yield; ${ }^{1}$ H NMR (400 MHz, DMSO) $\delta 7.56(\mathrm{~d}, J=8.4 \mathrm{~Hz}, 2 \mathrm{H}), 7.41(\mathrm{~d}, J=8.4$ Hz, 2H), $6.79(\mathrm{~s}, 1 \mathrm{H}), 6.74(\mathrm{~s}, 1 \mathrm{H}), 3.95(\mathrm{~s}, 3 \mathrm{H}), 3.86-3.77(\mathrm{~m}, 2 \mathrm{H}), 3.74(\mathrm{~s}, 3 \mathrm{H})$, 2.80-2.66 (m, 2H).<smiles>COc1ccc(C2=NCCc3cc(OC)c(OC)cc32)cc1</smiles>

6,7-dimethoxy-1-(4-methoxyphenyl)-3,4-dihydroisoquinoline (9s): ${ }^{9}$ white solid; 2.38 g, 90\% yield; ${ }^{1} \mathbf{H}$ NMR $\left(400 \mathrm{MHz}, \mathrm{CDCl}_{3}\right) \delta$ 7.63-7.56 (m, 2H), 7.00-6.93 (m, 2H), $6.86(\mathrm{~s}, 1 \mathrm{H}), 6.80(\mathrm{~s}, 1 \mathrm{H}), 3.97(\mathrm{~s}, 3 \mathrm{H}), 3.88(\mathrm{~s}, 3 \mathrm{H}), 3.82-3.77(\mathrm{~m}, 2 \mathrm{H}), 3.77(\mathrm{~s}, 3 \mathrm{H})$, 2.79-2.69 (m, 2H).<smiles>COc1cc2c(cc1OC)C(c1ccc(C)cc1)=NCC2</smiles>

6,7-dimethoxy-1-(4-methyphenyl)-3,4-dihydroisoquinoline $(9 \mathrm{t}):^{8}$ yellow solid; $1.83 \mathrm{~g}$, 65\% yield; ${ }^{1} \mathbf{H}$ NMR (400 MHz, DMSO) $\delta$ 7.50-7.42 (m, 2H), 7.29-7.21 (m, 2H), $6.99(\mathrm{~s}, 1 \mathrm{H}), 6.72(\mathrm{~s}, 1 \mathrm{H}), 3.84(\mathrm{~s}, 3 \mathrm{H}), 3.67-3.62(\mathrm{~m}, 2 \mathrm{H}), 3.60(\mathrm{~s}, 3 \mathrm{H}), 2.68-2.60(\mathrm{~m}$, 2H), 2.37 (s, 3H).<smiles>COc1ccc(C2=NCCc3cc(OC)c(OC)cc32)cc1</smiles> 
1-(3,4-dimethoxyphenyl)-6,7-dimethoxy-3,4-dihydroisoquinoline $(9 \mathbf{9 u}):^{5}$ white solid; 3.04 g, 93\% yield; ${ }^{1} \mathbf{H}$ NMR (400 MHz, DMSO) $\delta 7.24$ (s, 1H), 7.16 (d, $J=8.4 \mathrm{~Hz}$, 1H), 6.96-6.85 (m, 2H), 6.79 (d, J=2.1 Hz, 1H), 3.95 (s, 3H), $3.94(\mathrm{~s}, 3 \mathrm{H}), 3.92$ (s, $3 \mathrm{H}), 3.82-3.77(\mathrm{~m}, 2 \mathrm{H}), 3.76(\mathrm{~s}, 3 \mathrm{H}), 2.72(\mathrm{t}, J=7.6 \mathrm{~Hz}, 1 \mathrm{H})$.<smiles>COc1cc2c(cc1OC)C(c1cc(OC)c(OC)c(OC)c1)=NCC2</smiles>

6,7-dimethoxy-1-(3,4,5-trimethoxyphenyl)-3,4-dihydroisoquinoline (9v): ${ }^{5}$ white solid; $3.28 \mathrm{~g}, 92 \%$ yield; ${ }^{1} \mathrm{H}$ NMR (400 MHz, DMSO) $\delta 6.88(\mathrm{~s}, 1 \mathrm{H}), 6.86$ (s, 2H), 6.80 (s, 1H), $3.96(\mathrm{~s}, 3 \mathrm{H}), 3.90(\mathrm{~s}, 3 \mathrm{H}), 3.87(\mathrm{~s}, 6 \mathrm{H}), 3.84-3.78(\mathrm{~m}, 2 \mathrm{H}), 3.77(\mathrm{~s}, 3 \mathrm{H}), 2.73(\mathrm{t}, J$ $=7.4 \mathrm{~Hz}, 2 \mathrm{H})$.<smiles>COc1cc2c(cc1OC)C(C)=NCC2</smiles>

6,7-dimethoxy-1-methyl-3,4-dihydroisoquinoline $(9 \mathbf{9 w}):^{11}$ yellow oil; $1.44 \mathrm{~g}, 70 \%$ yield; ${ }^{1} \mathbf{H}$ NMR $\left(400 \mathrm{MHz}, \mathrm{CDCl}_{3}\right) \delta 7.00(\mathrm{~s}, 1 \mathrm{H}), 6.70(\mathrm{~s}, 1 \mathrm{H}), 3.93(\mathrm{~d}, J=3.5 \mathrm{~Hz}$, $6 \mathrm{H}), 3.69-3.60(\mathrm{~m}, 2 \mathrm{H}), 2.70-2.60(\mathrm{~m}, 2 \mathrm{H}), 2.38(\mathrm{~s}, 3 \mathrm{H})$.<smiles>CCC1=NCCc2cc(OC)c(OC)cc21</smiles>

6,7-dimethoxy-1- ethyl-3,4-dihydroisoquinoline $(\mathbf{9 x}):{ }^{11}$ yellow oil; $1.71 \mathrm{~g}, 78 \%$ yield; ${ }^{1} \mathbf{H}$ NMR $\left(400 \mathrm{MHz}, \mathrm{CDCl}_{3}\right) \delta 7.00(\mathrm{~s}, 1 \mathrm{H}), 6.69(\mathrm{~s}, 1 \mathrm{H}), 3.90(\mathrm{~d}, J=4.6 \mathrm{~Hz}, 6 \mathrm{H})$, 3.73-3.37 (m, 2H), 2.79-2.67 (m, 2H), 2.68-2.53 (m, 2H), $1.22(\mathrm{t}, J=7.4 \mathrm{~Hz}, 3 \mathrm{H})$. 


\section{Optimization of $\mathrm{AH}$ reaction conditions}

Table S1: Optimization of reaction conditions for the $\mathrm{AH}$ of $9 \mathbf{a}^{a}$

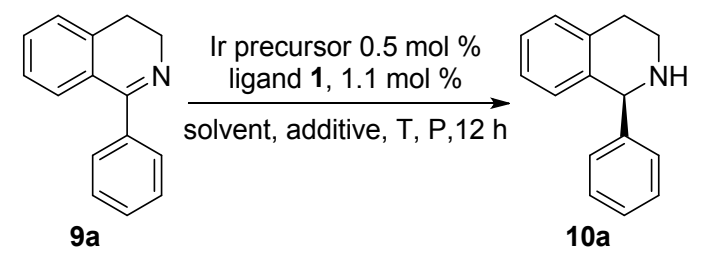

\begin{tabular}{|c|c|c|c|c|c|c|c|}
\hline $\begin{array}{l}\text { Entr } \\
\mathrm{y}\end{array}$ & Ir precursor & Ligand & Solvent & Additive & $\begin{array}{c}\mathrm{T} \\
\left({ }^{\circ} \mathrm{C}\right) / \\
\mathrm{P} \\
(\mathrm{atm})\end{array}$ & $\begin{array}{r}\text { Con } \\
\text { V. } \\
b \%\end{array}$ & $\begin{array}{l}e e^{c} \\
\%\end{array}$ \\
\hline 1 & {$[\operatorname{Ir}(\mathrm{COD}) \mathrm{Cl}]_{2}$} & $\left(R_{C}, S_{F c}, S_{a x}\right)-\mathbf{1 a}$ & toluene & $\mathrm{I}_{2}, \mathrm{TFA}$ & $30 / 50$ & $>99$ & 81 \\
\hline 2 & {$[\operatorname{Ir}(\mathrm{COD}) \mathrm{Cl}]_{2}$} & $\left(R_{C}, S_{F c}, S_{a x}\right)-\mathbf{1 b}$ & toluene & $\mathrm{I}_{2}, \mathrm{TFA}$ & $30 / 50$ & $>99$ & 60 \\
\hline 3 & {$[\operatorname{Ir}(\mathrm{COD}) \mathrm{Cl}]_{2}$} & $\left(R_{C}, S_{F c}, S_{a x}\right)-\mathbf{1 c}$ & toluene & $\mathrm{I}_{2}, \mathrm{TFA}$ & $30 / 50$ & $>99$ & 22 \\
\hline 4 & {$[\operatorname{Ir}(\mathrm{COD}) \mathrm{Cl}]_{2}$} & $\left(R_{C}, S_{F c}, S_{a x}\right)-\mathbf{1 d}$ & toluene & $\mathrm{I}_{2}, \mathrm{TFA}$ & $30 / 50$ & $>99$ & 65 \\
\hline 5 & {$[\operatorname{Ir}(\mathrm{COD}) \mathrm{Cl}]_{2}$} & $\left(R_{C}, S_{F c}, R_{a x}\right)-\mathbf{1 e}$ & toluene & $\mathrm{I}_{2}, \mathrm{TFA}$ & $30 / 50$ & $>99$ & 15 \\
\hline $6^{\mathrm{d}}$ & {$\left[\operatorname{Ir}(\mathrm{COD})_{2}\right] \mathrm{BF}_{4}$} & $\left(R_{C}, S_{F c}, S_{a x}\right)-\mathbf{1 a}$ & toluene & $\mathrm{I}_{2}, \mathrm{TFA}$ & $30 / 50$ & $>99$ & 79 \\
\hline
\end{tabular}


$7 \quad\left[\operatorname{Ir}(\mathrm{COD})_{2}\right] \mathrm{BArF} \quad\left(R_{C}, S_{F c}, S_{a x}\right)-\mathbf{1 a} \quad$ toluene

$8 \quad[\operatorname{Ir}(\mathrm{COD}) \mathrm{Cl}]_{2} \quad\left(R_{C}, S_{F c}, S_{a x}\right)-\mathbf{1 a} \quad \mathrm{CHCl}_{3}$

$\mathrm{I}_{2}$, TFA $\quad 30 / 50 \quad>99 \quad 80$

$[\operatorname{Ir}(\mathrm{COD}) \mathrm{Cl}]_{2}$

$\left(R_{C}, S_{F c}, S_{a x}\right)-\mathbf{1 a} \quad \mathrm{CH}_{2} \mathrm{Cl}_{2}$

$\mathrm{I}_{2}$, TFA $\quad 30 / 50 \quad 98$

68

$10 \quad[\operatorname{Ir}(\mathrm{COD}) \mathrm{Cl}]_{2} \quad\left(R_{C}, S_{F c}, S_{a x}\right)-\mathbf{1 a} \quad 1,4$

$1,4-$

$\mathrm{I}_{2}$, TFA $\quad 30 / 50 \quad 96 \quad 56$

$11 \quad[\operatorname{Ir}(\mathrm{COD}) \mathrm{Cl}]_{2} \quad\left(R_{C}, S_{F c}, S_{a x}\right)-\mathbf{1 a} \quad \mathrm{THF}$

$\mathrm{I}_{2}$, TFA $\quad 30 / 50 \quad>99 \quad 32$

$12 \quad[\operatorname{Ir}(\mathrm{COD}) \mathrm{Cl}]_{2} \quad\left(R_{C}, S_{F c}, S_{a x}\right)-\mathbf{1 a} \quad \mathrm{TBM}$

$\mathrm{I}_{2}, \mathrm{TFA} \quad 30 / 50 \quad>99 \quad 84$

$13 \quad[\mathrm{Ir}(\mathrm{COD}) \mathrm{Cl}]_{2}$

$\left(R_{C}, S_{F c}, S_{a x}\right)-\mathbf{1 a} \quad$ EtOAc

$\mathrm{I}_{2}$, TFA

$30 / 50 \quad 55$

33

$14 \quad[\operatorname{Ir}(\mathrm{COD}) \mathrm{Cl}]_{2} \quad\left(R_{C}, S_{F c}, S_{a x}\right)-\mathbf{1 a} \quad \mathrm{TH}$

$\mathrm{I}_{2}$, TFA $\quad 30 / 50 \quad>99 \quad 55$

15

$[\operatorname{Ir}(\mathrm{COD}) \mathrm{Cl}]_{2}$

$\left(R_{C}, S_{F c}, S_{a x}\right)-\mathbf{1 a} \quad \mathrm{THF}$

none

$30 / 50>99$

74

16

$[\operatorname{Ir}(\mathrm{COD}) \mathrm{Cl}]_{2}$

$\left(R_{C}, S_{F c}, S_{a x}\right)-\mathbf{1 a} \quad \mathrm{THF}$

$\mathrm{I}_{2}$

$30 / 50>99 \quad 75$

17

$[\operatorname{Ir}(\mathrm{COD}) \mathrm{Cl}]_{2}$

$\left(R_{C}, S_{F c}, S_{a x}\right)-\mathbf{1 a} \quad$ THF

TFA

$30 / 50>99$

85

$\mathrm{CH}_{3} \mathrm{COOH} \quad 30 / 50>99 \quad 80$

$18 \quad[\operatorname{Ir}(\mathrm{COD}) \mathrm{Cl}]_{2} \quad\left(R_{C}, S_{F c}, S_{a x}\right)-\mathbf{1 a} \quad$ THF

$\mathrm{HCOOH} 30 / 50>99 \quad 85$

19

$[\mathrm{Ir}(\mathrm{COD}) \mathrm{Cl}]_{2}$

$\left(R_{C}, S_{F c}, S_{a x}\right)-\mathbf{1 a} \quad$ THF

$\mathrm{TsOH}$

$30 / 50>99$

82

20

21

$[\operatorname{Ir}(\mathrm{COD}) \mathrm{Cl}]_{2}$

$\left(R_{C}, S_{F c}, S_{a x}\right)-\mathbf{1 a} \quad$ THF

$\mathrm{PhCOOH}$

$30 / 50>99$

$[\operatorname{Ir}(\mathrm{COD}) \mathrm{Cl}]_{2}$

$\left(R_{C}, S_{F c}, S_{a x}\right)-\mathbf{1 a}$

THF

$98 \% \mathrm{H}_{2} \mathrm{SO}_{4} \quad 30 / 50>99$

86

22

23

$[\operatorname{Ir}(\mathrm{COD}) \mathrm{Cl}]_{2}$

$\left(R_{C}, S_{F c}, S_{a x}\right)-\mathbf{1 a} \quad \mathrm{THF}$

$36 \% \mathrm{HCl}$

$30 / 50>99$

97

$23 \quad[\operatorname{Ir}(\mathrm{COD}) \mathrm{Cl}]_{2} \quad\left(R_{C}, S_{F c}, S_{a x}\right)-\mathbf{1 a} \quad$ THF

$40 \% \mathrm{HBr} \quad 30 / 50>99>99$

24

25

26

$[\operatorname{Ir}(\mathrm{COD}) \mathrm{Cl}]_{2}$

$\left(R_{C}, S_{F c}, S_{a x}\right)-\mathbf{1 a}$

THF

$57 \% \mathrm{HI}$

$30 / 50>99 \quad 58$

$25^{\mathrm{e}} \quad[\operatorname{Ir}(\mathrm{COD}) \mathrm{Cl}]_{2}$

$\left(R_{C}, S_{F c}, S_{a x}\right)-\mathbf{1 a} \quad$ THF

$40 \% \mathrm{HBr}$

$30 / 50>99$

93

$26 \quad[\operatorname{Ir}(\mathrm{COD}) \mathrm{Cl}]_{2} \quad\left(R_{C}, S_{F c}, S_{a x}\right)-\mathbf{1 a} \quad \mathrm{TH}$

$40 \% \mathrm{HBr} \quad 30 / 30 \quad 75 \quad 99$

$27^{\mathrm{f}} \quad[\operatorname{Ir}(\mathrm{COD}) \mathrm{Cl}]_{2} \quad\left(R_{C}, S_{F c}, S_{a x}\right)-\mathbf{1 a} \quad$ THF

$40 \% \mathrm{HBr} \quad 30 / 30 \quad 85 \quad 98$

$28^{\mathrm{g}} \quad[\operatorname{Ir}(\mathrm{COD}) \mathrm{Cl}]_{2} \quad\left(R_{C}, S_{F c}, S_{a x}\right)-\mathbf{1 a} \quad \mathrm{THF}$

$40 \% \mathrm{HBr} \quad 30 / 50 \quad 89 \quad 99$

29, $\mathrm{g}, \quad[\operatorname{Ir}(\mathrm{COD}) \mathrm{Cl}]_{2} \quad\left(R_{C}, S_{F c}, S_{a x}\right)-\mathbf{1 a} \quad \mathrm{THF}$

$40 \% \mathrm{HBr} \quad 30 / 50 \quad 90 \quad 99$

$30^{\mathrm{g}} \quad[\operatorname{Ir}(\mathrm{COD}) \mathrm{Cl}]_{2} \quad\left(R_{C}, S_{F c}, S_{a x}\right)-\mathbf{1 a} \quad \mathrm{THF}$

$40 \% \mathrm{HBr} \quad 50 / 50 \quad 98 \quad 99$

$31^{\mathrm{h}} \quad[\operatorname{Ir}(\mathrm{COD}) \mathrm{Cl}]_{2} \quad\left(R_{C}, S_{F c}, S_{a x}\right)-\mathbf{1 a} \quad \mathrm{THF} \quad 40 \% \mathrm{HBr} \quad 50 / 50 \quad 65 \quad 98$

$32^{\mathrm{h}, \mathrm{f}} \quad[\operatorname{Ir}(\mathrm{COD}) \mathrm{Cl}]_{2} \quad\left(R_{C}, S_{F c}, S_{a x}\right)-\mathbf{1 a} \quad \mathrm{THF} \quad 40 \% \mathrm{HBr} \quad 50 / 50 \quad 85 \quad 98$

${ }^{a}$ Reaction conditions : Ir/ligand/additive/substrate $=1: 2.2: 20: 200,($ substrate $)=0.25 \mathrm{M}$, $50 \mathrm{~atm} \mathrm{H}_{2}, 12$ h. ${ }^{b}$ Conversions were determined by ${ }^{1} \mathrm{H}$ NMR. ${ }^{c}$ Eantiomeric excesses were determined by chiral HPLC after the products were converted into the corresponding 
acetamides. ${ }^{d}$ Ir precursor amount is $1 \mathrm{~mol} \%$. ${ }^{e}$ Additive amount is $1 \mathrm{~mol} \% .{ }^{f}$ Reaction time is $24 \mathrm{~h} .{ }^{g}$ Catalyst loading is $0.1 \mathrm{~mol} \mathrm{\%}$. ${ }^{h}$ Catalyst loading is $0.02 \mathrm{~mol} \%$.

\section{General procedure for $\mathrm{AH}$ and scaled-up catalytic hydrogenation}

To a hydrogenation vessel was added the catalyst precursor $[\operatorname{Ir}(\mathrm{COD}) \mathrm{Cl}]_{2}(1.6 \mathrm{mg}, 2.4$ $\mu \mathrm{mol})$, ligand $\left(R_{C}, S_{F c}, S_{a x}\right)$-1a $(3.6 \mathrm{mg}, 5.4 \mu \mathrm{mol})$ and THF $(1 \mathrm{~mL})$ under nitrogen atmosphere. The mixture was stirred for $20 \mathrm{~min}$ at room temperature to give a homogeneous solution. Then imine substrates $9(0.5 \mathrm{mmol})$ and $40 \% \mathrm{HBr}(7 \mu \mathrm{L}, 0.05$ mmol, $10 \mathrm{~mol} \%$ ) were added and the total solution was made to $2.0 \mathrm{~mL}$. The resulting vessel was transferred to an autoclave, which was replaced with $50 \mathrm{~atm}$ of $\mathrm{H}_{2}$ for 3 times, and the reaction mixtures were stirred at room temperature for $12 \mathrm{~h}$. The hydrogen gas was released slowly and the solution was concentrated. The residue was dissolved in EtOAc $(20 \mathrm{~mL})$, washed with saturated $\mathrm{NaHCO}_{3}$ solution, brine and dried over $\mathrm{Na}_{2} \mathrm{SO}_{4}$. The solvent was evaporated to afford the crude product, which was analyzed by ${ }^{1} \mathrm{H}$ NMR to determine the conversions. The resulting product reacted with acetic anhydride to yield the corresponding acetamide, which was analyzed by HPLC on a chiral stationary phase to determine the enantiomeric excesses.<smiles>c1ccc(C2NCCc3ccccc32)cc1</smiles>

(S)-1-phenyl-1,2,3,4-tetrahydroisoquinoline (10a): ${ }^{5}$ white solid; $103.5 \mathrm{mg}, 99 \%$ yield; ${ }^{1} \mathbf{H}$ NMR $\left(400 \mathrm{MHz}, \mathrm{CDCl}_{3}\right) \delta$ 7.35-7.24 (m, 5H), 7.17-7.12 (m, 2H), 7.07-7.00 (m, $1 \mathrm{H}), 6.75(\mathrm{~d}, J=7.7 \mathrm{~Hz}, 1 \mathrm{H}), 5.11(\mathrm{~s}, 1 \mathrm{H}), 3.33-3.22(\mathrm{~m}, 1 \mathrm{H}), 3.15-2.99(\mathrm{~m}, 2 \mathrm{H})$, 2.88-2.77 (m, 1H), $1.99(\mathrm{~s}, 1 \mathrm{H})$; Enantiomeric excess was determined by HPLC for the corresponding acetamide, Chiralpak OD-H + OD-H column, Hex/IPA = 96.5:3.5, $1 \mathrm{~mL} / \mathrm{min}$. 


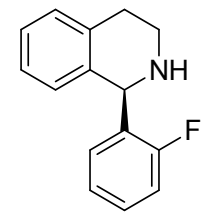

(R)-1-(2-fluorophenyl)-1,2,3,4-tetrahydroisoquinoline (10b): white solid; $112.2 \mathrm{mg}$, 98\% yield; ${ }^{1} \mathbf{H}$ NMR $\left(400 \mathrm{MHz}, \mathrm{CDCl}_{3}\right) \delta$ 7.30-7.20 (m, 1H), 7.18-7.12 (m, 2H), 7.11-7.00 (m, 4H), 6.82-6.74 (m, 1H), $5.51(\mathrm{~s}, 1 \mathrm{H}), 3.27-3.17(\mathrm{~m}, 1 \mathrm{H}), 3.14-3.05(\mathrm{~m}$, 1H), 3.04-2.93 (m, 1H), 2.90-2.82 (m, 1H), 2.02 (s, 1H); ${ }^{13} \mathbf{C}$ NMR (101 MHz, $\left.\mathrm{CDCl}_{3}\right) \delta 162.34,159.89,137.07,135.70,131.74,131.60,130.83,129.15,128.90$, 127.72, 126.43, 125.78, 123.97, 115.58, 115.35, 54.70, 41.77, 29.64; HRMS (ESI) calcd for $\mathrm{C}_{15} \mathrm{H}_{15} \mathrm{FN}[\mathrm{M}+\mathrm{H}]^{+}$: 228.1189, Found: 228.1184; Enantiomeric excess was determined by HPLC for the corresponding acetamide, Chiralpak AD-H column, $\mathrm{Hex} / \mathrm{IPA}=95: 5,1 \mathrm{~mL} / \mathrm{min}$.<smiles>COc1ccccc1C1NCCc2ccccc21</smiles>

(R)-1-(2-methoxyphenyl)-1,2,3,4-tetrahydroisoquinoline (10c): ${ }^{5}$ yellow oil; $115.9 \mathrm{mg}$, 97\% yield; ${ }^{1} \mathbf{H}$ NMR $\left(400 \mathrm{MHz}, \mathrm{CDCl}_{3}\right) \delta$ 7.24-7.20 (m, 1H), 7.17-7.12 (m, 2H), 7.08-7.02 (m, 1H), 6.94-6.92 (m, 1H), 6.86-6.76 (m, 3H), $5.55(\mathrm{~s}, 1 \mathrm{H}), 3.86(\mathrm{~s}, 3 \mathrm{H})$, 3.16-3.08 (m, 1H), 3.06-2.98 (m, 1H), 2.94-2.86 (m, 2H), $2.31(\mathrm{~s}, 1 \mathrm{H})$; Enantiomeric excess was determined by HPLC for the corresponding acetamide, Chiralpak OD-H column, Hex/IPA = 95:5, $1 \mathrm{~mL} / \mathrm{min}$.<smiles>Fc1cccc(C2NCCc3ccccc32)c1</smiles>

(S)-1-(3-fluorophenyl)-1,2,3,4-tetrahydroisoquinoline (10d): ${ }^{7}$ white solid; $112.2 \mathrm{mg}$, 98\% yield; ${ }^{1} \mathbf{H}$ NMR (400 MHz, $\left.\mathrm{CDCl}_{3}\right) \delta$ 7.32-7.26 (m, 1H), 7.32-7.26 (m, 2H), 7.19-7.12 (m, 2H), 7.01-6.91 (m, 2H), 6.78-6.72 (m, 1H), $5.10(\mathrm{~s}, 1 \mathrm{H}), 3.30-3.20(\mathrm{~m}$, $1 \mathrm{H}), 3.14-2.97(\mathrm{~m}, 2 \mathrm{H}), 2.88-2.75(\mathrm{~m}, 1 \mathrm{H}), 1.94(\mathrm{~s}, 1 \mathrm{H})$; Enantiomeric excess was 
determined by HPLC for the corresponding acetamide, Chiralpak AD-H column, $\mathrm{Hex} / \mathrm{IPA}=95: 5,1 \mathrm{~mL} / \mathrm{min}$.<smiles>COc1cccc(C2NCCc3ccccc32)c1</smiles>

(S)-1-(3-methoxyphenyl)-1,2,3,4-tetrahydroisoquinoline (10e): ${ }^{8}$ yellow oil; $117.1 \mathrm{mg}$, 98\% yield; ${ }^{1} \mathbf{H}$ NMR (400 MHz, $\left.\mathrm{CDCl}_{3}\right) \delta$ 7.28-7.20 (m, 1H), 7.18-7.10 (m, 2H), 7.08-7.00 (m, 1H), 6.90-6.76 (m, 4H), $5.08(\mathrm{~s}, 1 \mathrm{H}), 3.77(\mathrm{~s}, 3 \mathrm{H}), 3.33-3.22(\mathrm{~m}, 1 \mathrm{H})$, 3.14-2.97 (m, 2H), 2.87-2.77 (m, 1H), $2.04(\mathrm{~s}, 1 \mathrm{H})$; Enantiomeric excess was determined by HPLC for the corresponding acetamide, Chiralpak OD-H column, Hex/IPA $=90: 10,1 \mathrm{~mL} / \mathrm{min}$.<smiles>Cc1cccc(C2NCCc3ccccc32)c1</smiles>

(S)-1-(3-methyphenyl)-1,2,3,4-tetrahydroisoquinoline (10f): ${ }^{5}$ white solid; $109.3 \mathrm{mg}$, 98\% yield; ${ }^{1} \mathbf{H}$ NMR (400 MHz, $\left.\mathrm{CDCl}_{3}\right) \delta$ 7.24-7.18 (m, 1H), 7.17-7.12 (m, 2H), 7.11-7.07 (m, 2H), 7.07-6.99 (m, 2H), $6.76(\mathrm{~d}, J=7.7 \mathrm{~Hz}, 1 \mathrm{H}), 5.06(\mathrm{~s}, 1 \mathrm{H})$, 3.24-3.22 (m, 1H), 3.14-3.00 (m, 2H), 2.88-2.78 (m, 1H), $2.32(\mathrm{~s}, 3 \mathrm{H}), 2.06(\mathrm{~s}, 1 \mathrm{H})$; Enantiomeric excess was determined by HPLC for the corresponding acetamide, Chiralpak AD-H column, Hex/IPA = 90:10, $1 \mathrm{~mL} / \mathrm{min}$.<smiles>Fc1ccc(C2NCCc3ccccc32)cc1</smiles>

(S)-1-(4-fluorophenyl)-1,2,3,4-tetrahydroisoquinoline (10g): ${ }^{7}$ white solid; $112.4 \mathrm{mg}$, 99\% yield; ${ }^{1} \mathbf{H}$ NMR (400 MHz, $\left.\mathrm{CDCl}_{3}\right) \delta$ 7.27-7.19 (m, 2H), 7.18-7.12 (m, 2H), 7.07-6.95 (m, 3H), $6.72(\mathrm{~d}, J=7.7 \mathrm{~Hz}, 1 \mathrm{H}), 5.08(\mathrm{~s}, 1 \mathrm{H}), 3.31-3.19(\mathrm{~m}, 1 \mathrm{H})$, 3.14-2.96 (m, 2H), 2.87-2.77 (m, 1H), $1.94(\mathrm{~s}, 1 \mathrm{H})$; Enantiomeric excess was 
determined by HPLC for the corresponding acetamide, Chiralpak AD-H column, $\mathrm{Hex} / \mathrm{IPA}=95: 5,1 \mathrm{~mL} / \mathrm{min}$.<smiles>Cc1ccc(C2NCCc3ccccc32)cc1</smiles>

(S)-1-(4-methyphenyl)-1,2,3,4-tetrahydroisoquinoline (10h): ${ }^{5}$ white solid; $110.4 \mathrm{mg}$, 99\% yield; ${ }^{1} \mathbf{H}$ NMR (400 MHz, $\left.\mathrm{CDCl}_{3}\right) \delta$ 7.22-7.14 (m, 6H), 7.12-7.04 (m, 1H), 6.81 $(\mathrm{d}, J=7.7 \mathrm{~Hz}, 1 \mathrm{H}), 5.14(\mathrm{~s}, 1 \mathrm{H}), 3.37-.25(\mathrm{~m}, 1 \mathrm{H}), 3.18-3.01(\mathrm{~m}, 2 \mathrm{H}), 2.93-2.81(\mathrm{~m}$, 1H), 2.48-4-2.41 (m, 1H), $2.39(\mathrm{~s}, 3 \mathrm{H})$; Enantiomeric excess was determined by HPLC for the corresponding acetamide, Chiralpak AD-H column, Hex/IPA = 95:5, 1 $\mathrm{mL} / \mathrm{min}$.<smiles>FC(F)(F)c1ccc(C2NCCc3ccccc32)cc1</smiles>

(S)-1-(4-(trifluoromethyl)phenyl)-1,2,3,4-tetrahydroisoquinoline (10i): ${ }^{5}$ white solid; $137.1 \mathrm{mg}, 99 \%$ yield; ${ }^{1} \mathbf{H}$ NMR $\left(400 \mathrm{MHz}, \mathrm{CDCl}_{3}\right) \delta 7.58(\mathrm{~d}, J=8.0 \mathrm{~Hz}, 2 \mathrm{H}), 7.39$ (d, $J=8.0 \mathrm{~Hz}, 2 \mathrm{H}), 7.17(\mathrm{~d}, J=4.4 \mathrm{~Hz}, 2 \mathrm{H}), 7.10-7.00(\mathrm{~m}, 1 \mathrm{H}), 6.70(\mathrm{~d}, J=7.8 \mathrm{~Hz}$, 1H), $5.17(\mathrm{~s}, 1 \mathrm{H}), 3.29-3.18(\mathrm{~m}, 1 \mathrm{H}), 3.14-2.97$ (m, 2H), 2.89-2.77 (m, 1H), 2.05 (s, 1H); Enantiomeric excess was determined by HPLC for the corresponding acetamide, Chiralpak AD-H column, Hex/IPA = 95:5, $1 \mathrm{~mL} / \mathrm{min}$.<smiles>COc1cc2c(cc1OC)C(c1ccccc1)NCC2</smiles>

(S)-6,7-dimethoxy-1-phenyl-1,2,3,4-tetrahydroisoquinoline (10j): ${ }^{5}$ white solid; 131.8 mg, 98\% yield; ${ }^{1} \mathbf{H}$ NMR (400 MHz, $\mathrm{CDCl}_{3}$ ) $\delta$ 7.39-7.30 (m, 3H), 7.28-7.25 (m, 1H), $6.66(\mathrm{~s}, 1 \mathrm{H}), 6.27(\mathrm{~s}, 1 \mathrm{H}), 5.08(\mathrm{~s}, 1 \mathrm{H}), 3.90(\mathrm{~s}, 3 \mathrm{H}), 3.66(\mathrm{~s}, 3 \mathrm{H}), 3.30-3.20(\mathrm{~m}, 1 \mathrm{H})$, 3.12-3.03 (m, 1H), 3.01-2.89 (m, 1H), 2.83-2.72 (m, 1H), $2.14(\mathrm{~s}, 1 \mathrm{H})$; Enantiomeric 
excess was determined by HPLC for the corresponding acetamide, Chiralpak AD-H column, Hex/IPA = 90:10, $1 \mathrm{~mL} / \mathrm{min}$.<smiles>COc1cc2c(cc1OC)[C@@H](c1ccccc1F)NCC2</smiles>

(R)-1-(2-fluorophenyl)-6,7-dimethoxy-1,2,3,4-tetrahydroisoquinoline $(\mathbf{1 0 k}):^{9}$ brown oil; $139.2 \mathrm{mg}, 97 \%$ yield; ${ }^{1} \mathbf{H}$ NMR (400 MHz, $\left.\mathrm{CDCl}_{3}\right) \delta$ 7.30-7.20 (m, 1H), 7.14-6.94 (m, 3H), $6.64(\mathrm{~s}, 1 \mathrm{H}), 6.28(\mathrm{~s}, 1 \mathrm{H}), 5.45(\mathrm{~s}, 1 \mathrm{H}), 3.88(\mathrm{~s}, 3 \mathrm{H}), 3.67(\mathrm{~s}, 3 \mathrm{H})$, 3.19-3.10 (m, 1H), 3.08-3.00 (m, 1H), 2.93-2.74 (m, 2H), 1.97 (s, 1H); Enantiomeric excess was determined by HPLC for the corresponding acetamide, Chiralpak AD-H column, Hex/IPA = 92:8, $1 \mathrm{~mL} / \mathrm{min}$.<smiles>COc1cc2c(cc1OC)[C@@H](c1ccccc1OC)NCC2</smiles>

(R)-6,7-dimethoxy-1-(2-methoxyphenyl)-1,2,3,4-tetrahydroisoquinoline (101): ${ }^{9}$ brown oil; 140.5 mg, 94\% yield; ${ }^{1}$ H NMR (400 MHz, $\left.\mathrm{CDCl}_{3}\right) \delta$ 7.25-7.20 (m, 1H), 6.93 (d, $J$ $=7.8 \mathrm{~Hz}, 1 \mathrm{H}), 6.85-6.76(\mathrm{~m}, 2 \mathrm{H}), 6.64(\mathrm{~s}, 1 \mathrm{H}), 6.32(\mathrm{~s}, 1 \mathrm{H}), 5.49(\mathrm{~s}, 1 \mathrm{H}), 3.89(\mathrm{~s}$, $3 \mathrm{H}), 3.88(\mathrm{~s}, 3 \mathrm{H}), 3.68(\mathrm{~s}, 3 \mathrm{H}), 3.09-2.93(\mathrm{~m}, 2 \mathrm{H}), 2.89-2.72(\mathrm{~m}, 2 \mathrm{H}), 2.09(\mathrm{~s}, 1 \mathrm{H})$; Enantiomeric excess was determined by HPLC for the corresponding acetamide, Chiralpak AD-H column, Hex/IPA = 90:10, $1 \mathrm{~mL} / \mathrm{min}$.<smiles>COc1cc2c(cc1OC)[C@@H](c1ccccc1C)NCC2</smiles>

(S)-6,7-dimethoxy-1-(2-methyphenyl)-1,2,3,4-tetrahydroisoquinoline (10m): ${ }^{9}$ brown oil; $133.0 \mathrm{mg}, 94 \%$ yield; ${ }^{1} \mathbf{H}$ NMR (400 MHz, $\left.\mathrm{CDCl}_{3}\right) \delta 7.22-7.14(\mathrm{~m}, 2 \mathrm{H})$, 7.12-7.07 (m, 1H), $6.98(\mathrm{~d}, J=7.4 \mathrm{~Hz}, 1 \mathrm{H}), 6.63(\mathrm{~s}, 1 \mathrm{H}), 6.19(\mathrm{~s}, 1 \mathrm{H}), 5.28(\mathrm{~s}, 1 \mathrm{H})$, $3.88(\mathrm{~s}, 3 \mathrm{H}), 3.63(\mathrm{~s}, 3 \mathrm{H}), 3.26-3.18(\mathrm{~m}, 1 \mathrm{H}), 3.09-2.99(\mathrm{~m}, 1 \mathrm{H}), 2.97-2.85(\mathrm{~m}, 1 \mathrm{H})$, 
2.80-2.70 (m, 1H), $2.43(\mathrm{~s}, 3 \mathrm{H}), 1.94(\mathrm{~s}, 1 \mathrm{H})$; Enantiomeric excess was determined by HPLC for the corresponding acetamide, Chiralpak AD-H column, Hex/IPA = 92:8, 1 $\mathrm{mL} / \mathrm{min}$.<smiles>COc1cc2c(cc1OC)C(c1cccc(F)c1)NCC2</smiles>

(S)-1-(3-fluorophenyl)-6,7-dimethoxy-1,2,3,4-tetrahydroisoquinoline (10n): ${ }^{12}$ white solid; 140.6 mg, 98\% yield; ${ }^{1} \mathbf{H}$ NMR (400 MHz, $\left.\mathrm{CDCl}_{3}\right) \delta$ 7.34-7.28 (m, 1H), 7.09 (d, $J=7.6 \mathrm{~Hz}, 1 \mathrm{H}), 7.03-6.94(\mathrm{~m}, 2 \mathrm{H}), 6.66(\mathrm{~s}, 1 \mathrm{H}), 6.26(\mathrm{~s}, 1 \mathrm{H}), 5.08(\mathrm{~s}, 1 \mathrm{H}), 3.90(\mathrm{~s}$, $3 \mathrm{H}), 3.68(\mathrm{~s}, 3 \mathrm{H}), 3.26-3.17$ (m, 1H), 3.11-3.01 (m, 1H), 2.99-2.88 (m, 1H), 2.84-2.74 $(\mathrm{m}, 1 \mathrm{H}), 2.18(\mathrm{~s}, 1 \mathrm{H})$; Enantiomeric excess was determined by HPLC for the corresponding acetamide, Chiralpak AD-H column, Hex/IPA = 90:10, $1 \mathrm{~mL} / \mathrm{min}$.<smiles>COc1cccc(C2NCCc3cc(OC)c(OC)cc32)c1</smiles>

(S)-6,7-dimethoxy-1-(3-methoxyphenyl)-1,2,3,4-tetrahydroisoquinoline $(\mathbf{1 0 0}): 8$ yellow solid; $145.0 \mathrm{mg}, 97 \%$ yield; ${ }^{1} \mathbf{H}$ NMR (400 MHz, $\left.\mathrm{CDCl}_{3}\right) \delta 7.29-7.18(\mathrm{~m}, 1 \mathrm{H})$, 6.88-6.74 (m, 3H), $6.63(\mathrm{~s}, 1 \mathrm{H}), 6.27(\mathrm{~s}, 1 \mathrm{H}), 5.02(\mathrm{~s}, 1 \mathrm{H}), 3.87(\mathrm{~s}, 3 \mathrm{H}), 3.78(\mathrm{~s}, 3 \mathrm{H})$, $3.65(\mathrm{~s}, 3 \mathrm{H}), 3.26-3.16(\mathrm{~m}, 1 \mathrm{H}), 3.08-2.98(\mathrm{~m}, 1 \mathrm{H}), 2.97-2.86(\mathrm{~m}, 1 \mathrm{H}), 2.80-2.69(\mathrm{~m}$, 1H), $1.93(\mathrm{~s}, 1 \mathrm{H})$; Enantiomeric excess was determined by HPLC for the corresponding acetamide, Chiralpak OD-H column, Hex/IPA = 90:10, $1 \mathrm{~mL} / \mathrm{min}$.<smiles>COc1cc2c(cc1OC)[C@H](c1cccc(C)c1)NCC2</smiles>

(S)-6,7-dimethoxy-1-(3-methyphenyl)-1,2,3,4-tetrahydroisoquinoline (10p): ${ }^{9}$ yellow solid; $138.7 \mathrm{mg}, 98 \%$ yield; ${ }^{1} \mathbf{H}$ NMR (400 $\left.\mathrm{MHz} \mathrm{CDCl}_{3}\right) \delta$ 7.26-7.20 (m, 1H), 7.14-7.08 (m, 2H), 7.07-7.02 (m, 1H), $6.66(\mathrm{~s}, 1 \mathrm{H}), 6.28(\mathrm{~s}, 1 \mathrm{H}), 5.05(\mathrm{~s}, 1 \mathrm{H}), 3.90(\mathrm{~s}$, 
$3 \mathrm{H}), 3.67(\mathrm{~s}, 3 \mathrm{H}), 3.28-3.19(\mathrm{~m}, 1 \mathrm{H}), 3.11-2.90(\mathrm{~m}, 2 \mathrm{H}), 2.85-2.73(\mathrm{~m}, 1 \mathrm{H}), 2.35(\mathrm{~s}$, $3 \mathrm{H}), 2.19(\mathrm{~s}, 1 \mathrm{H})$; Enantiomeric excess was determined by HPLC for the corresponding acetamide, Chiralpak AD-H column, Hex/IPA = 90:10, $1 \mathrm{~mL} / \mathrm{min}$.<smiles>COc1cc2c(cc1OC)C(c1ccc(F)cc1)NCC2</smiles>

(S)-1-(4-fluorophenyl)-6,7-dimethoxy-1,2,3,4-tetrahydroisoquinoline (10q):9 yellow solid; $142.1 \mathrm{mg}, 99 \%$ yield; ${ }^{1} \mathbf{H}$ NMR (400 MHz, $\left.\mathrm{CDCl}_{3}\right) \delta$ 7.27-7.22 (m, 2H), $7.03(\mathrm{t}$, $J=8.7 \mathrm{~Hz}, 2 \mathrm{H}), 6.65(\mathrm{~s}, 1 \mathrm{H}), 6.22(\mathrm{~s}, 1 \mathrm{H}), 5.07(\mathrm{~s}, 1 \mathrm{H}), 3.90(\mathrm{~s}, 3 \mathrm{H}), 3.67(\mathrm{~s}, 3 \mathrm{H})$, 3.28-3.16 (m, 1H), 3.12-3.02 (m, 1H), 3.01-2.89 (m, 1H), 2.82-2.72 (m, 1H), $2.15(\mathrm{~s}$, $1 \mathrm{H})$; Enantiomeric excess was determined by HPLC for the corresponding acetamide, Chiralpak AD-H column, Hex/IPA = 85:15, $1 \mathrm{~mL} / \mathrm{min}$.<smiles>COc1cc2c(cc1OC)C(c1ccc(Cl)cc1)NCC2</smiles>

(S)-1-(4-chlorophenyl)-6,7-dimethoxy-1,2,3,4-tetrahydroisoquinoline (10r): ${ }^{7}$ colorless oil; 149.9 mg, 99\% yield; ${ }^{1}$ H NMR (400 MHz, $\left.\mathrm{CDCl}_{3}\right) \delta 7.28(\mathrm{~d}, J=8.4 \mathrm{~Hz}, 2 \mathrm{H})$, $7.19(\mathrm{~d}, J=8.5 \mathrm{~Hz}, 2 \mathrm{H}), 6.63(\mathrm{~s}, 1 \mathrm{H}), 6.20(\mathrm{~s}, 1 \mathrm{H}), 5.02(\mathrm{~s}, 1 \mathrm{H}), 3.86(\mathrm{~s}, 3 \mathrm{H}), 3.64(\mathrm{~s}$, $3 \mathrm{H}), 3.23-3.13(\mathrm{~m}, 1 \mathrm{H}), 3.08-2.98(\mathrm{~m}, 1 \mathrm{H}), 2.96-2.85(\mathrm{~m}, 1 \mathrm{H}), 2.79-2.66(\mathrm{~m}, 1 \mathrm{H})$, $2.05(\mathrm{~s}, 1 \mathrm{H})$; Enantiomeric excess was determined by HPLC for the corresponding acetamide, Chiralpak AD-H column, Hex/IPA = 80:20, $1 \mathrm{~mL} / \mathrm{min}$.<smiles>COc1ccc(C2NCCc3cc(OC)c(OC)cc32)cc1</smiles>

(S)-6,7-dimethoxy-1-(4-methoxyphenyl)-1,2,3,4-tetrahydroisoquinoline (10s): ${ }^{9}$ yellow 
solid; $146.5 \mathrm{mg}$, 98\% yield; ${ }^{1} \mathbf{H}$ NMR (400 MHz, $\left.\mathrm{CDCl}_{3}\right) \delta$ 7.22-7.14 (m, 2H), 6.90-6.80 (m, 2H), $6.62(\mathrm{~s}, 1 \mathrm{H}), 6.25(\mathrm{~s}, 1 \mathrm{H}), 5.00(\mathrm{~s}, 1 \mathrm{H}), 3.87(\mathrm{~s}, 3 \mathrm{H}), 3.80(\mathrm{~s}, 3 \mathrm{H})$, $3.65(\mathrm{~s}, 3 \mathrm{H}), 3.26-3.16(\mathrm{~m}, 1 \mathrm{H}), 3.10-2.98(\mathrm{~m}, 1 \mathrm{H}), 2.97-2.86(\mathrm{~m}, 1 \mathrm{H}), 2.79-2.69(\mathrm{~m}$, 1H), $1.91(\mathrm{~s}, 1 \mathrm{H})$; Enantiomeric excess was determined by HPLC for the corresponding acetamide, Chiralpak AD-H column, Hex/IPA $=85: 15,1 \mathrm{~mL} / \mathrm{min}$.<smiles>COc1cc2c(cc1OC)C(c1ccc(C)cc1)NCC2</smiles>

(S)-6,7-dimethoxy-1-(4-methoxyphenyl)-1,2,3,4-tetrahydroisoquinoline (10t): ${ }^{8}$ yellow solid; $138.7 \mathrm{mg}, 98 \%$ yield; ${ }^{1} \mathbf{H}$ NMR (400 MHz, $\left.\mathrm{CDCl}_{3}\right) \delta$ 7.22-7.10 (m, 4H), 6.65 (s, 1H), $6.28(\mathrm{~s}, 1 \mathrm{H}), 5.05(\mathrm{~s}, 1 \mathrm{H}), 3.90(\mathrm{~s}, 3 \mathrm{H}), 3.67(\mathrm{~s}, 3 \mathrm{H}), 3.26-3.17(\mathrm{~m}, 1 \mathrm{H})$, 3.10-3.01 (m, 1H), 2.99-2.89 (m, 1H), 2.82-2.72 (m, 1H), $2.36(\mathrm{~s}, 3 \mathrm{H}), 2.13(\mathrm{~s}, 1 \mathrm{H})$; Enantiomeric excess was determined by HPLC for the corresponding acetamide, Chiralpak AD-H column, Hex/IPA = 90:10, $1 \mathrm{~mL} / \mathrm{min}$.<smiles>COc1ccc(C2NCCc3cc(OC)c(OC)cc32)cc1OC</smiles>

(S)-1-(3,4-dimethoxyphenyl)-6,7-dimethoxy-1,2,3,4-tetrahydroisoquinoline (10u): ${ }^{5}$ white solid; 162.9 mg, 99\% yield; ${ }^{1} \mathbf{H}$ NMR (400 MHz, $\left.\mathrm{CDCl}_{3}\right) \delta$ 6.87-6.74 (m, 3H), $6.63(\mathrm{~s}, 1 \mathrm{H}), 6.27(\mathrm{~s}, 1 \mathrm{H}), 4.98(\mathrm{~s}, 1 \mathrm{H}), 3.86(\mathrm{~s}, 6 \mathrm{H}), 3.82(\mathrm{~s}, 3 \mathrm{H}), 3.64(\mathrm{~s}, 3 \mathrm{H})$, 3.30-3.15 (m, 1H), 3.10-3.00 (m, 1H), 2.99-2.88 (m, 1H), 2.78-2.65 (m, 1H), $2.18(\mathrm{~s}$, $1 \mathrm{H})$; Enantiomeric excess was determined by HPLC for the corresponding acetamide, Chiralpak AD-H column, Hex/IPA = 80:20, $1 \mathrm{~mL} / \mathrm{min}$. 
<smiles>COc1cc2c(cc1OC)C(c1cc(OC)c(OC)c(OC)c1)NCC2</smiles>

(S)-6,7-dimethoxy-1-(3,4,5-trimethoxyphenyl)-1,2,3,4-tetrahydroisoquinoline (10v): ${ }^{5}$ white solid; $177.7 \mathrm{mg}$, 99\% yield; ${ }^{1} \mathbf{H}$ NMR (400 MHz, $\left.\mathrm{CDCl}_{3}\right) \delta 6.63(\mathrm{~s}, 1 \mathrm{H}), 6.50$ $(\mathrm{s}, 2 \mathrm{H}), 6.31(\mathrm{~s}, 1 \mathrm{H}), 4.98(\mathrm{~s}, 1 \mathrm{H}), 3.88(\mathrm{~s}, 3 \mathrm{H}), 3.85(\mathrm{~s}, 3 \mathrm{H}), 3.81(\mathrm{~s}, 6 \mathrm{H}), 3.68(\mathrm{~s}$, $3 \mathrm{H}), 3.30-3.16(\mathrm{~m}, 1 \mathrm{H}), 3.10-3.00(\mathrm{~m}, 1 \mathrm{H}), 3.00-2.85(\mathrm{~m}, 1 \mathrm{H}), 2.80-2.65(\mathrm{~m}, 1 \mathrm{H})$, $2.12(\mathrm{~s}, 1 \mathrm{H})$; Enantiomeric excess was determined by HPLC for the corresponding acetamide, Chiralpak AD-H column, Hex/IPA = 80:20, $1 \mathrm{~mL} / \mathrm{min}$.<smiles>COc1cc2c(cc1OC)C(C)NCC2</smiles>

(S)-6,7-dimethoxy-1-methyl-1,2,3,4-tetrahydroisoquinoline (10w): ${ }^{11}$ yellow oil; 101.4 mg, 98\% yield; ${ }^{1} \mathbf{H}$ NMR (400 MHz, $\left.\mathrm{CDCl}_{3}\right) \delta 6.63(\mathrm{~s}, 1 \mathrm{H}), 6.57$ (s, 1H), 4.10-4.00 $(\mathrm{m}, 1 \mathrm{H}), 3.86(\mathrm{~s}, 6 \mathrm{H}), 3.41-3.15(\mathrm{~m}, 1 \mathrm{H}), 3.06-2.94(\mathrm{~m}, 1 \mathrm{H}), 2.85-2.72(\mathrm{~m}, 1 \mathrm{H})$, 2.70-2.60 (m, 1H), $1.74(\mathrm{~s}, 1 \mathrm{H}), 1.44(\mathrm{~d}, J=6.7 \mathrm{~Hz}, 3 \mathrm{H})$; Enantiomeric excess was determined by HPLC for the corresponding acetamide, Chiralpak AD-H column, Hex/IPA $=90: 10,1 \mathrm{~mL} / \mathrm{min}$.<smiles>CCC1NCCc2cc(OC)c(OC)cc21</smiles>

(S)-6,7-dimethoxy-1-ethyl-1,2,3,4-tetrahydroisoquinoline (10x): ${ }^{11}$ yellow oil; 108.3 mg, 98\% yield; ${ }^{1} \mathbf{H}$ NMR (400 MHz, $\left.\mathrm{CDCl}_{3}\right) \delta 6.62(\mathrm{~s}, 1 \mathrm{H}), 6.57(\mathrm{~s}, 1 \mathrm{H}), 3.85(\mathrm{~s}, 6 \mathrm{H})$, 3.87-3.81 (m, 1H), 3.31-3.14 (m, 1H), 3.01-2.91 (m, 1H), 2.80-2.70 (m, 1H), 2.70-2.59 (m, 1H), 1.97-1.82 (m, 1H), 1.77-1.57 (m, 2H), $1.02(\mathrm{t}, J=7.4 \mathrm{~Hz}, 3 \mathrm{H})$; Enantiomeric excess was determined by HPLC for the corresponding acetamide, Chiralpak AD-H column, Hex/IPA = 92:8, $1 \mathrm{~mL} / \mathrm{min}$. 
Gram-scale catalytic hydrogenation: According to the above procedure, the catalyst solution was prepared with $[\operatorname{Ir}(\mathrm{COD}) \mathrm{Cl}]_{2}(1.6 \mathrm{mg}, 2.4 \mu \mathrm{mol})$, ligand $\left(R_{C}, S_{F c}, S_{a x}\right)-\mathbf{1 a}$ (3.6 mg, $5.4 \mu \mathrm{mol})$ and THF (1 mL) under nitrogen atmosphere. To a hydrogenation vessel was added a solution of imine substrates 9a ( $3 \mathrm{~g}, 14.5 \mathrm{mmol})$ in THF (60 mL) and $40 \% \mathrm{HBr}(0.22 \mathrm{~mL}, 1.45 \mathrm{mmol}, 10 \mathrm{~mol} \%)$. The resulting vessel was transferred to an autoclave, which was replaced with $50 \mathrm{~atm}$ of $\mathrm{H}_{2}$ for 3 times, and the reaction mixtures were stirred at $50{ }^{\circ} \mathrm{C}$ for $24 \mathrm{~h}$. After the reaction cooled to room temperature, the hydrogen gas was released slowly and the solution was concentrated. The residue was dissolved in EtOAc $(100 \mathrm{~mL})$, washed with saturated $\mathrm{NaHCO}_{3}$ solution, brine and dried over $\mathrm{Na}_{2} \mathrm{SO}_{4}$. The solvent was evaporated to afford the crude product, which was purified with chromatography (EtOAc/PE) to yield chiral 1-phenyl THIQ $(2.85 \mathrm{~g}, 94 \%, 98 \%$ ee $)$.

\section{NMR spectra and HRMS}



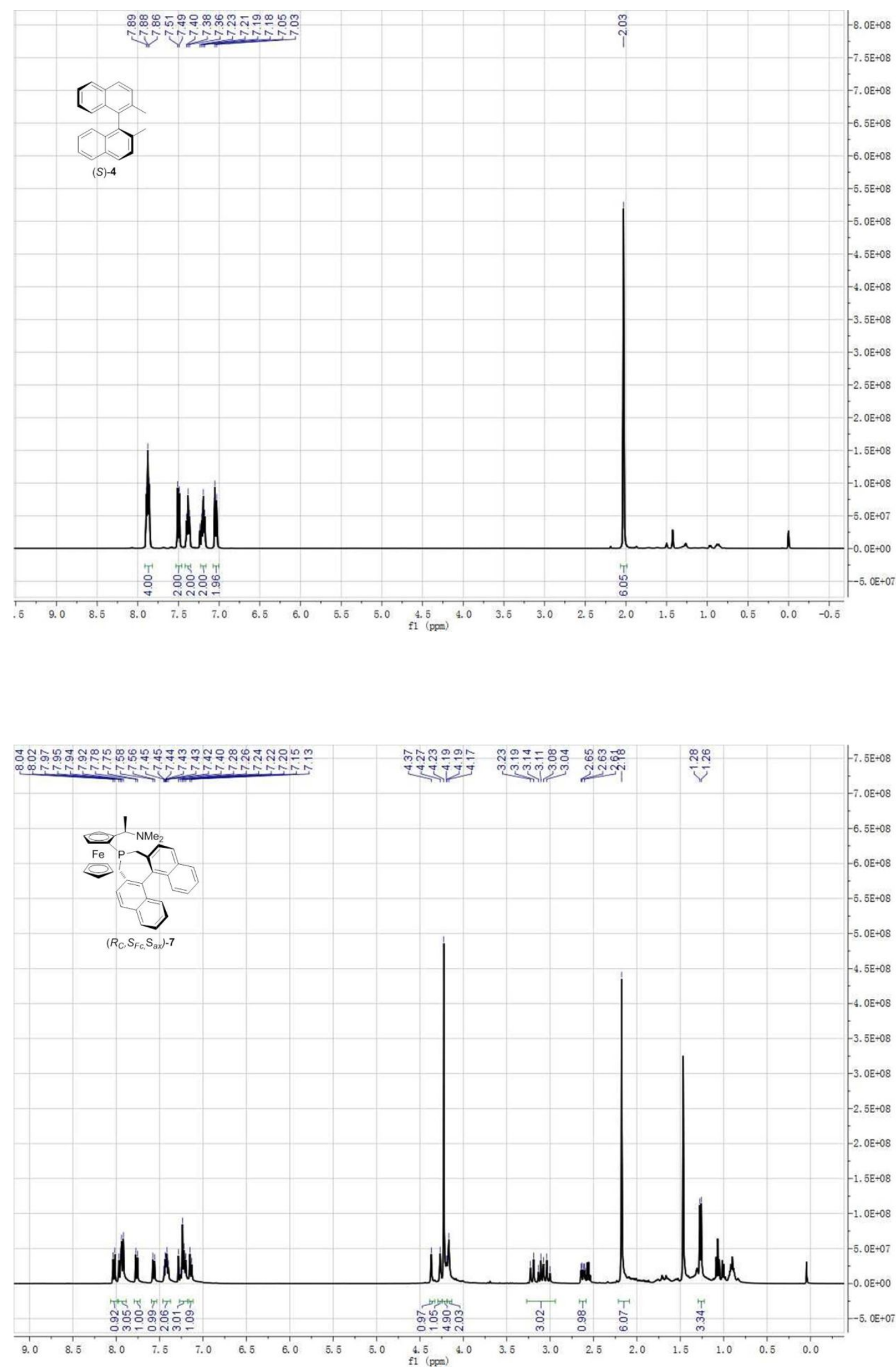

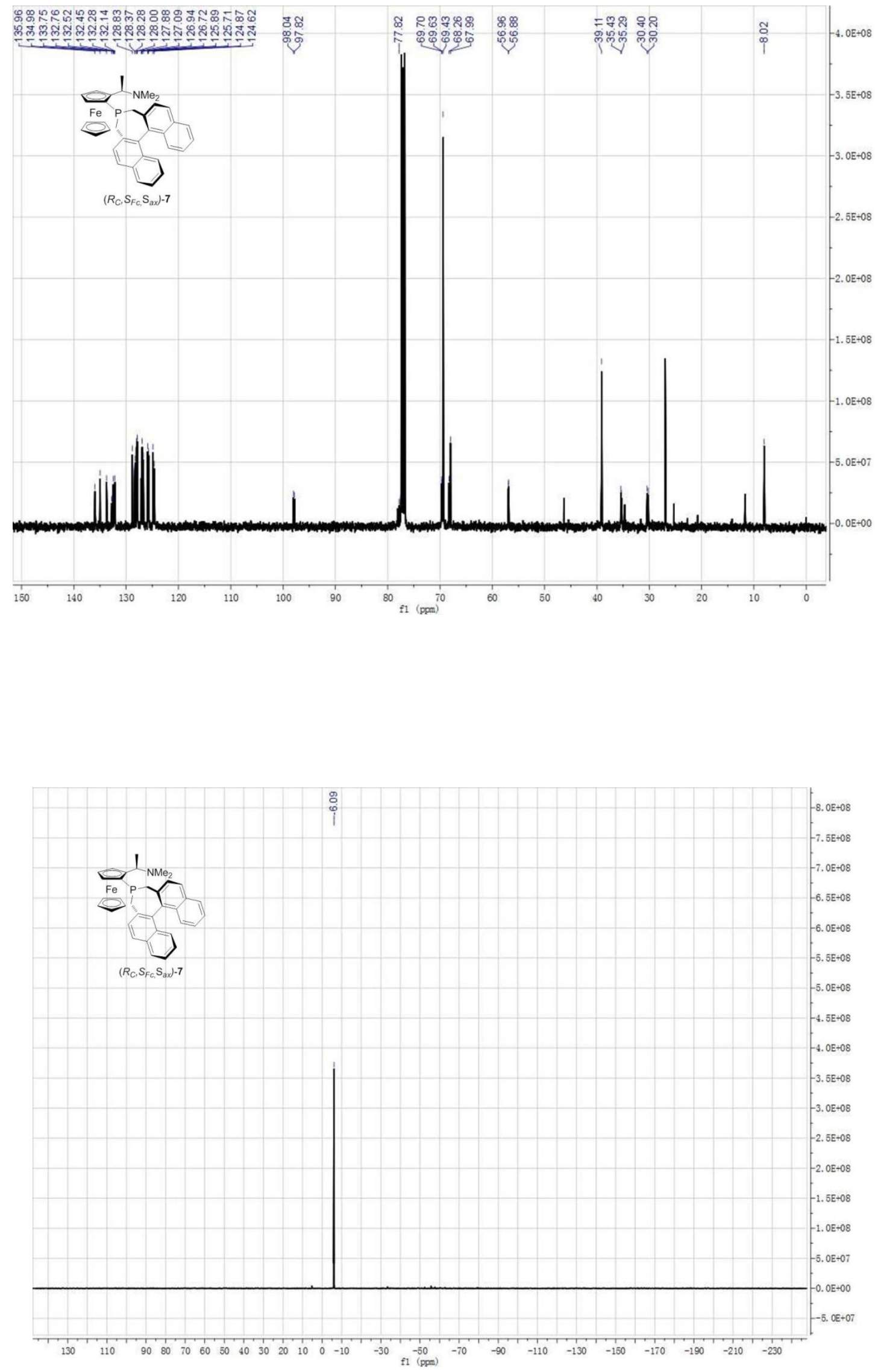

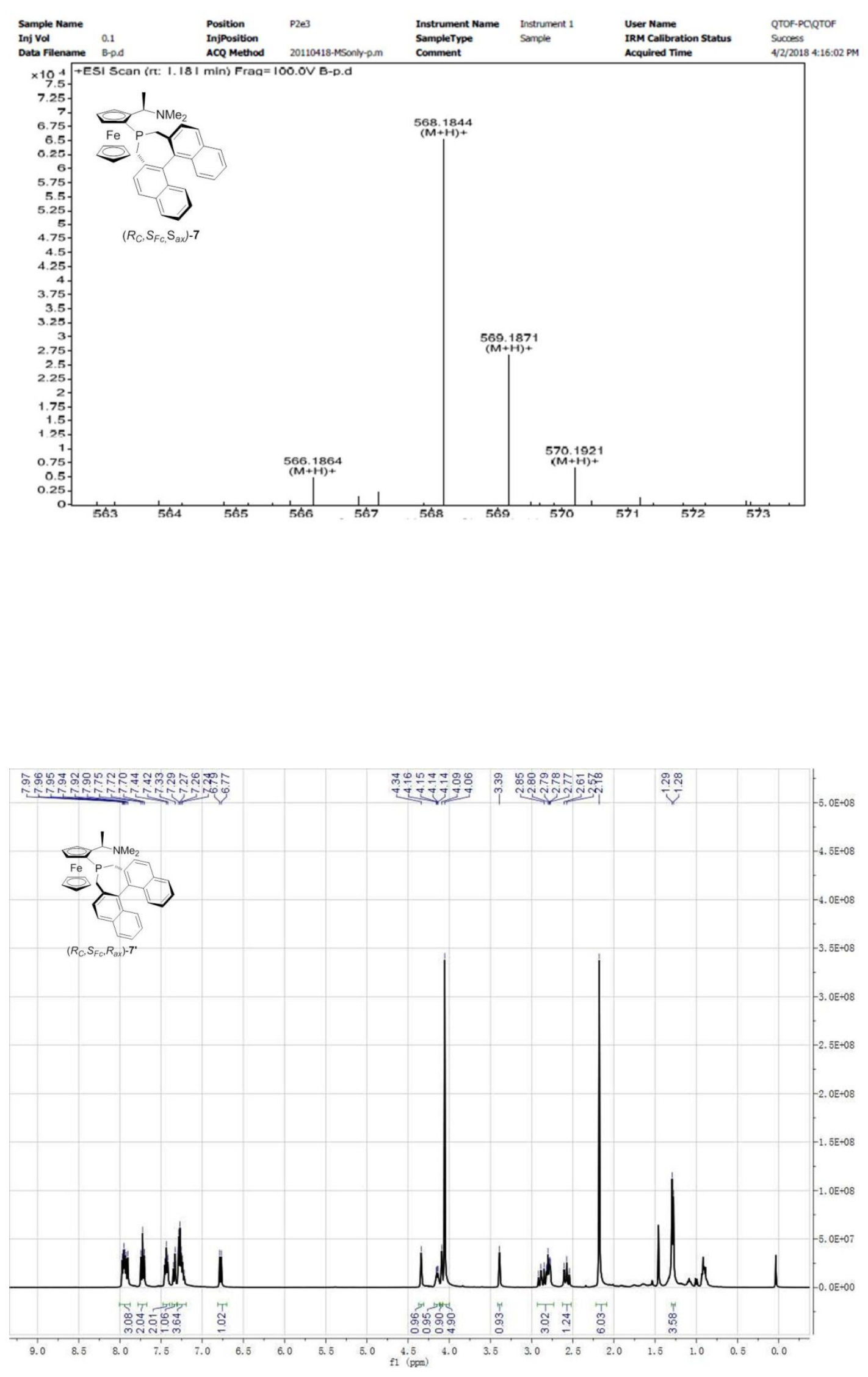

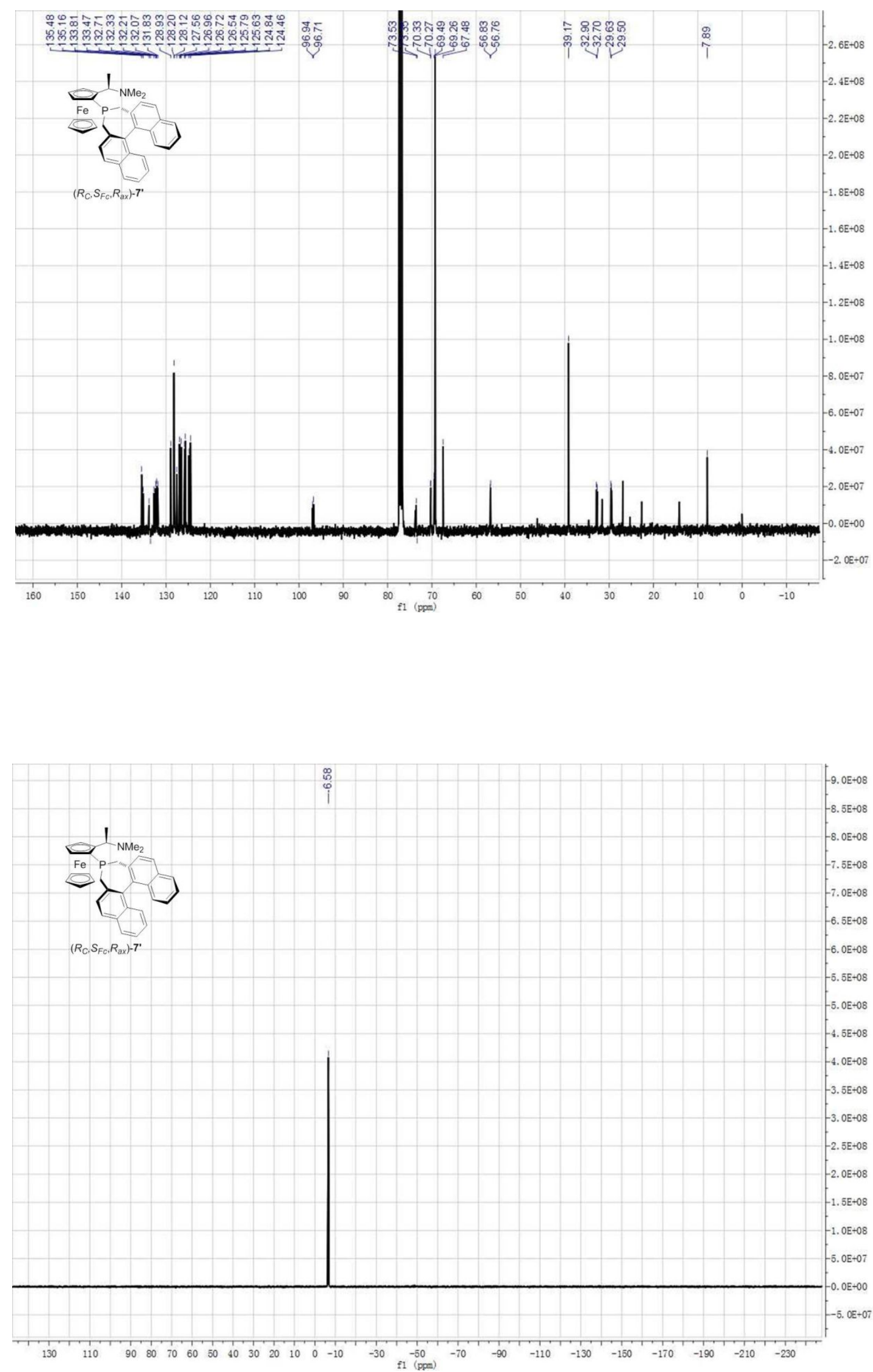

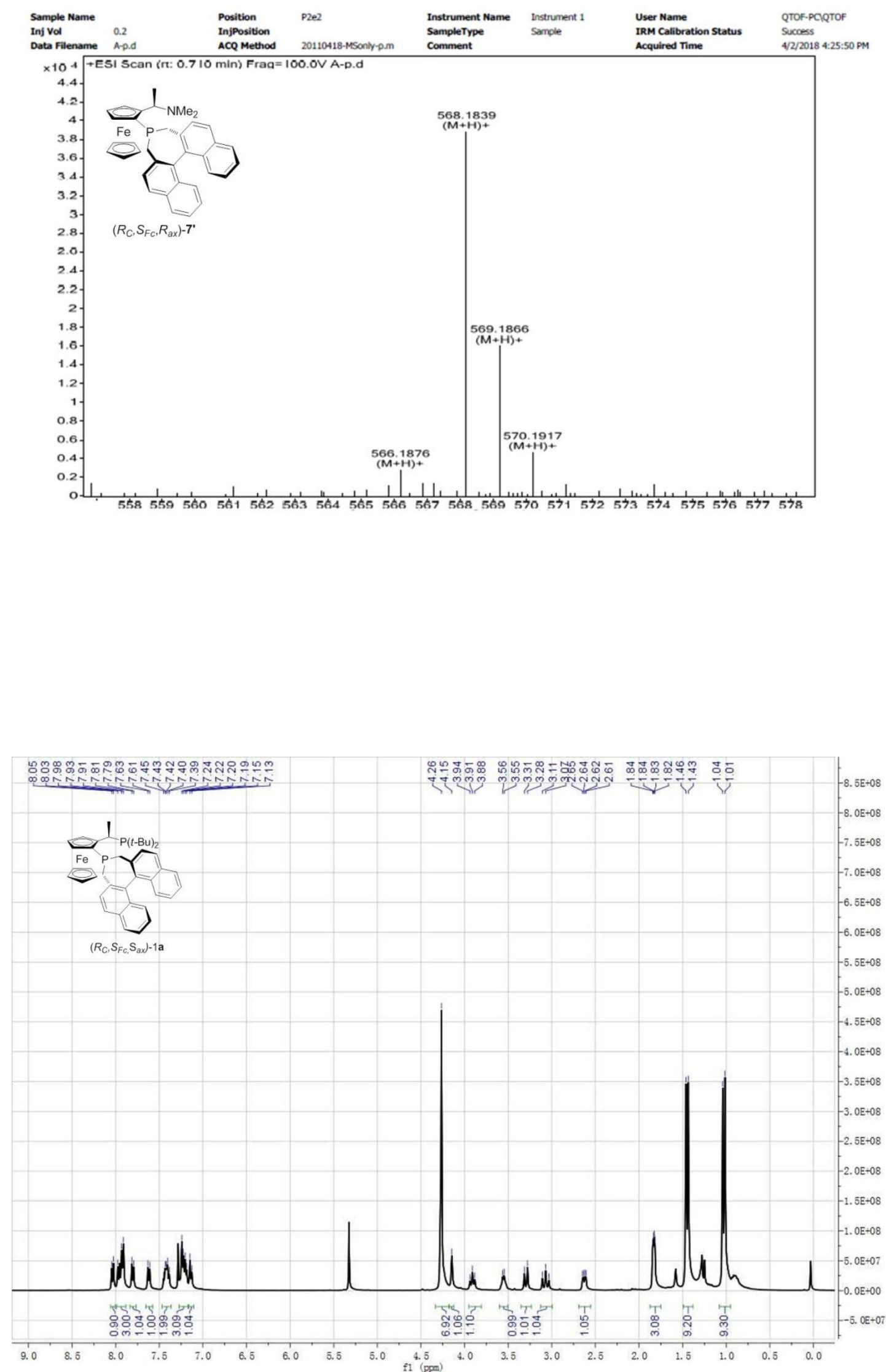

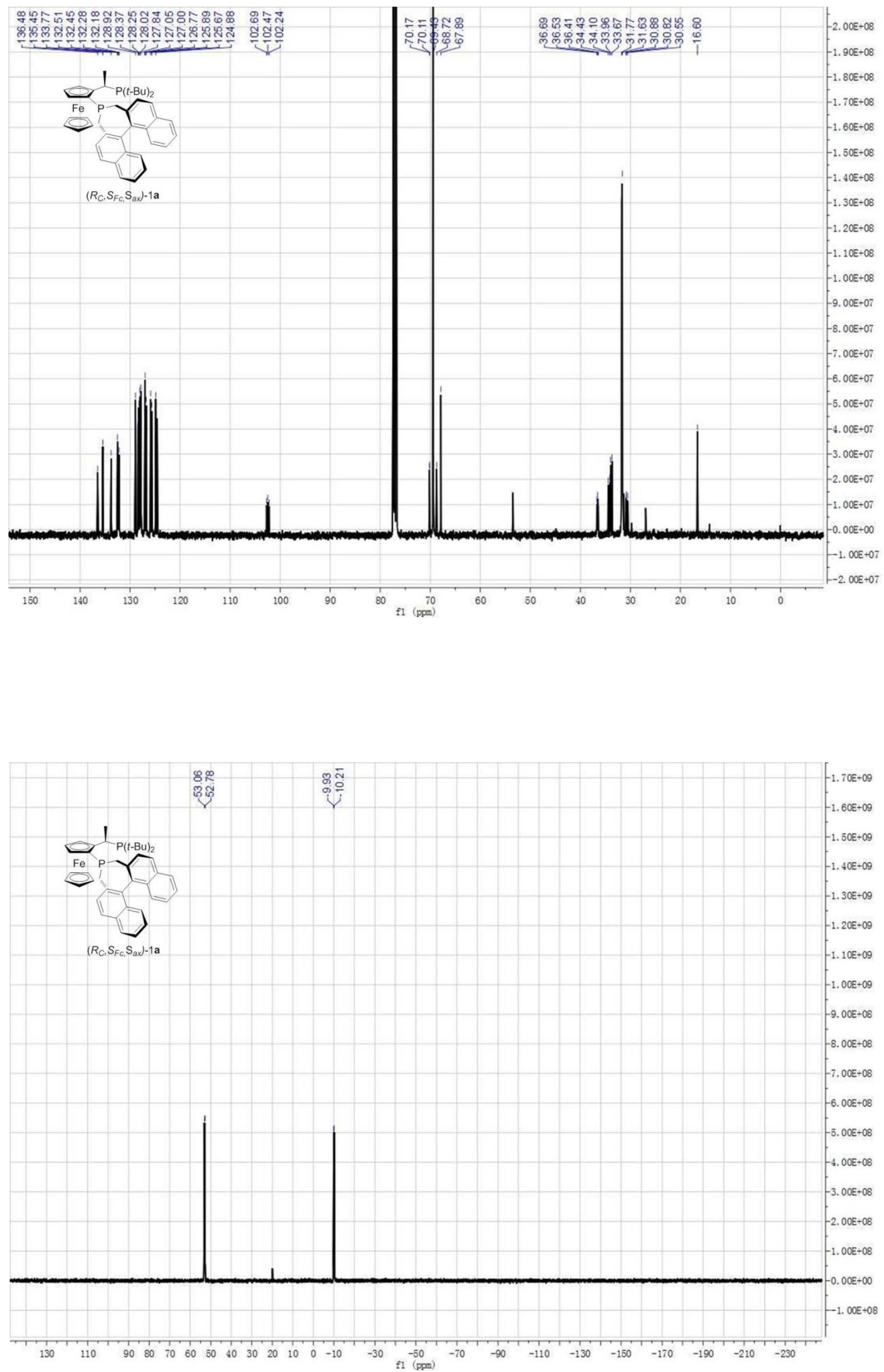

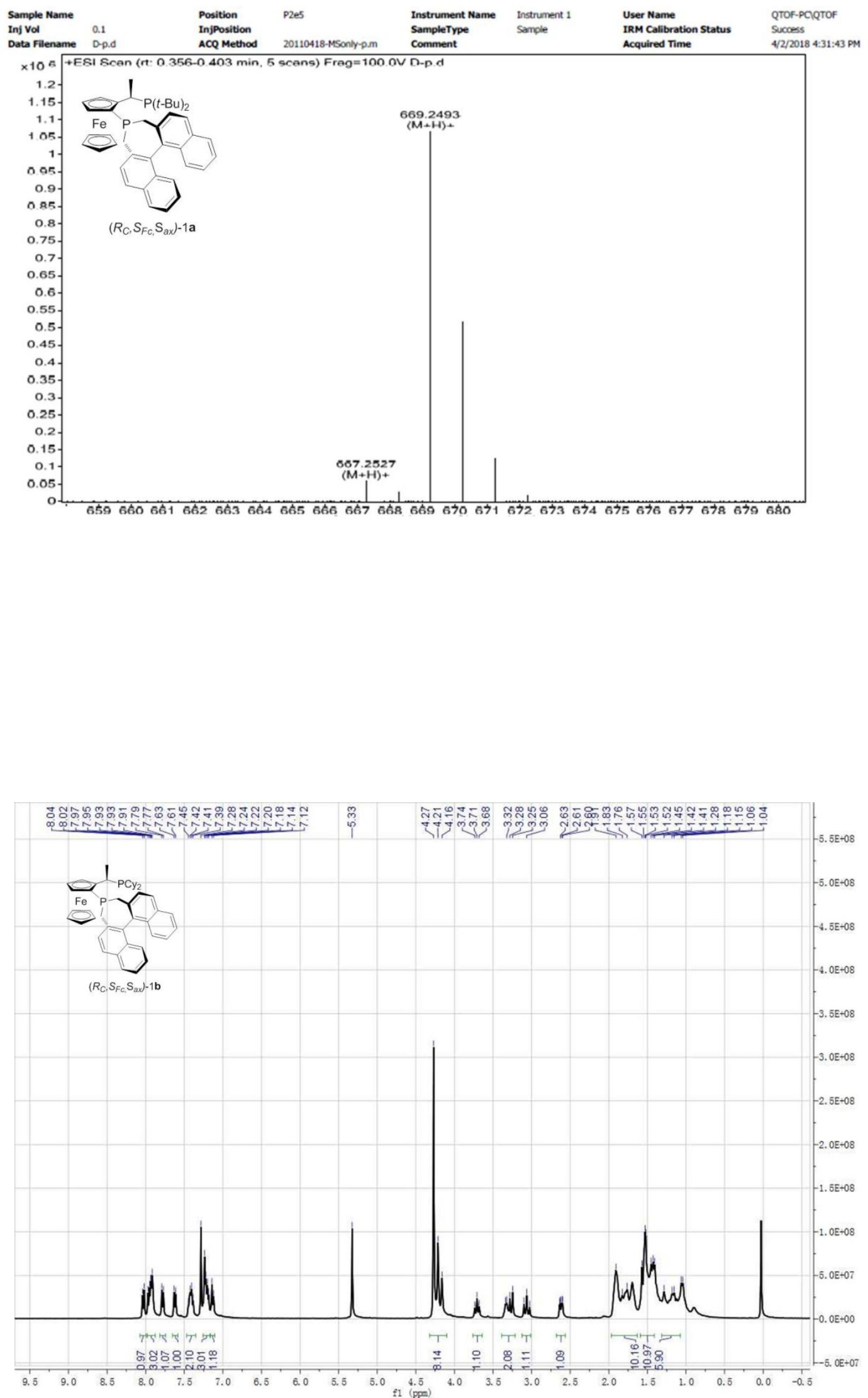

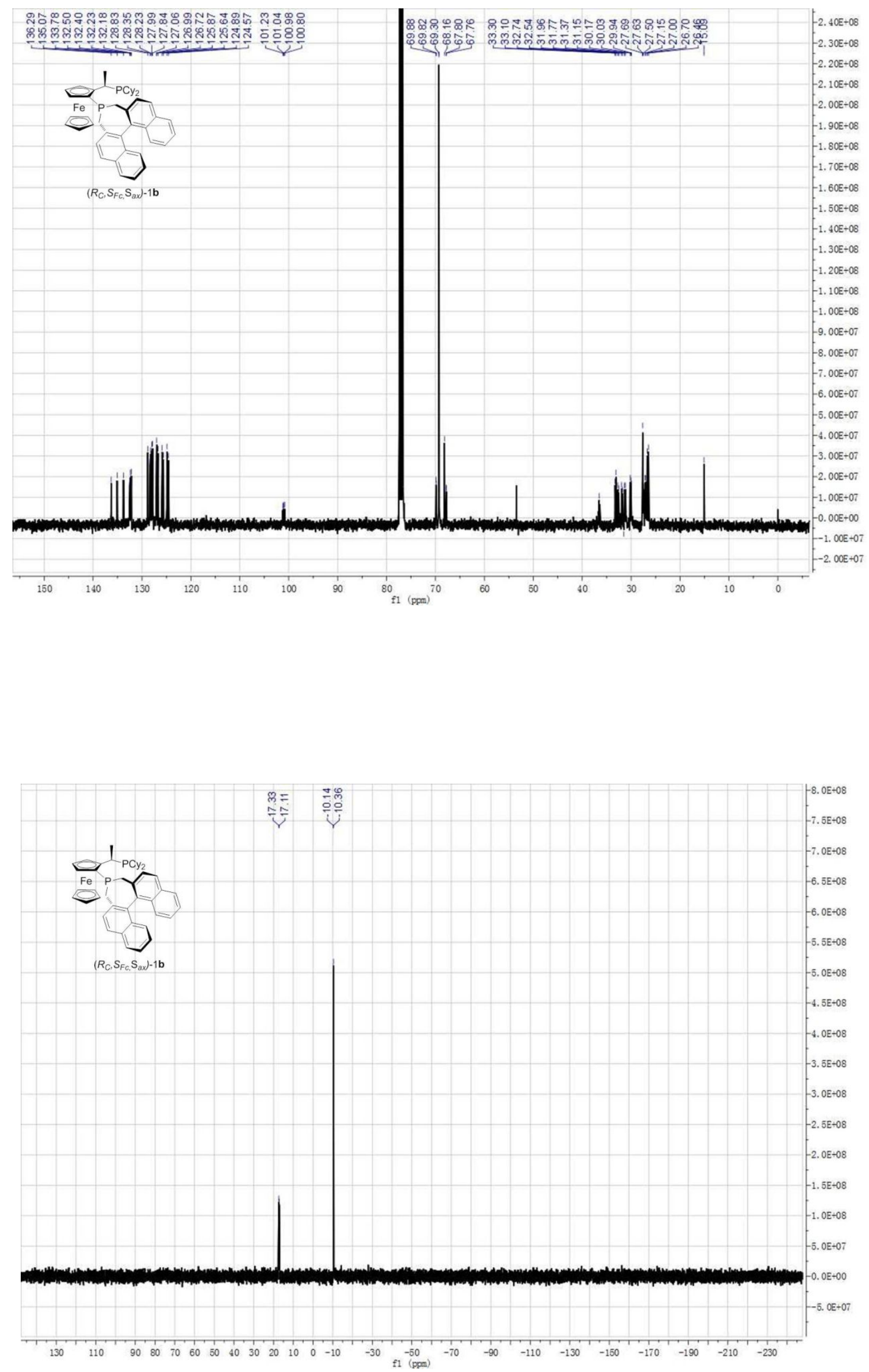

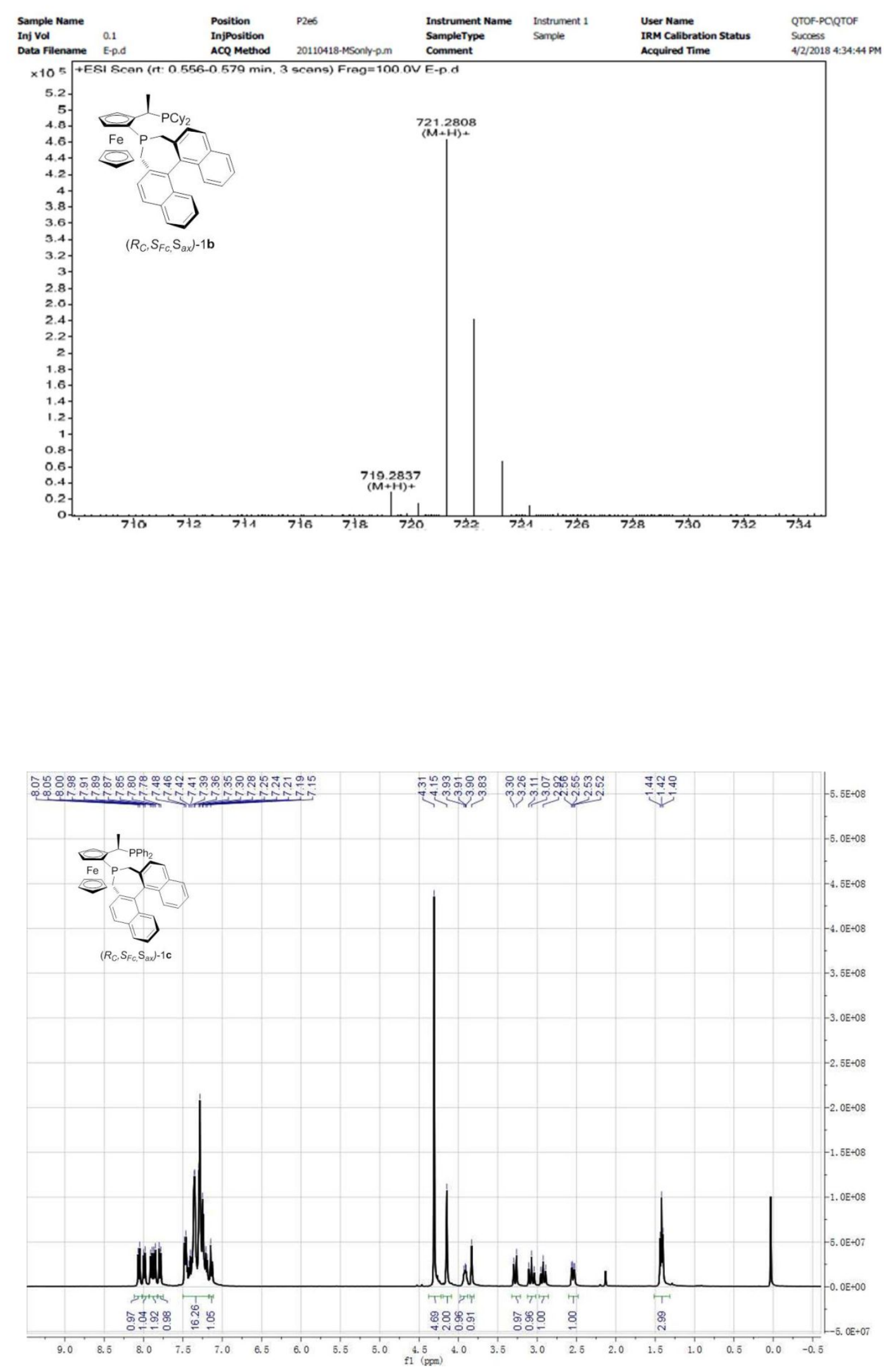

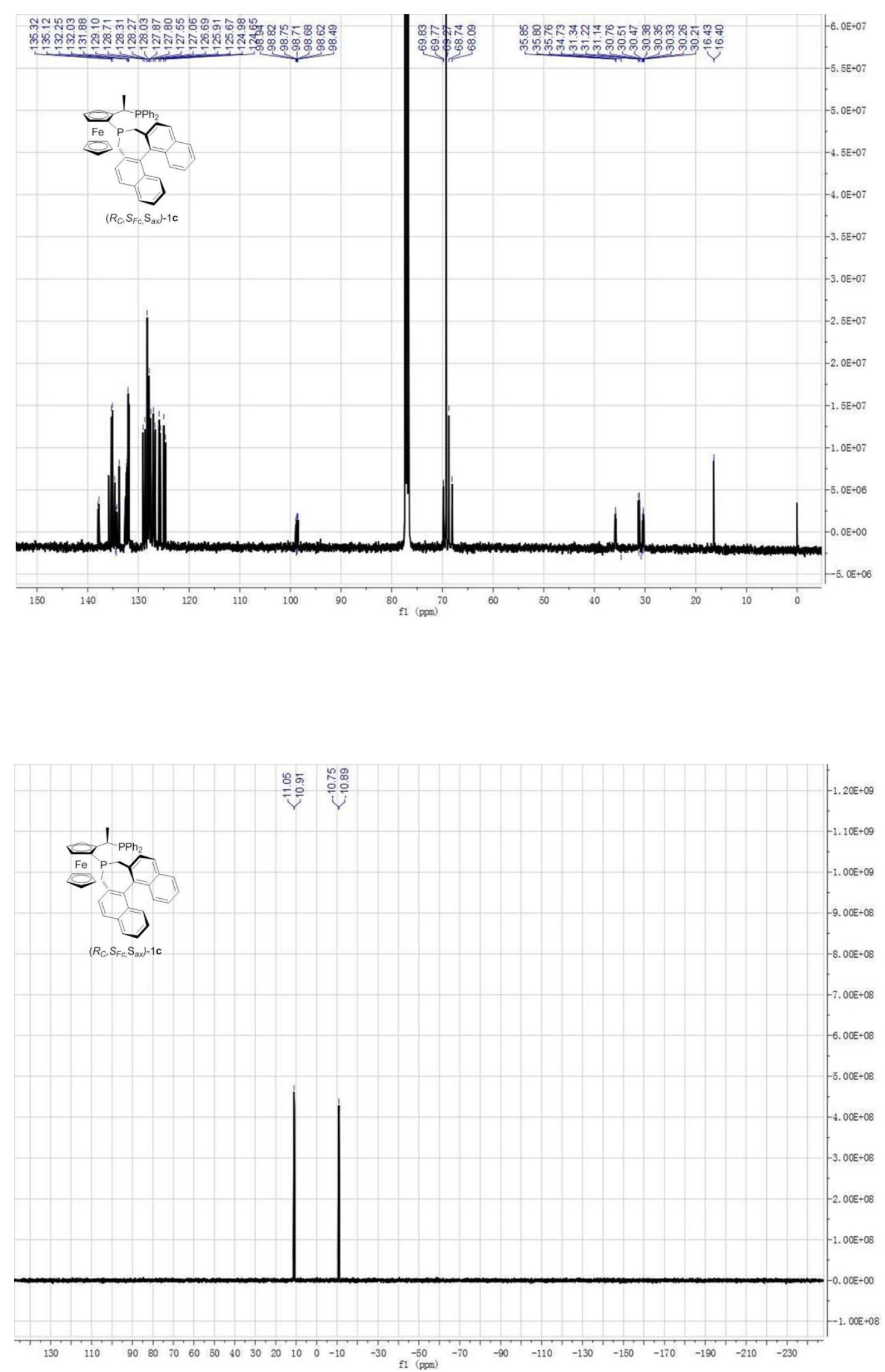

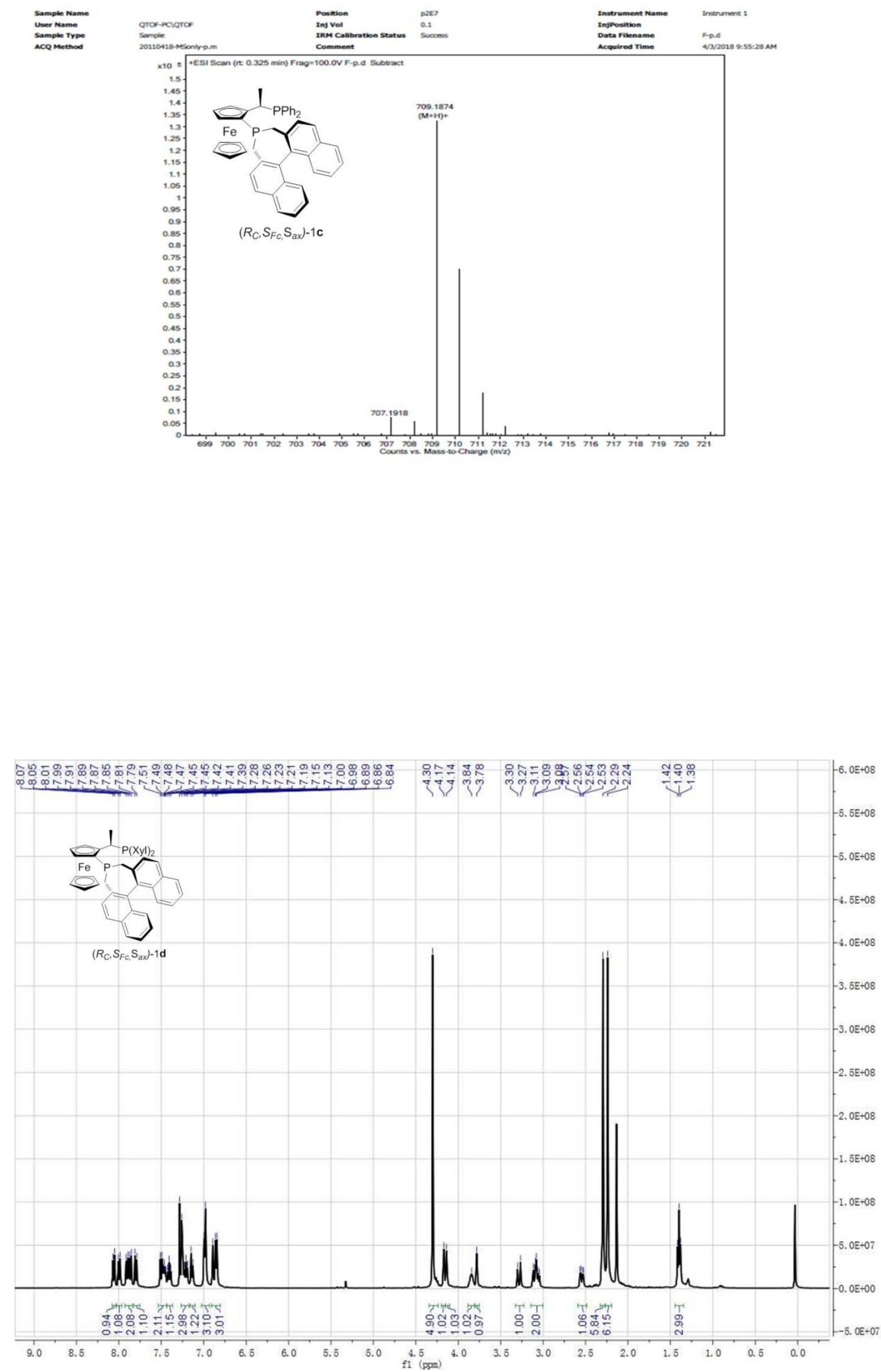

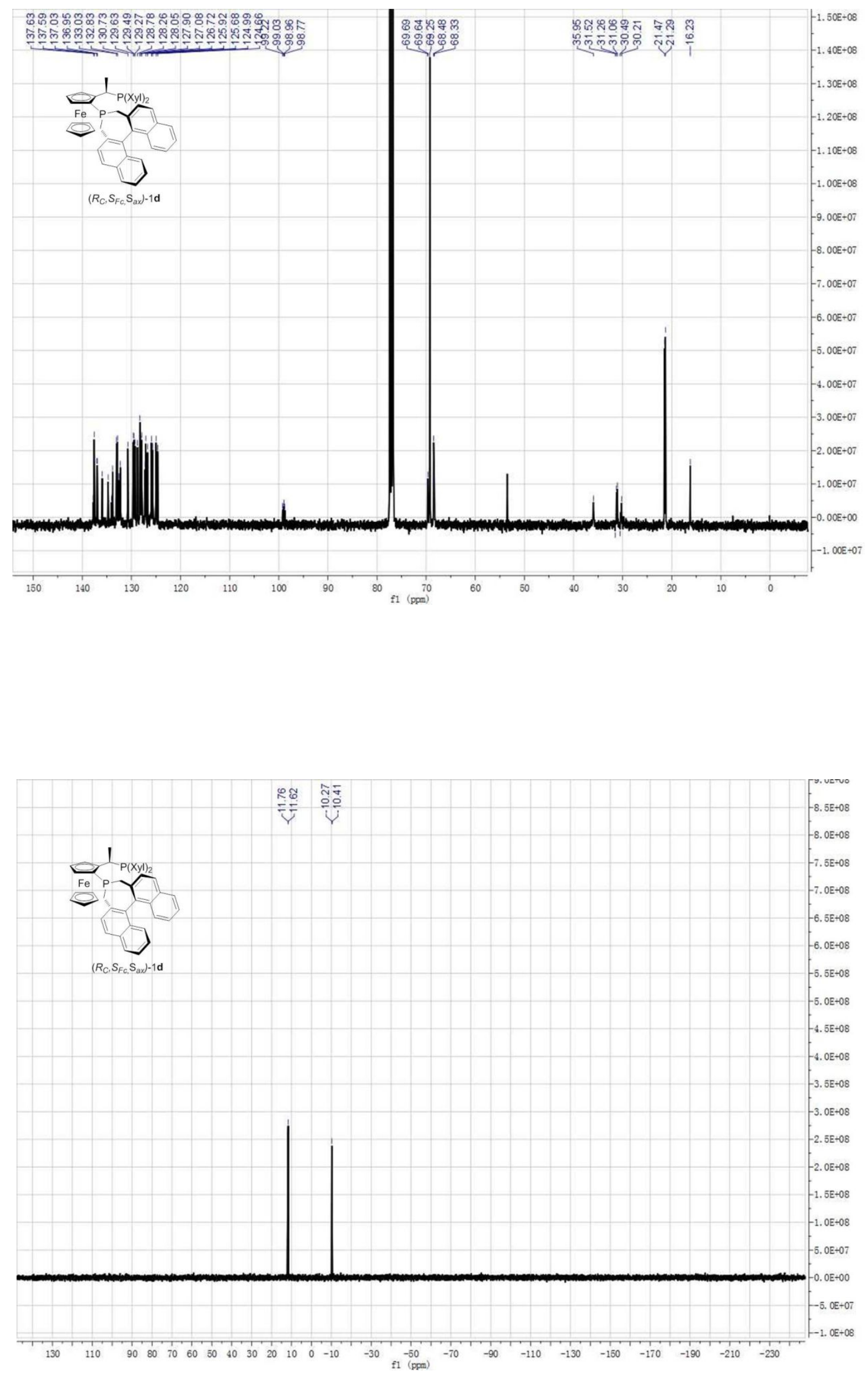

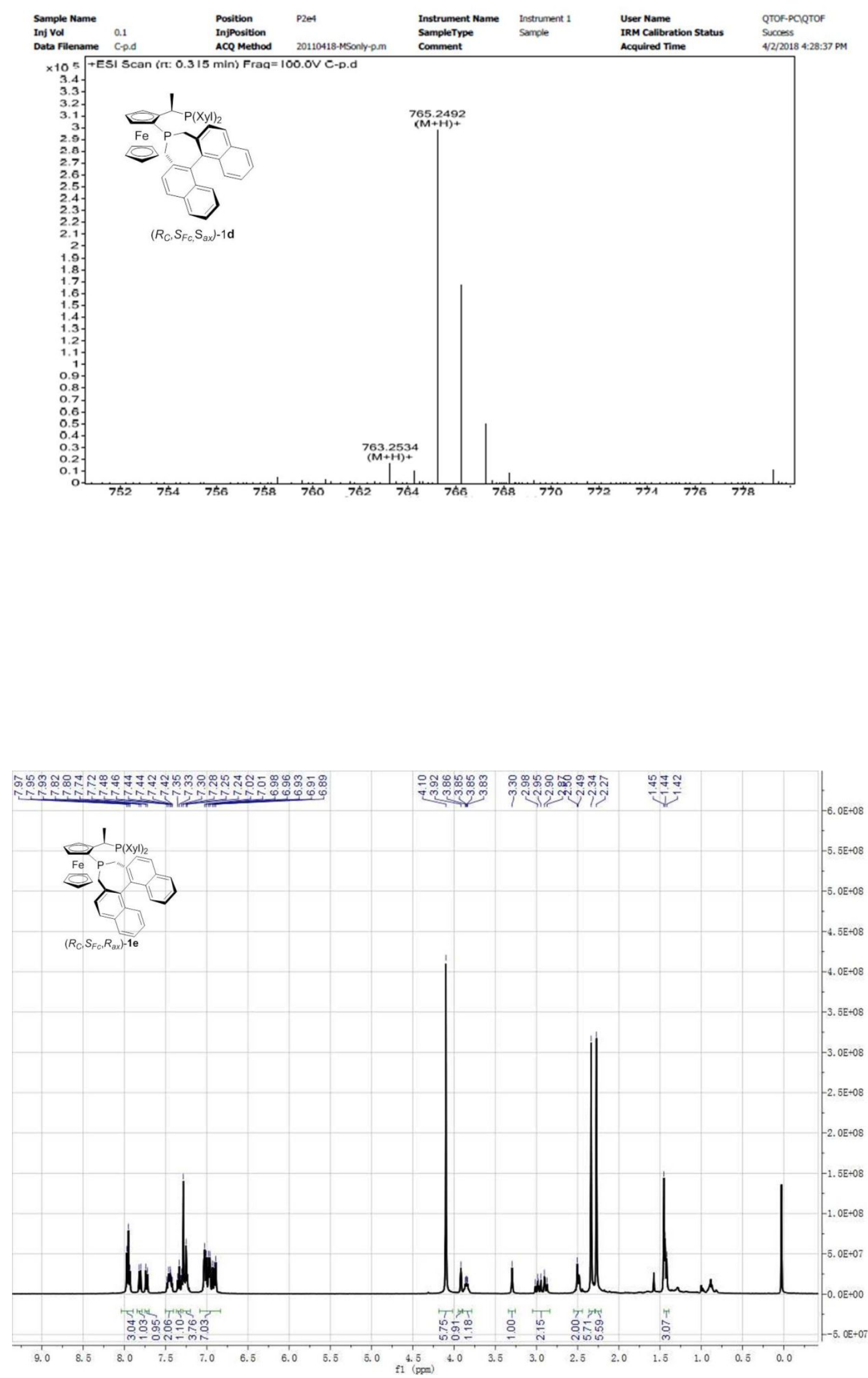

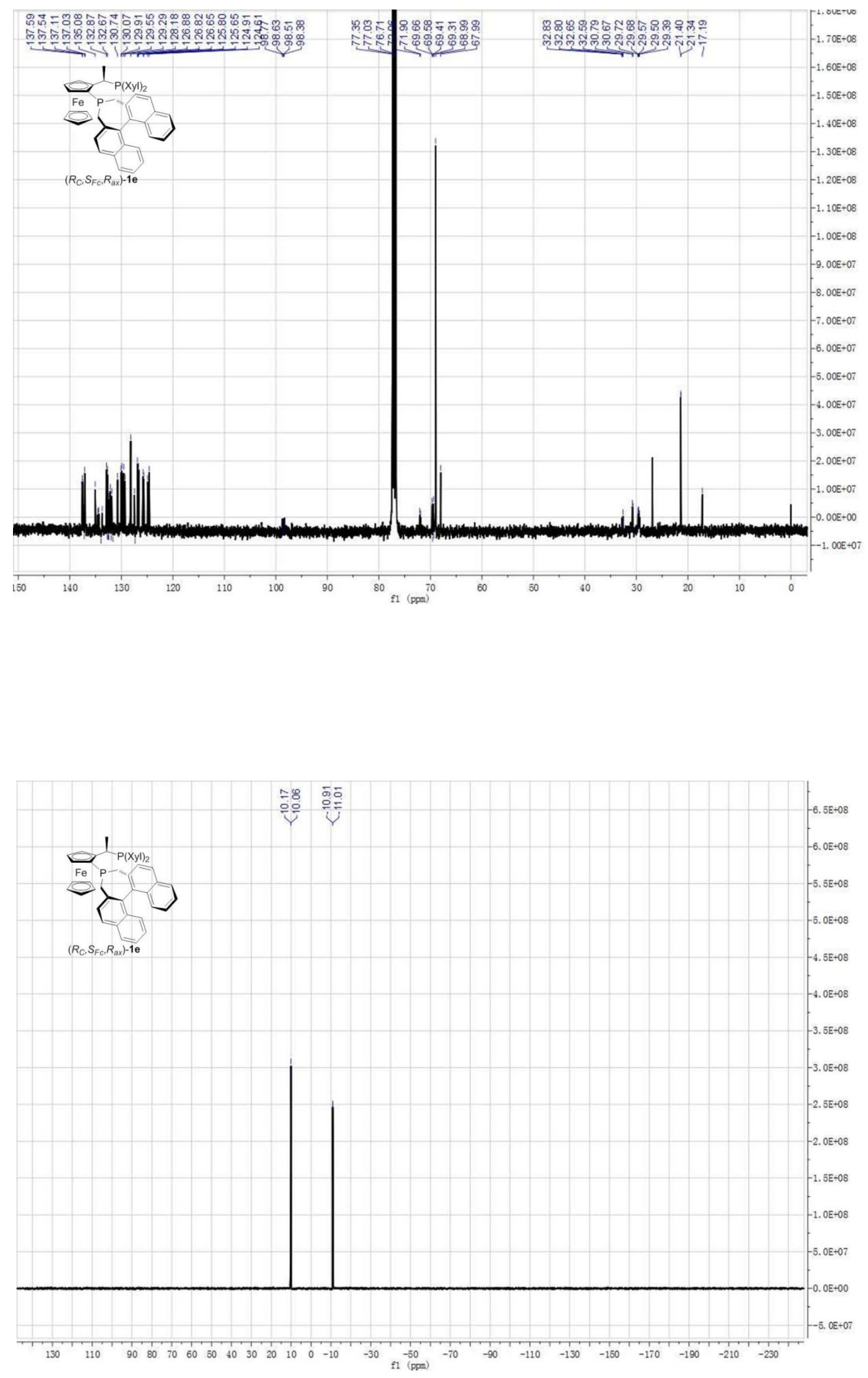

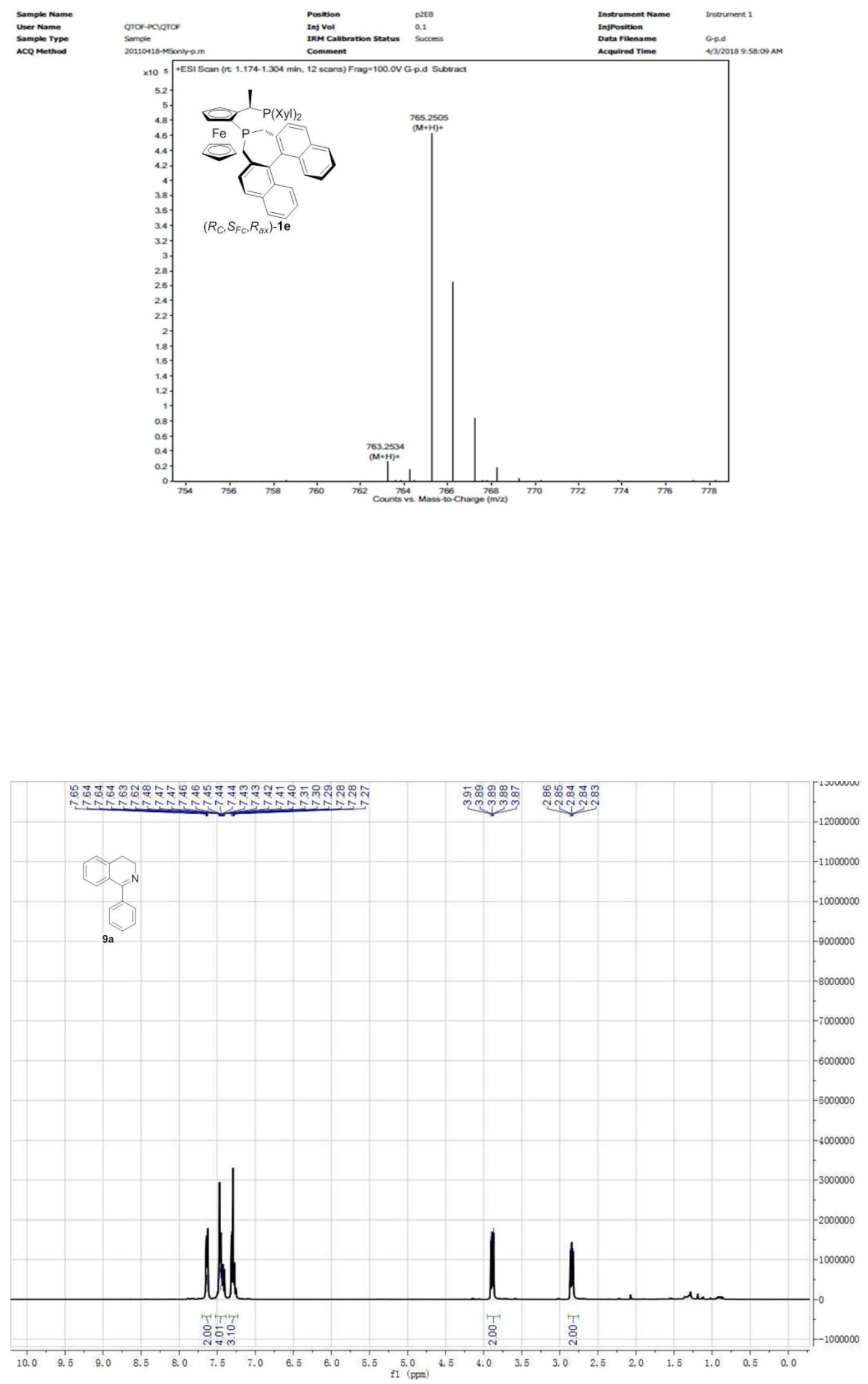

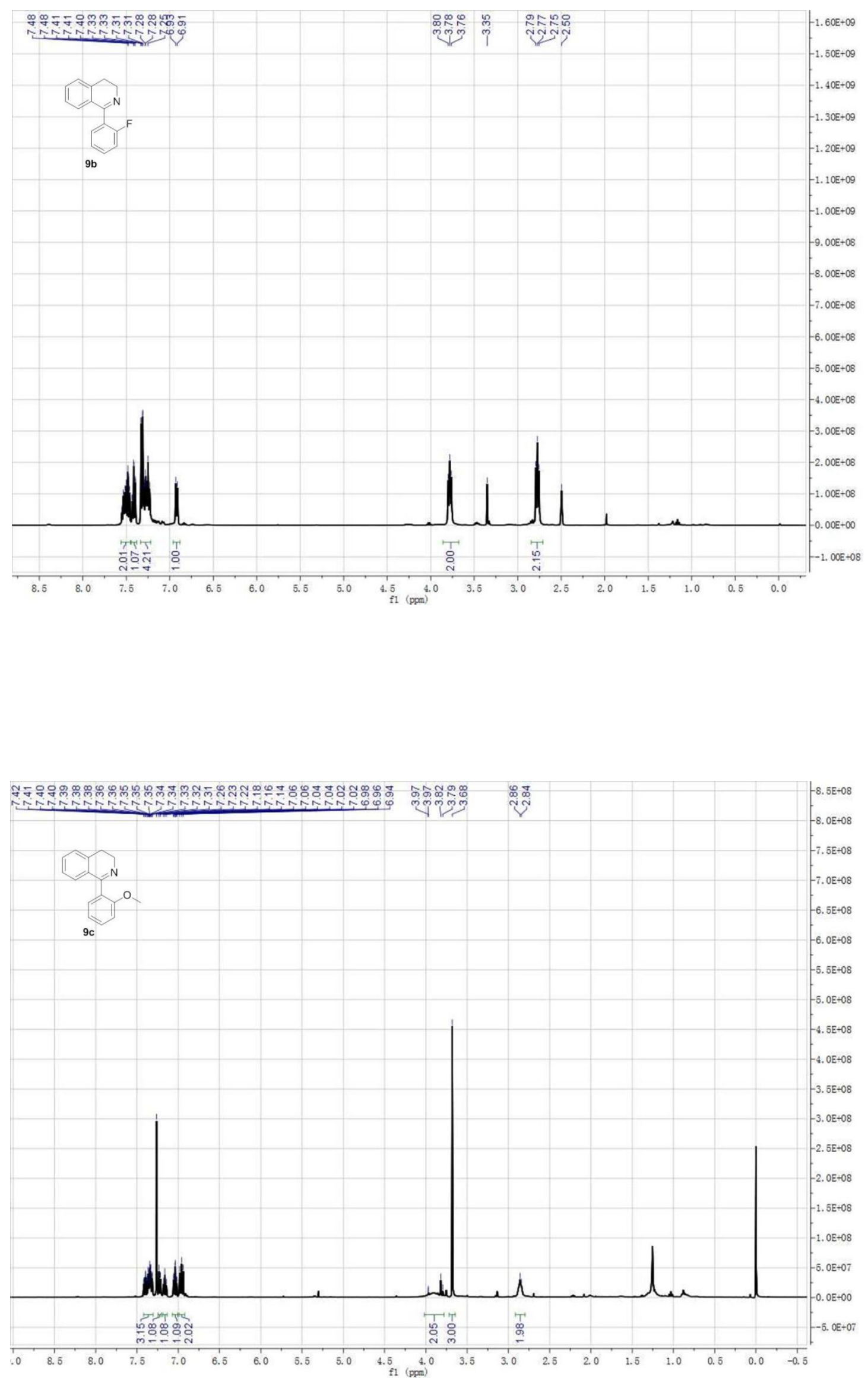

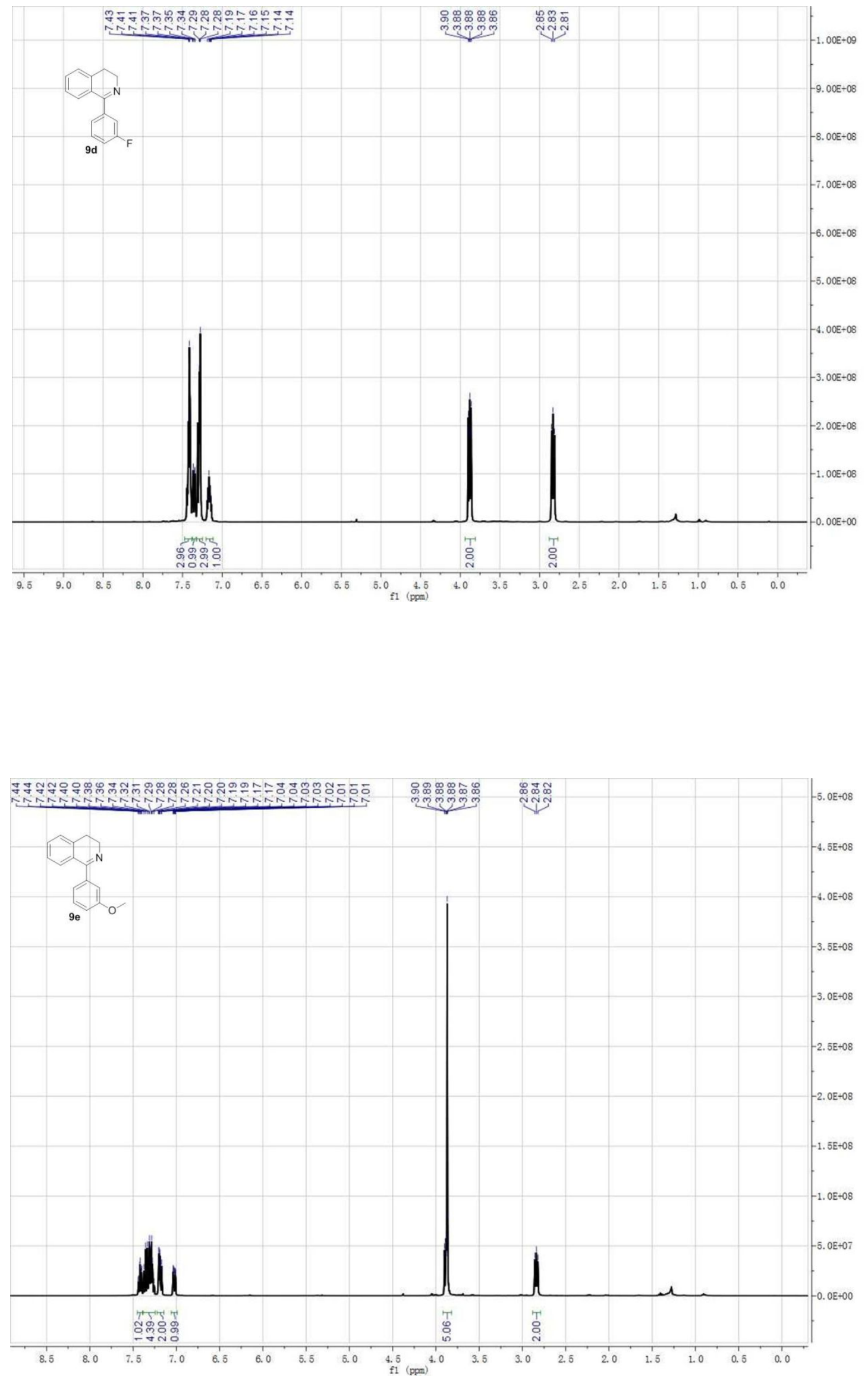

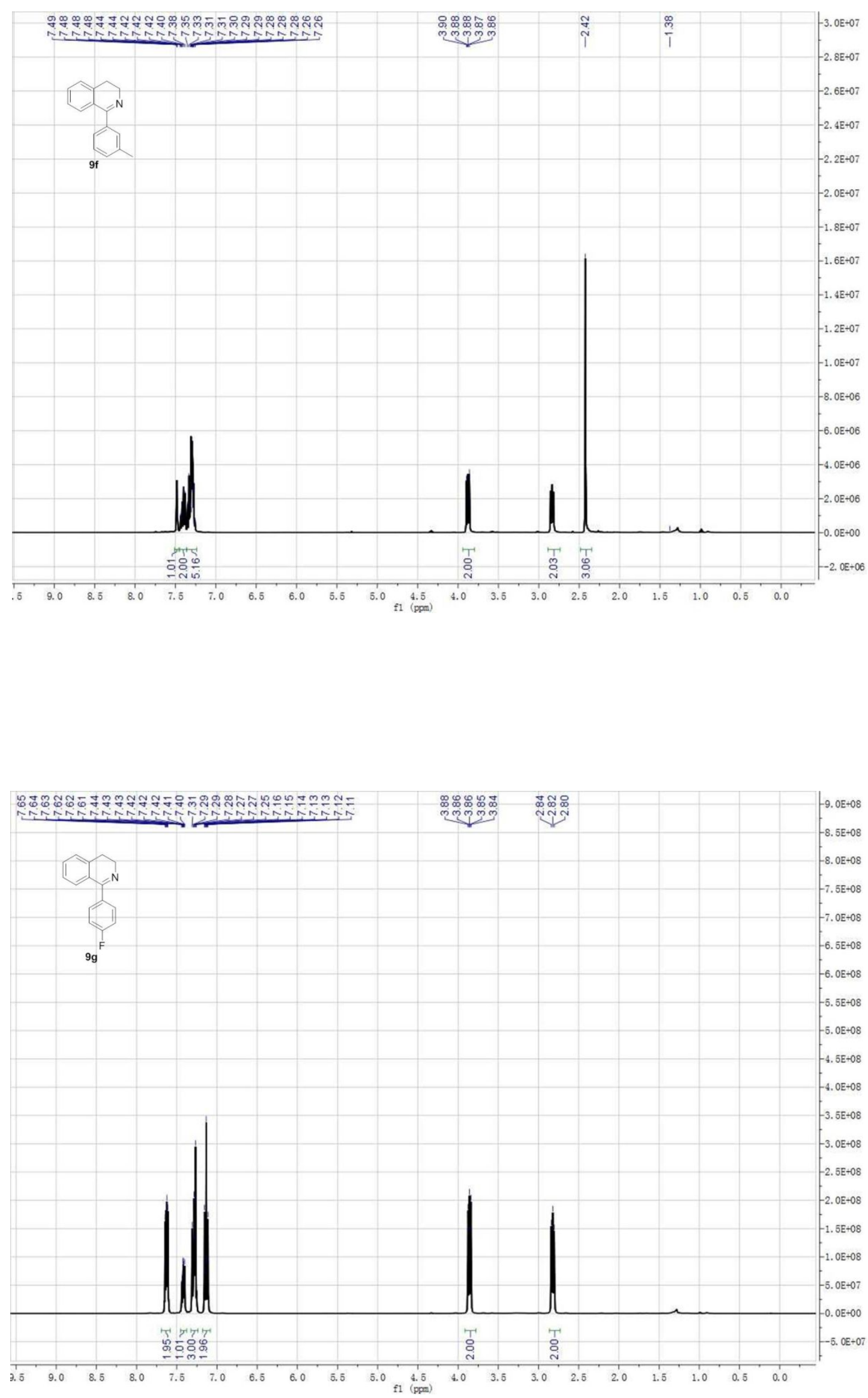

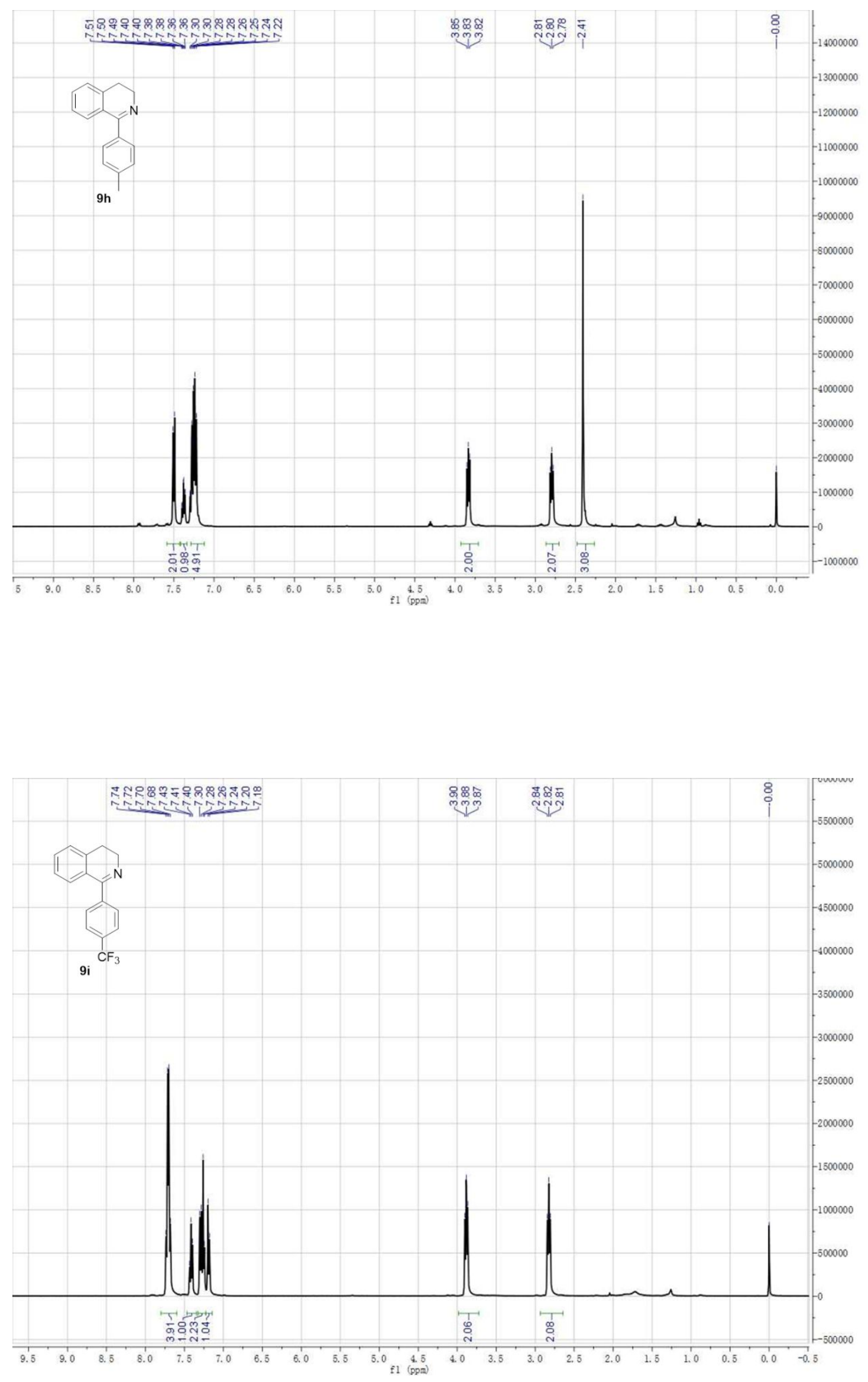

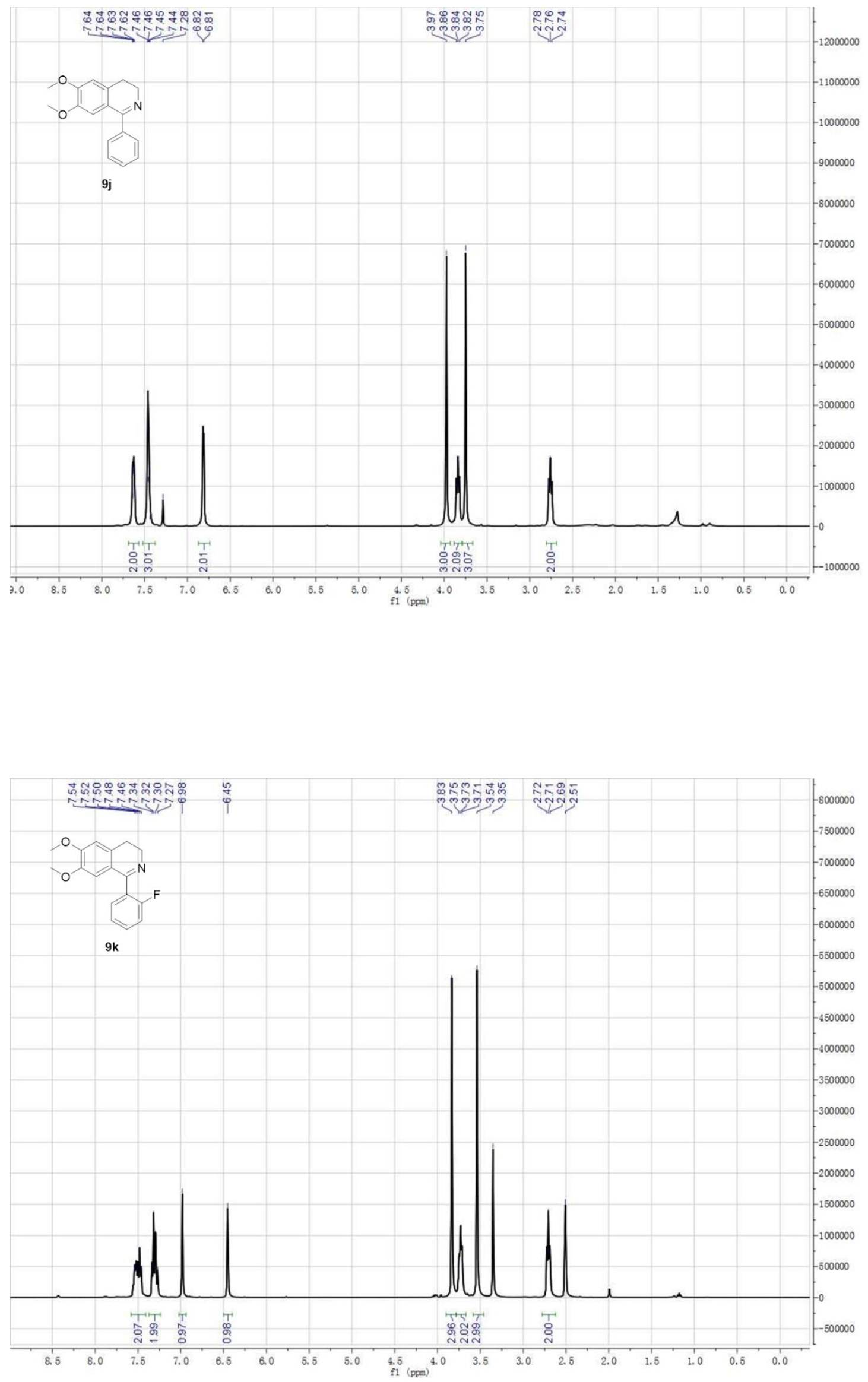

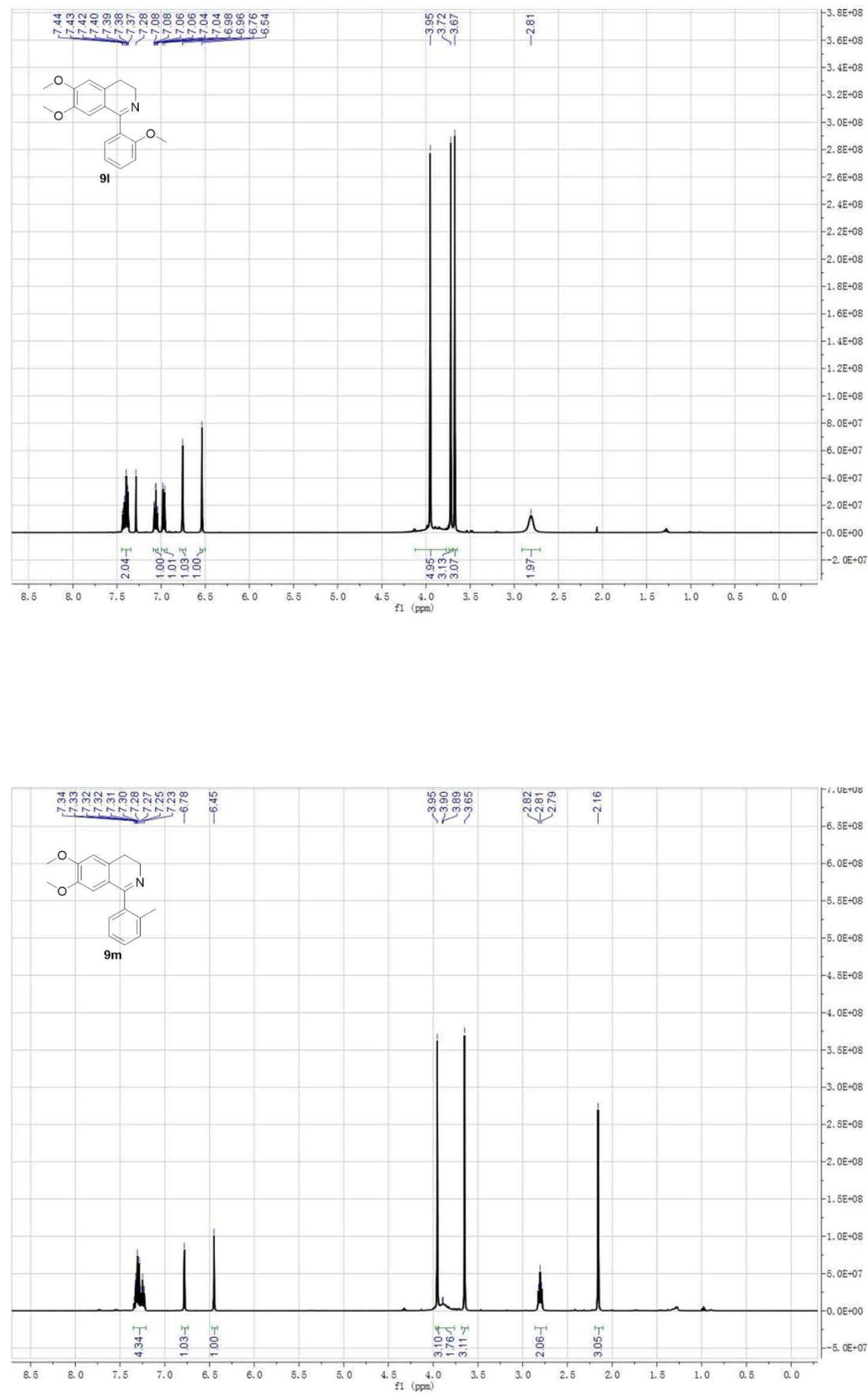

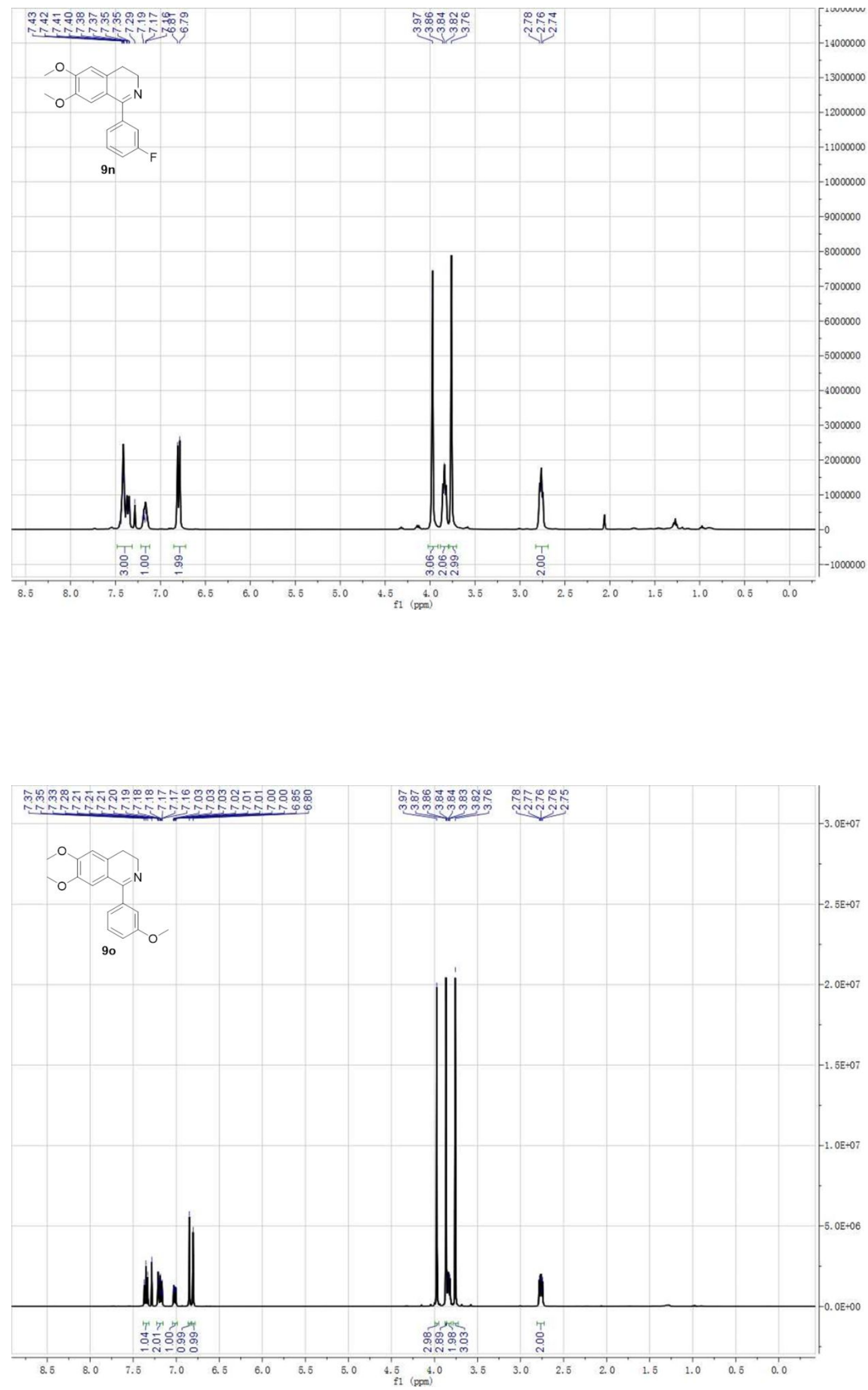

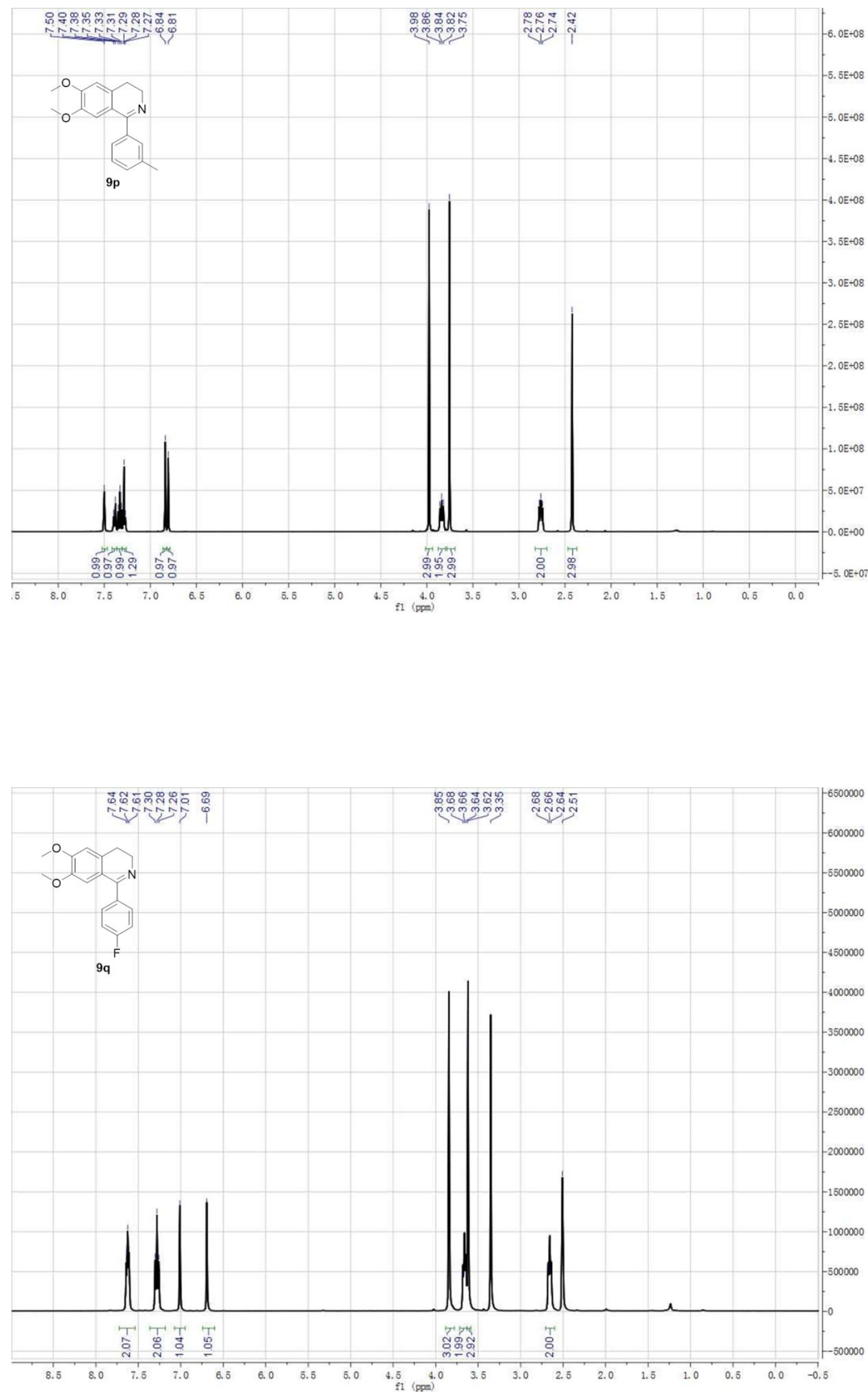

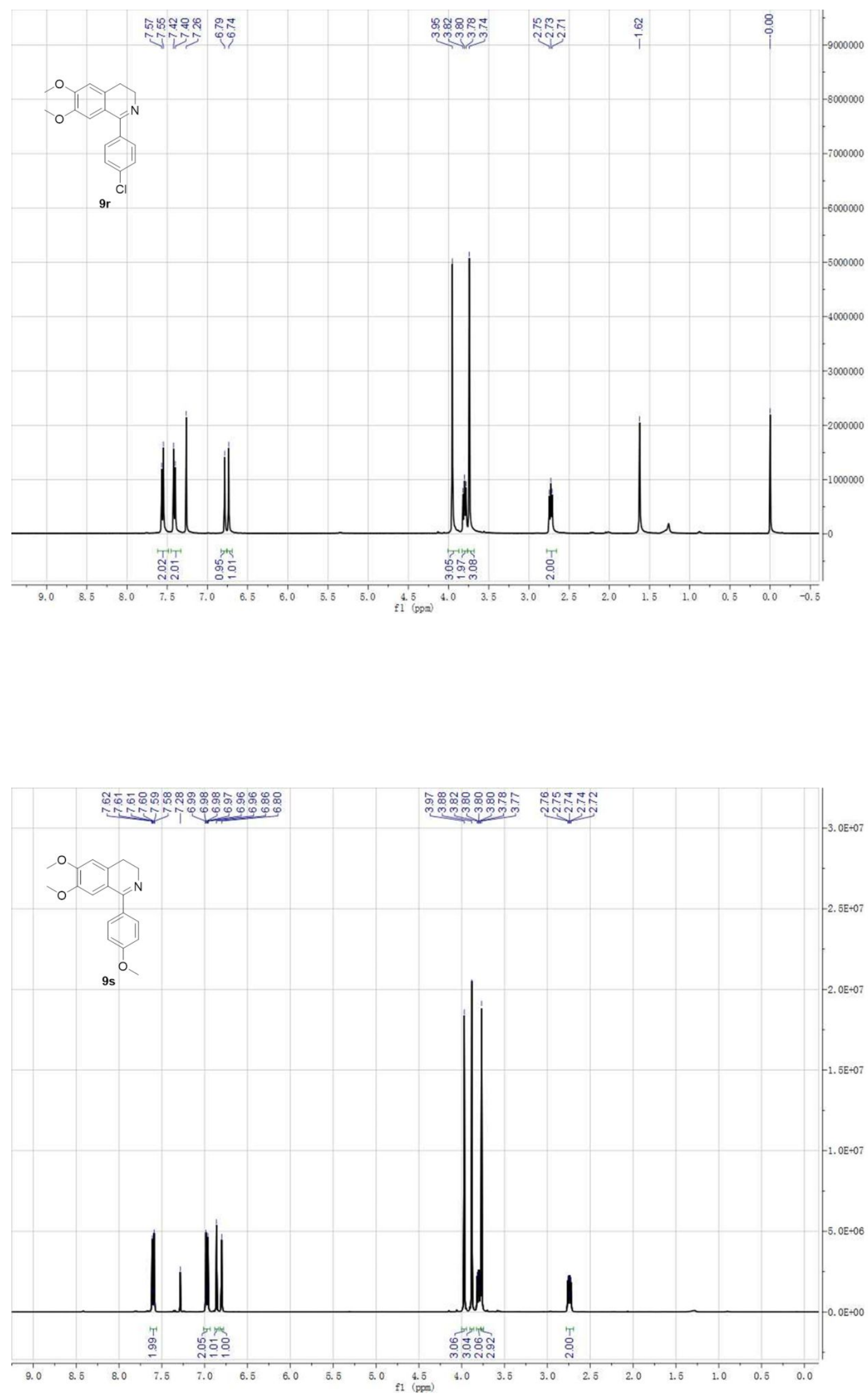

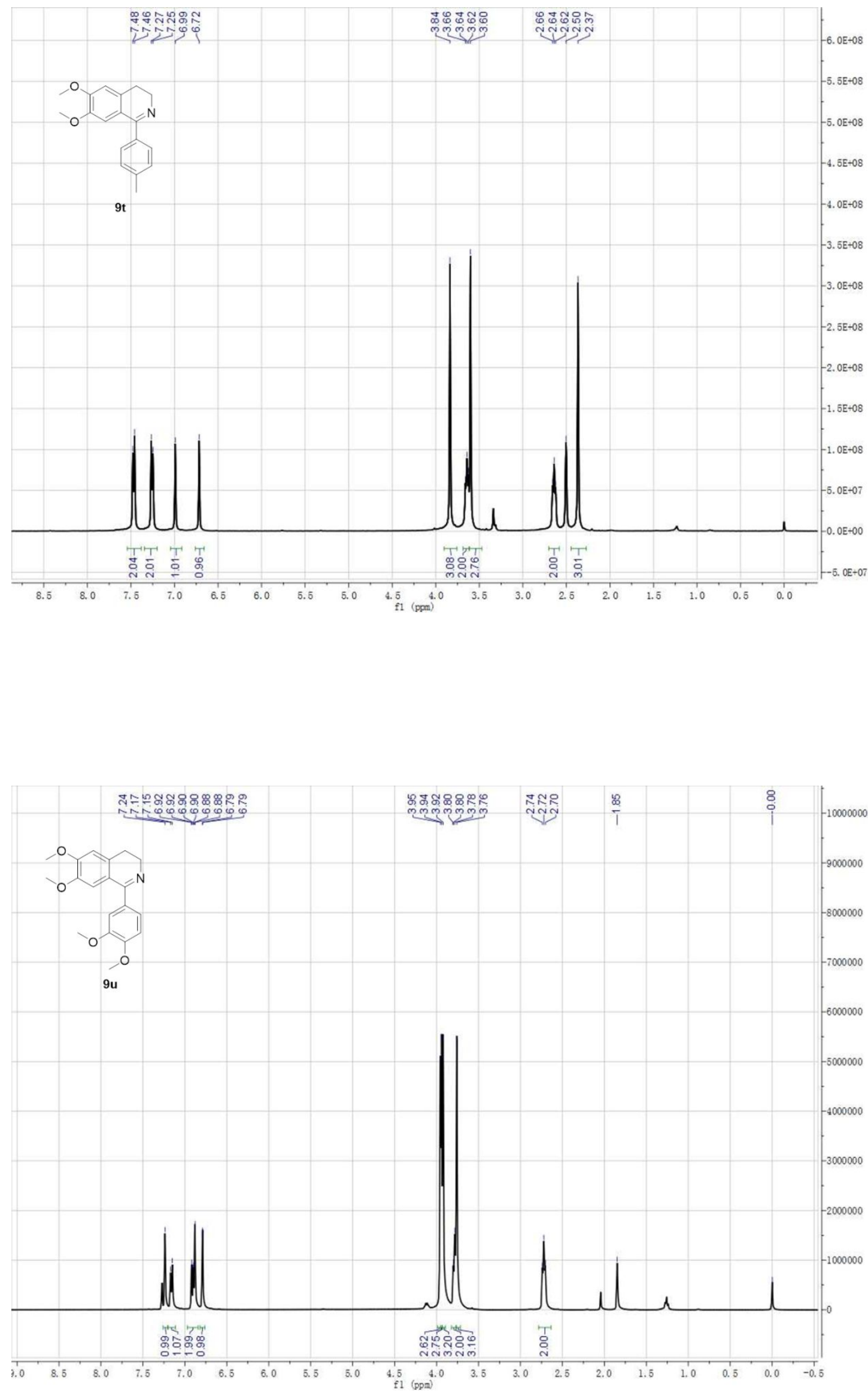

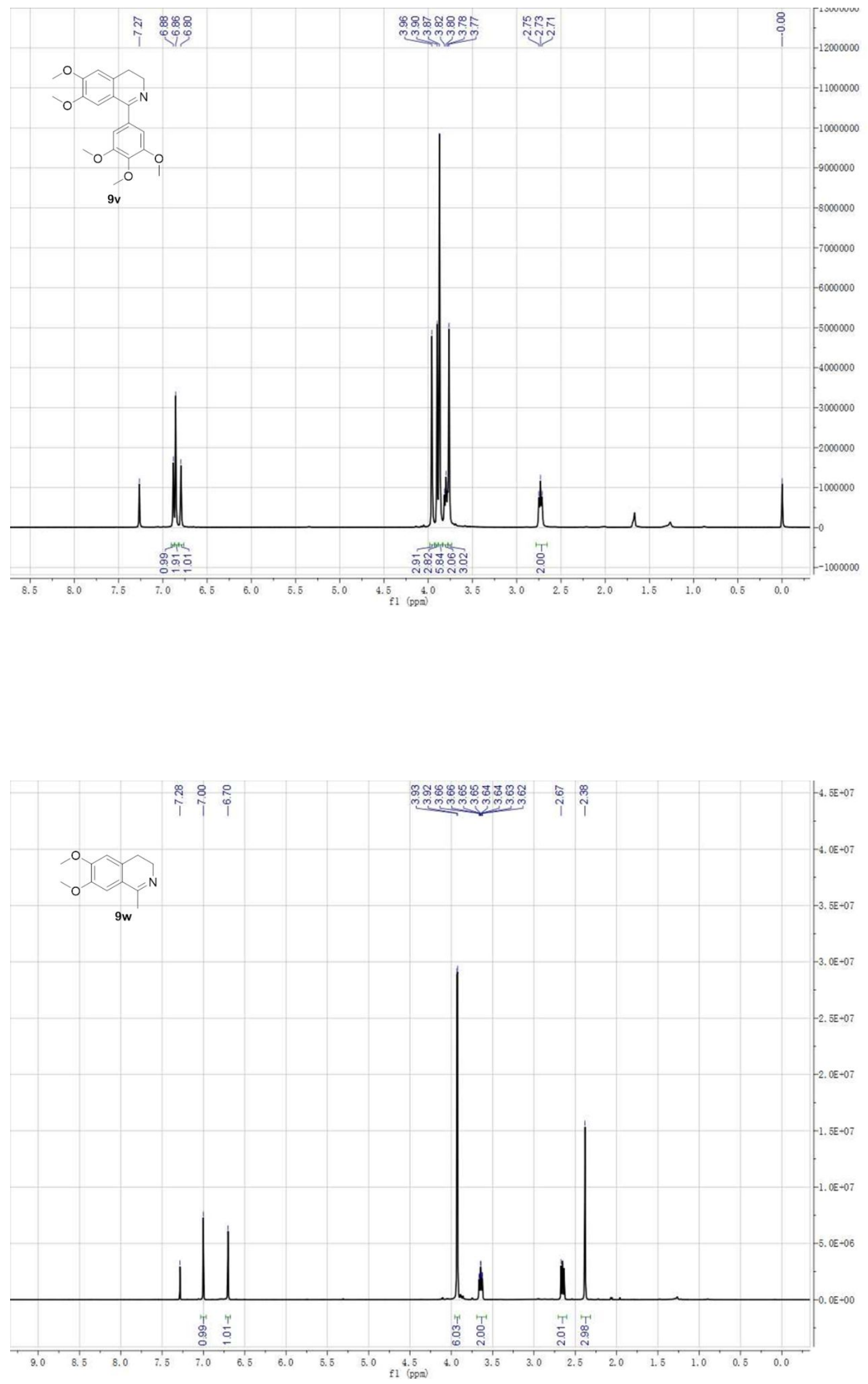

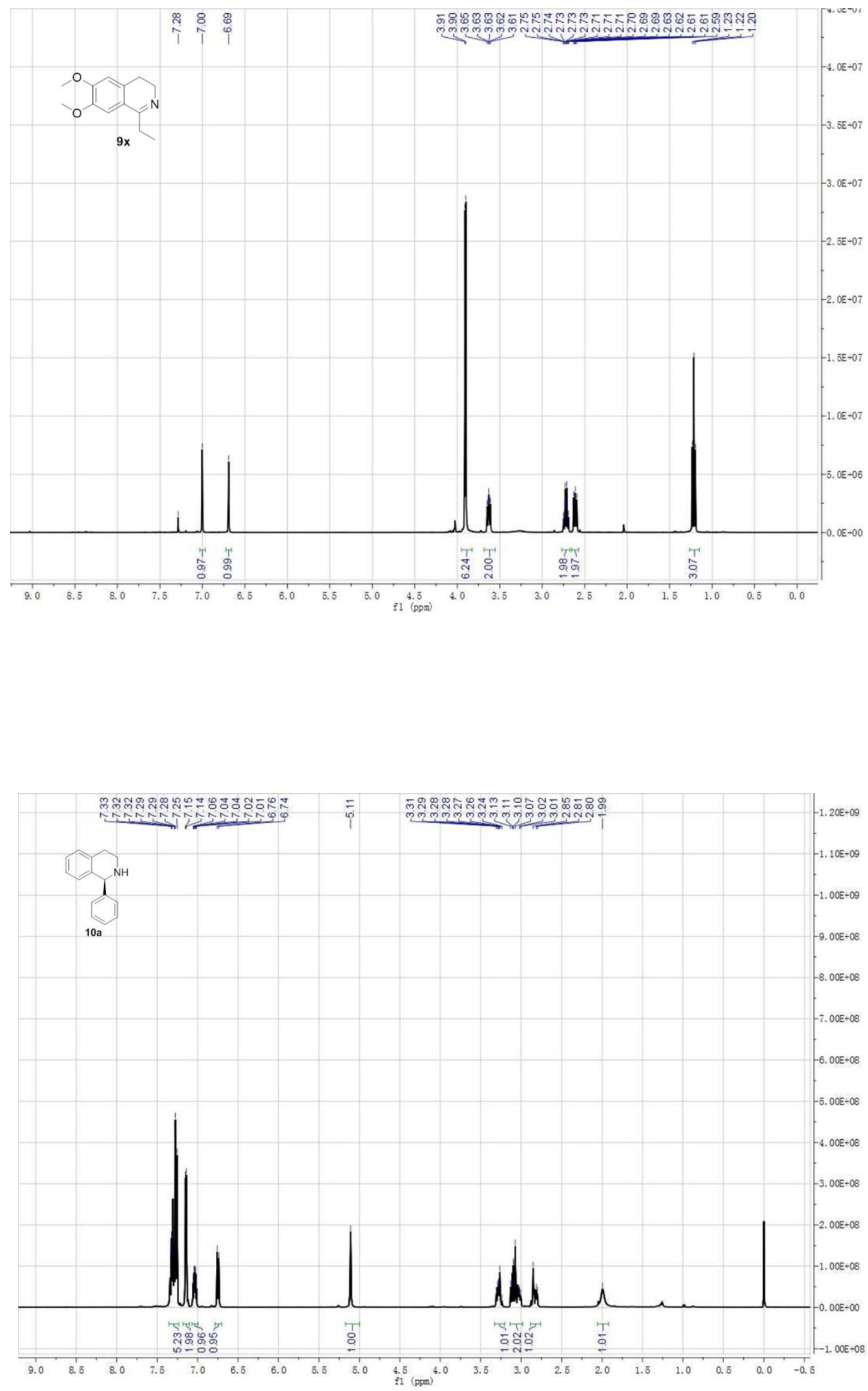

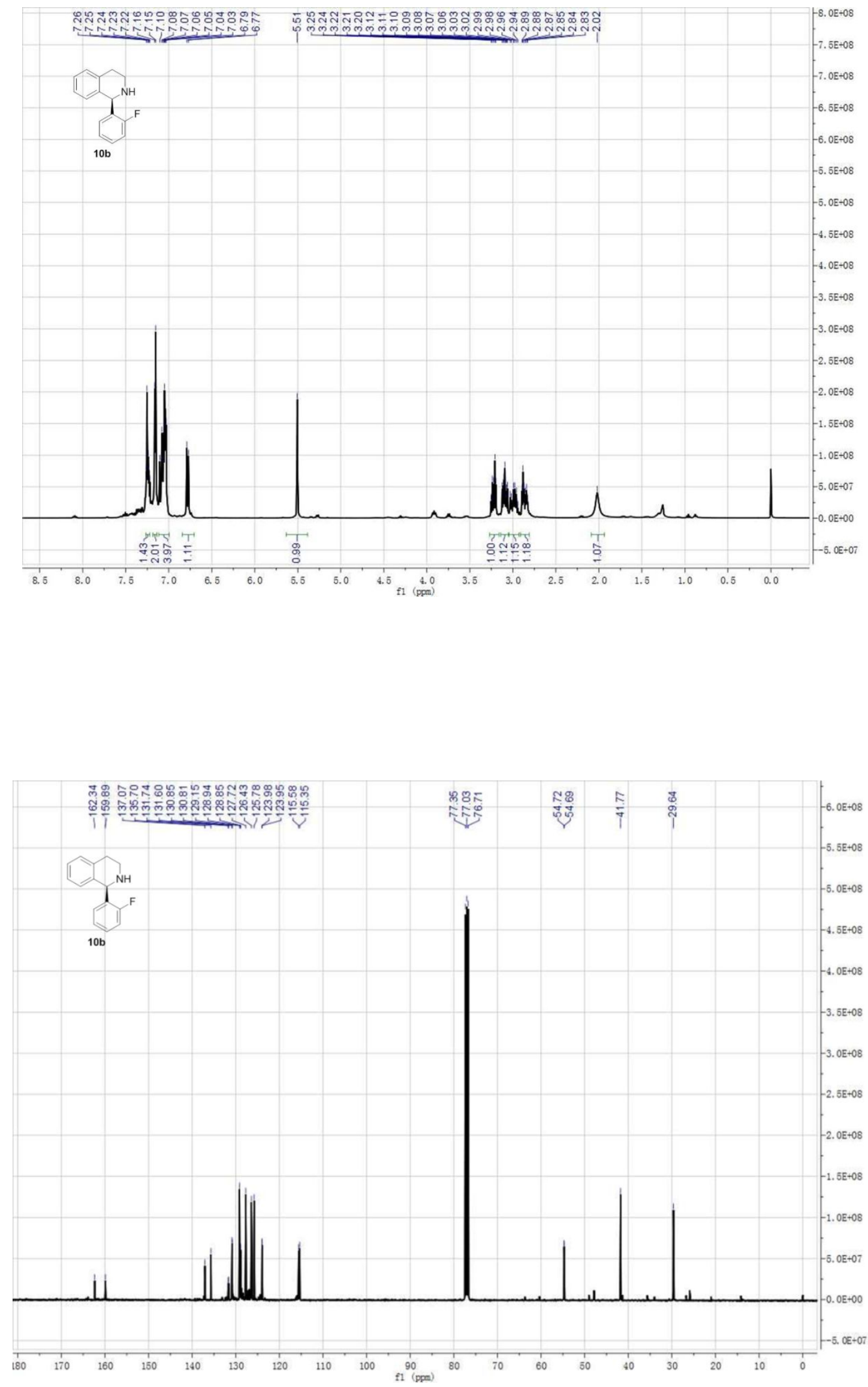

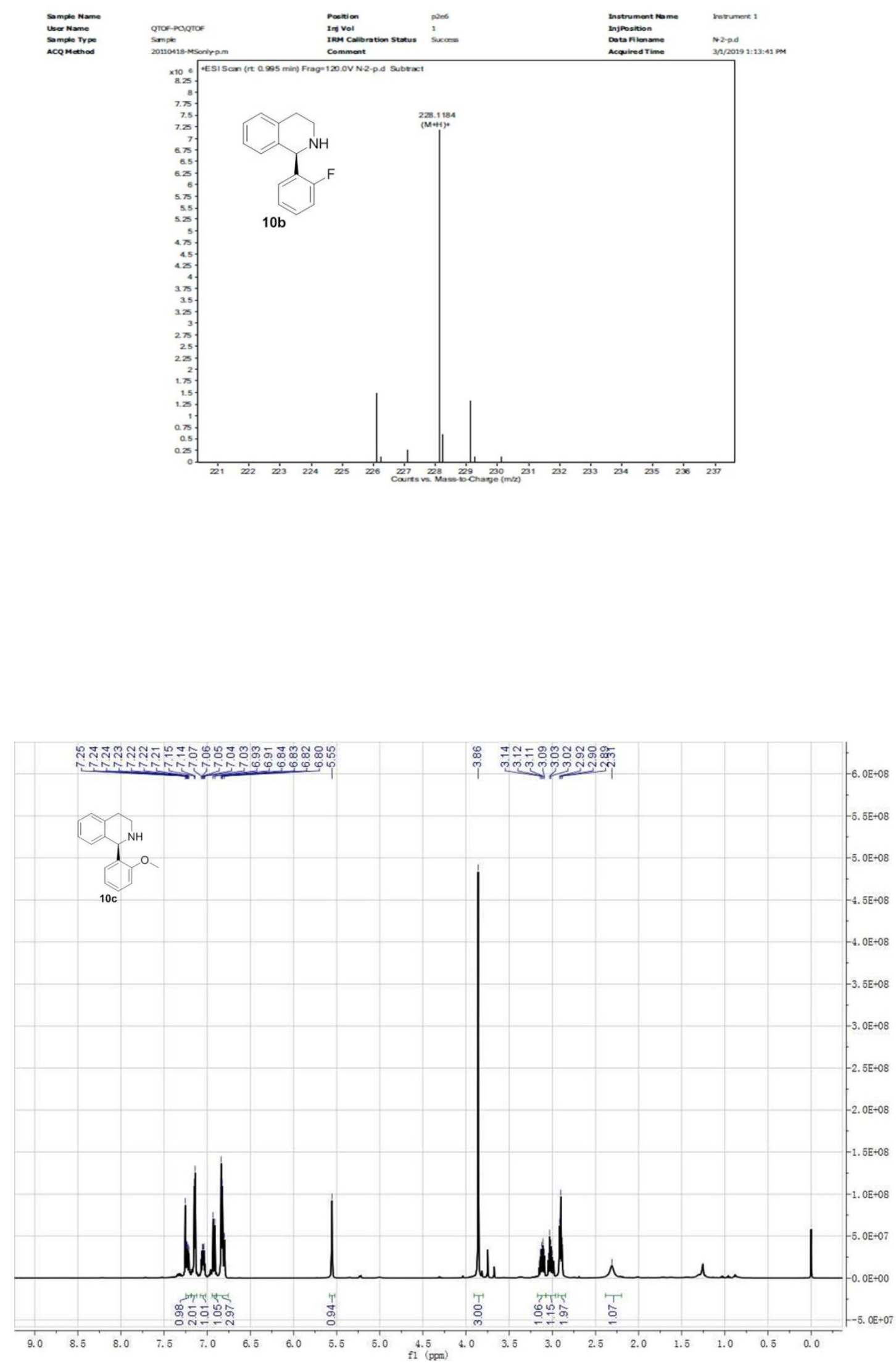

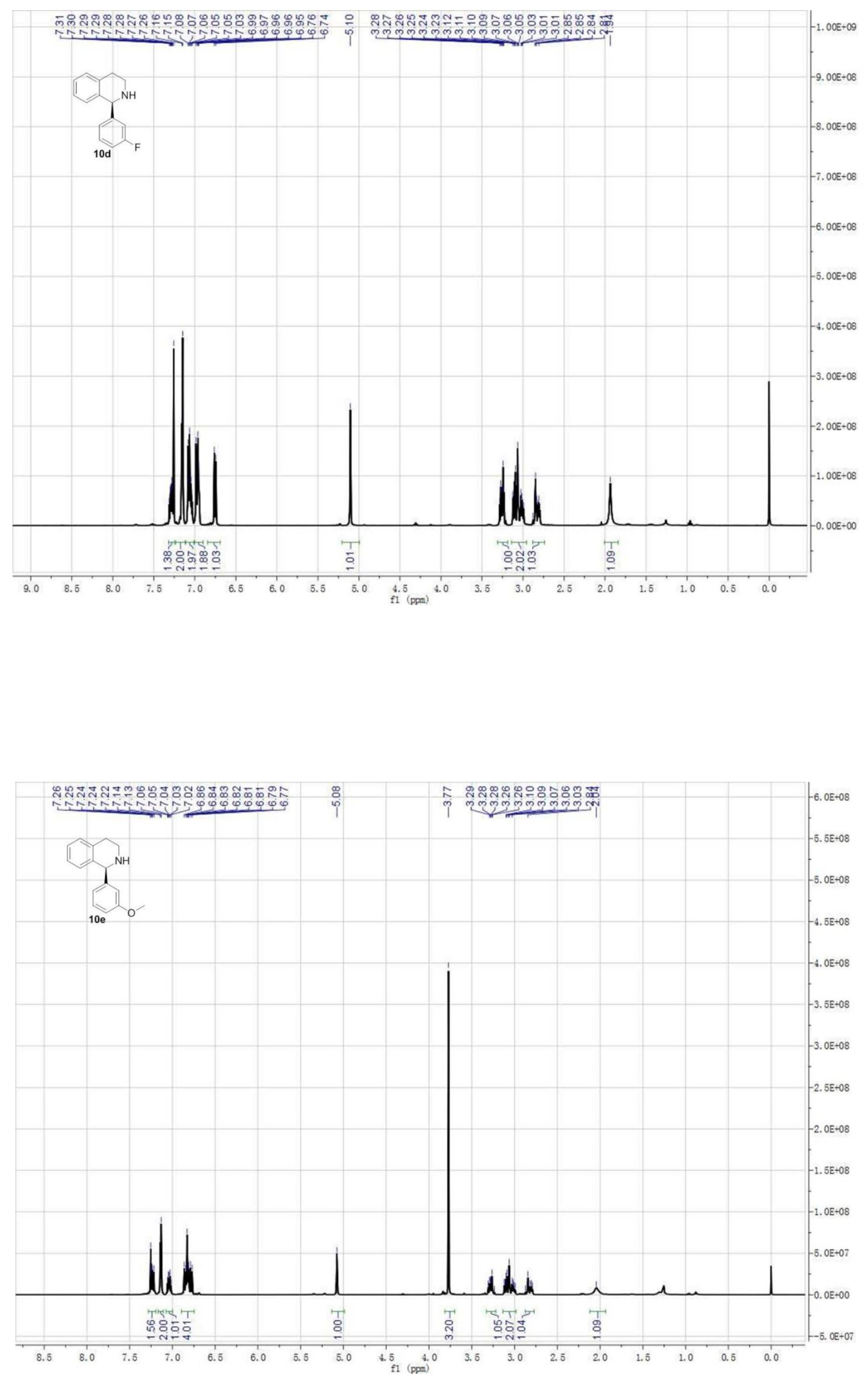

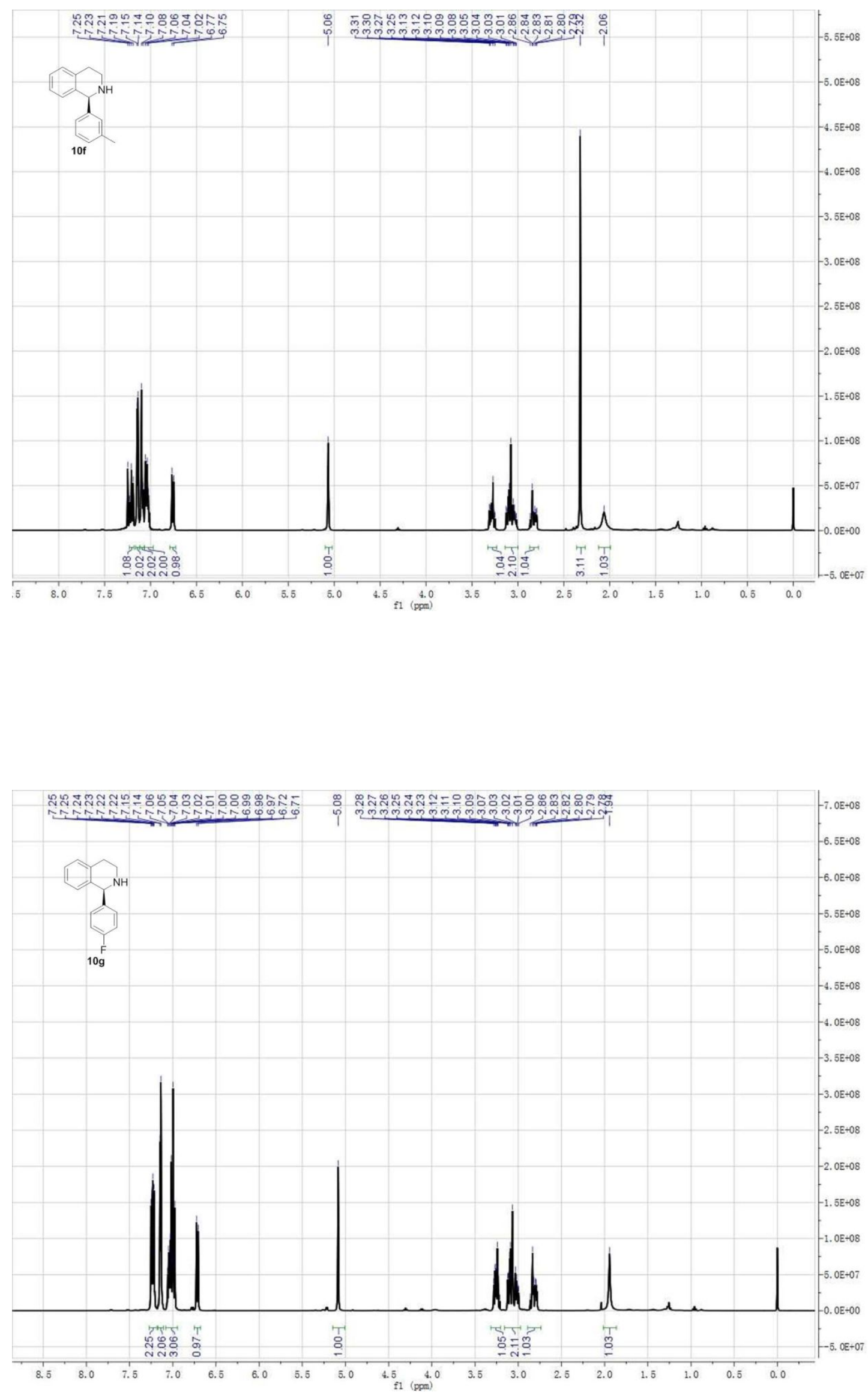

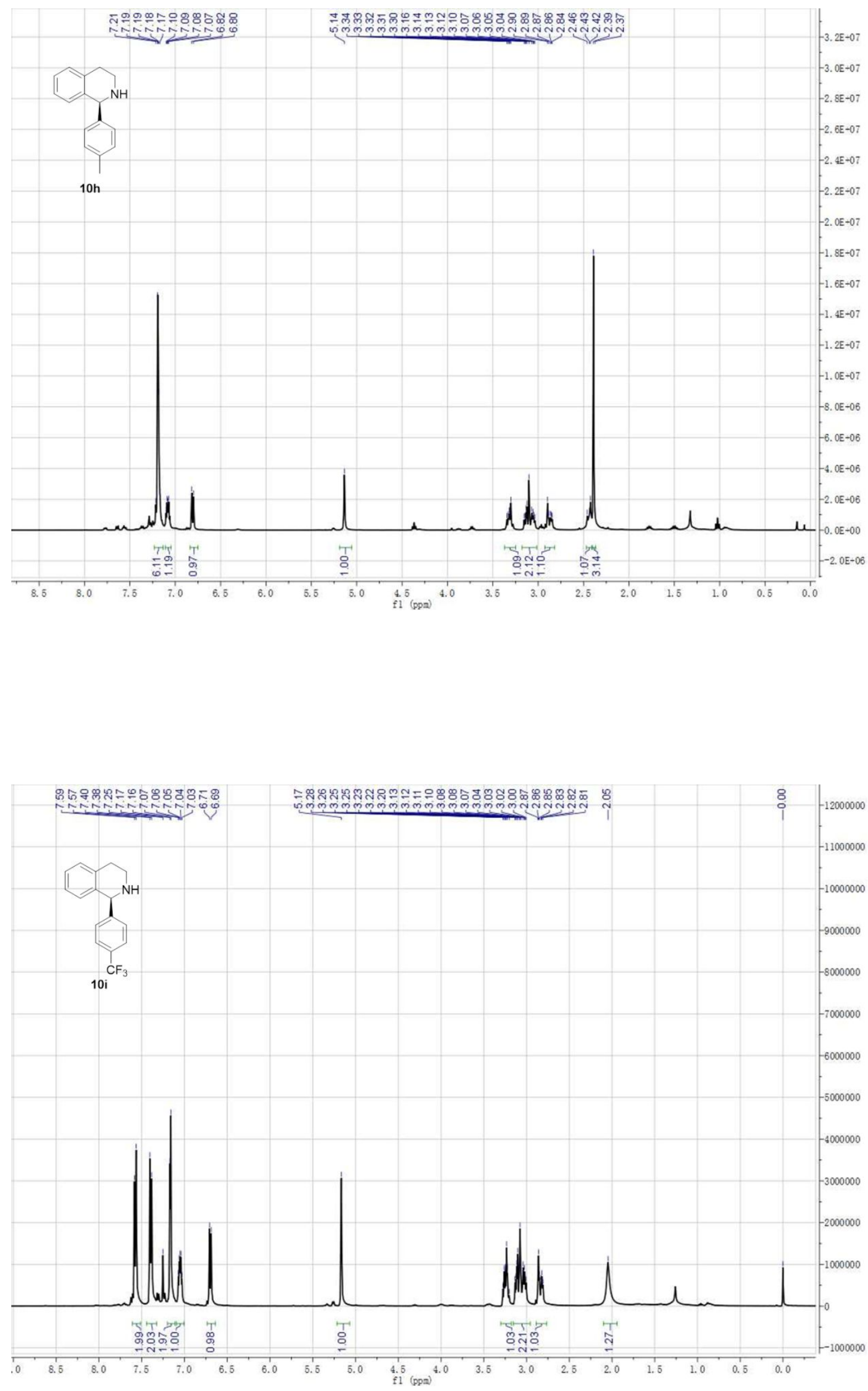

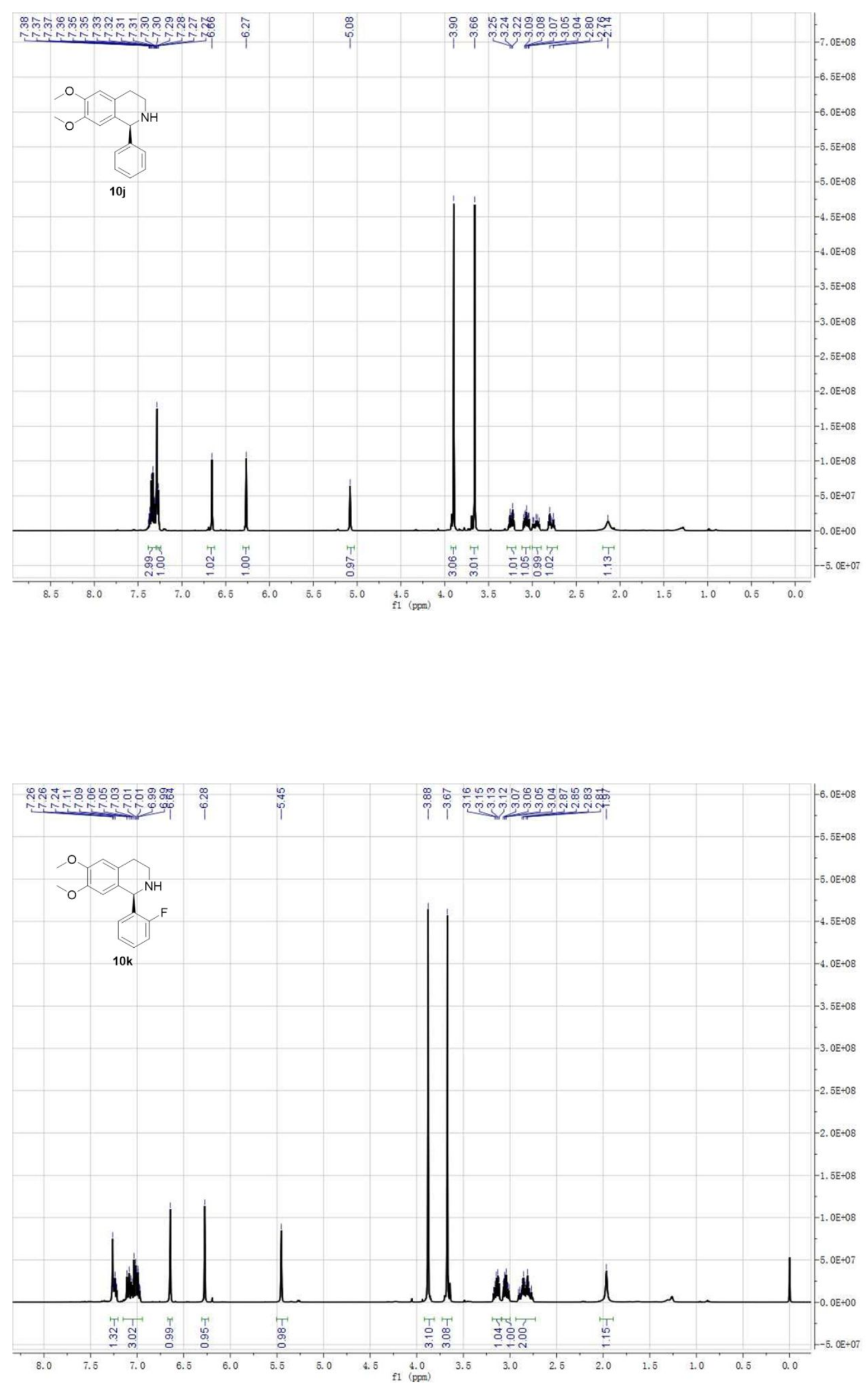

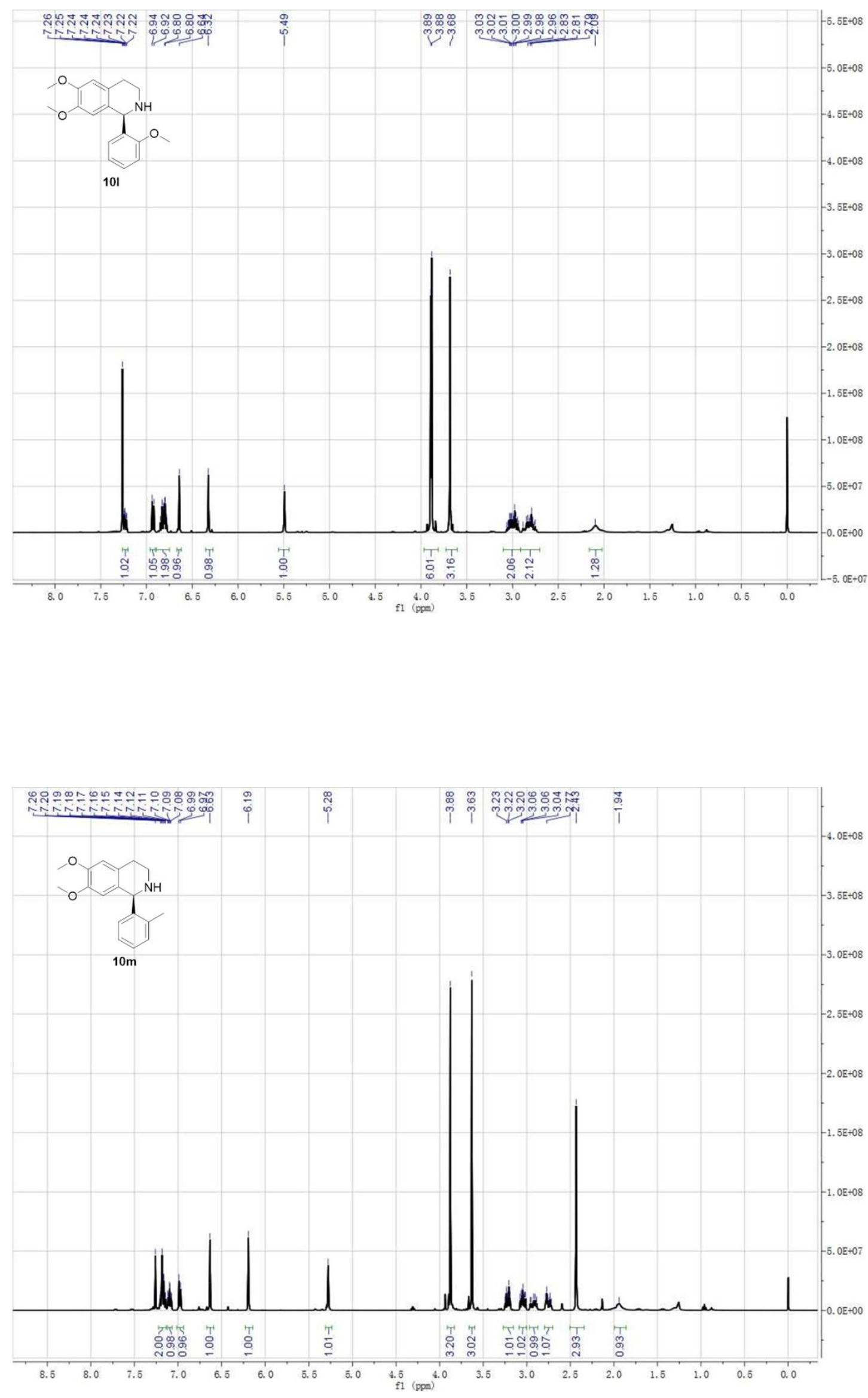

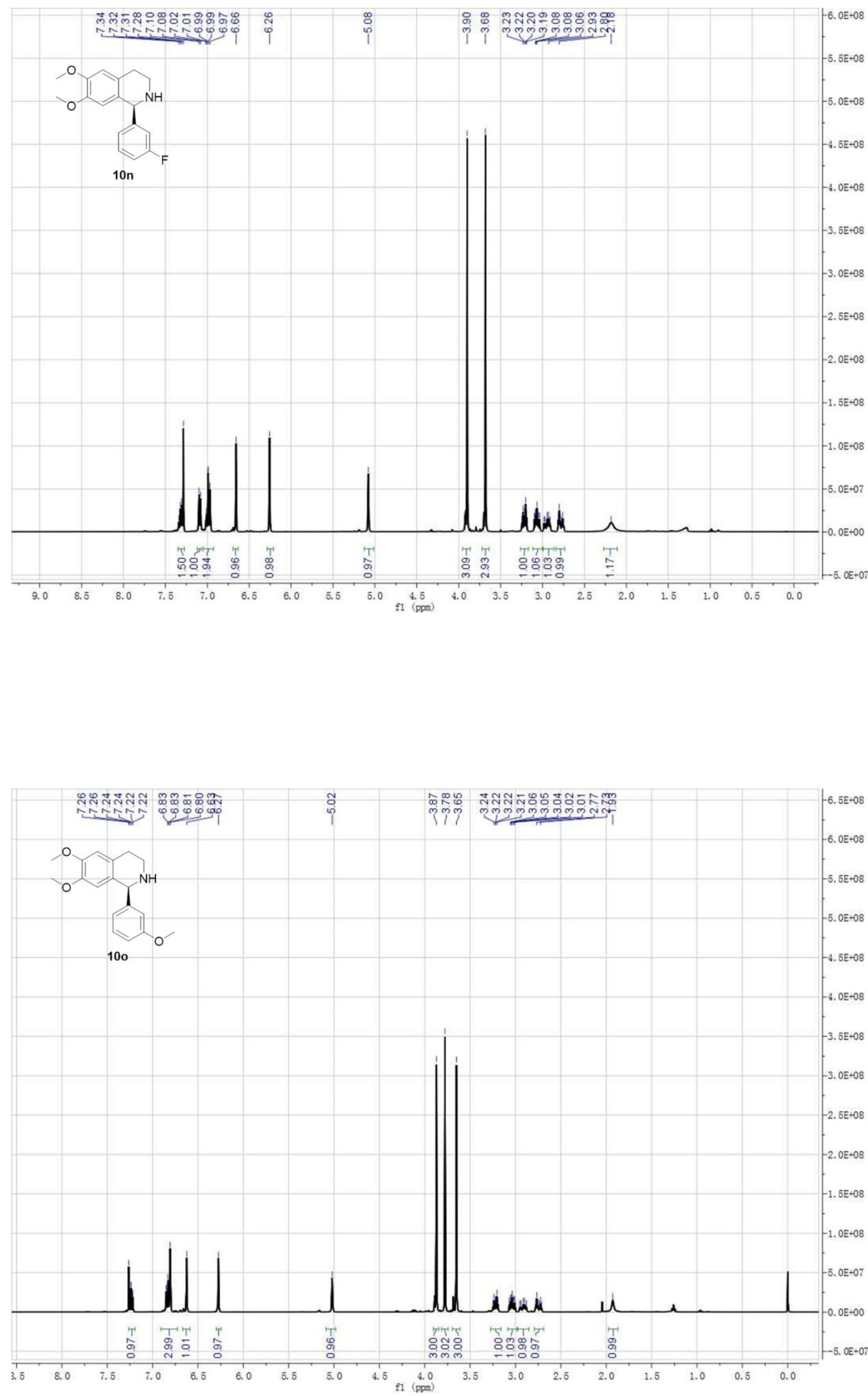

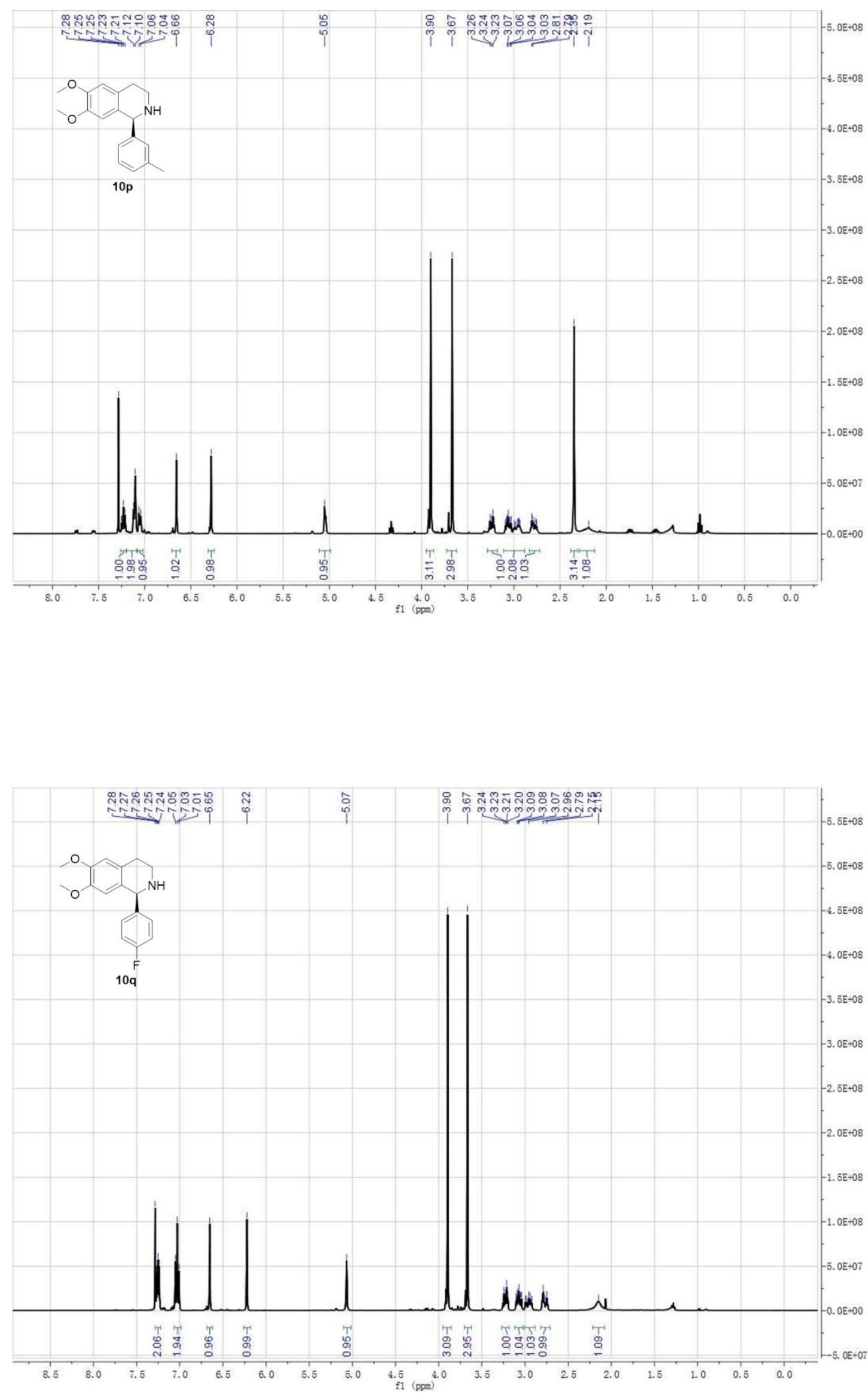

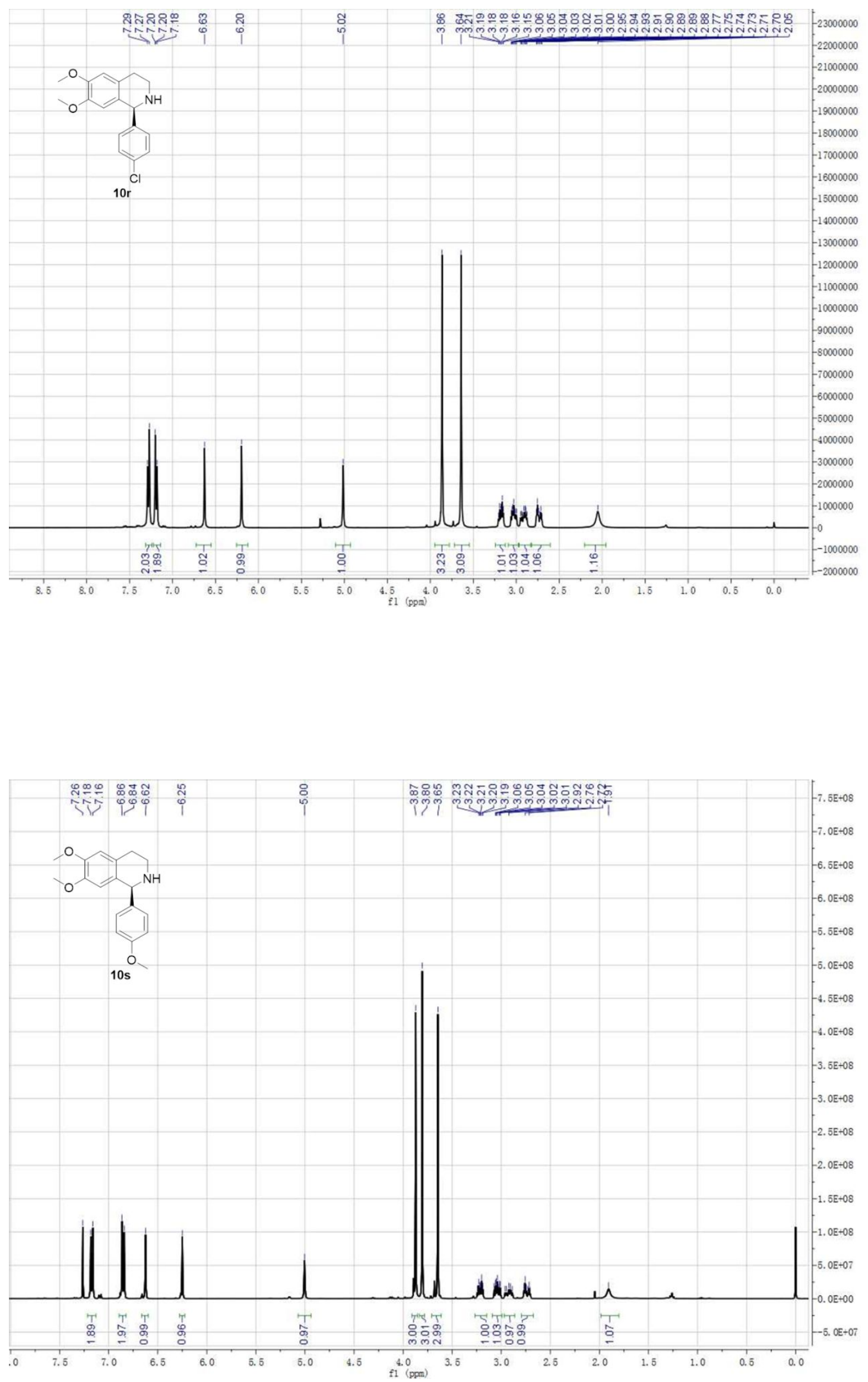

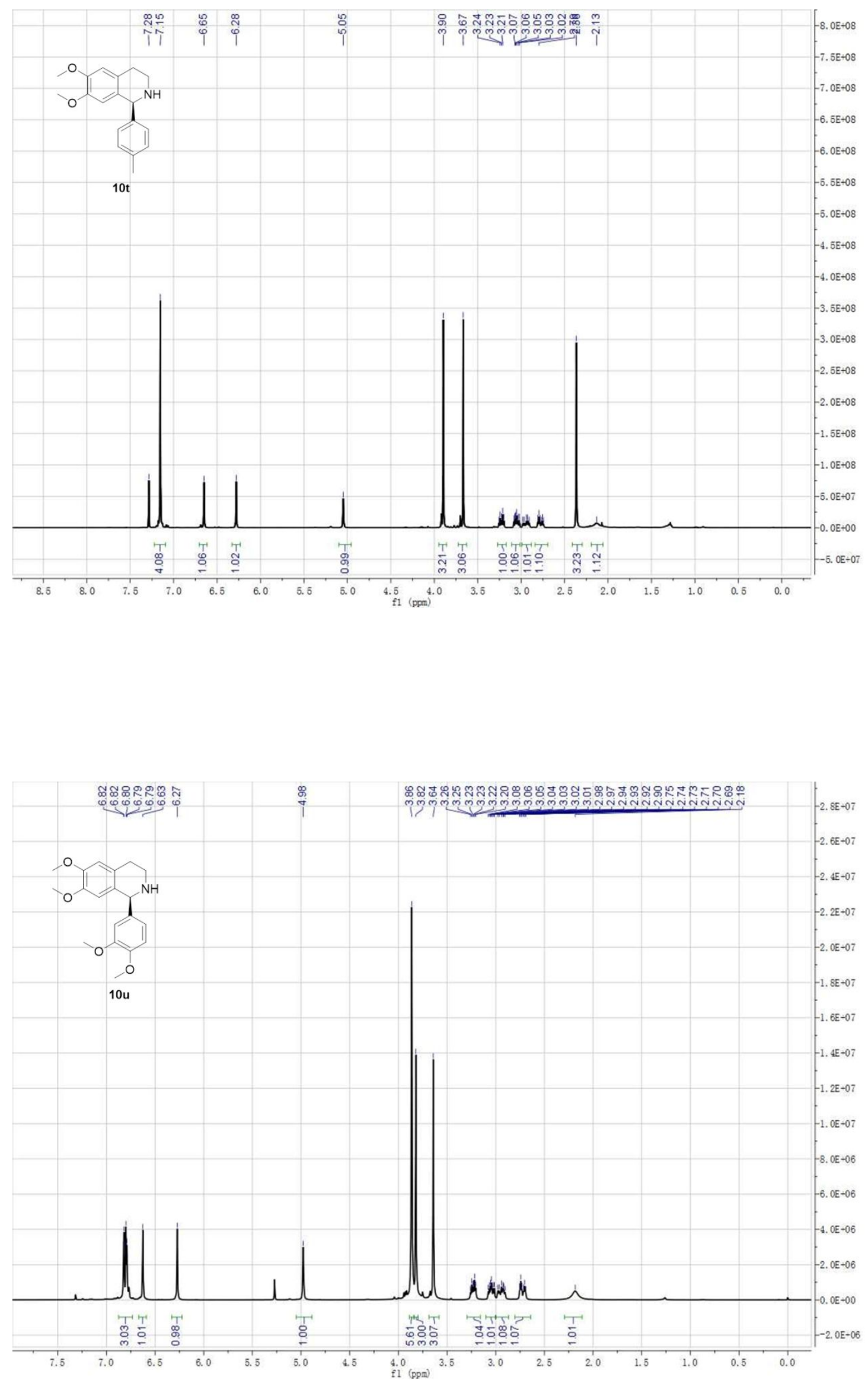

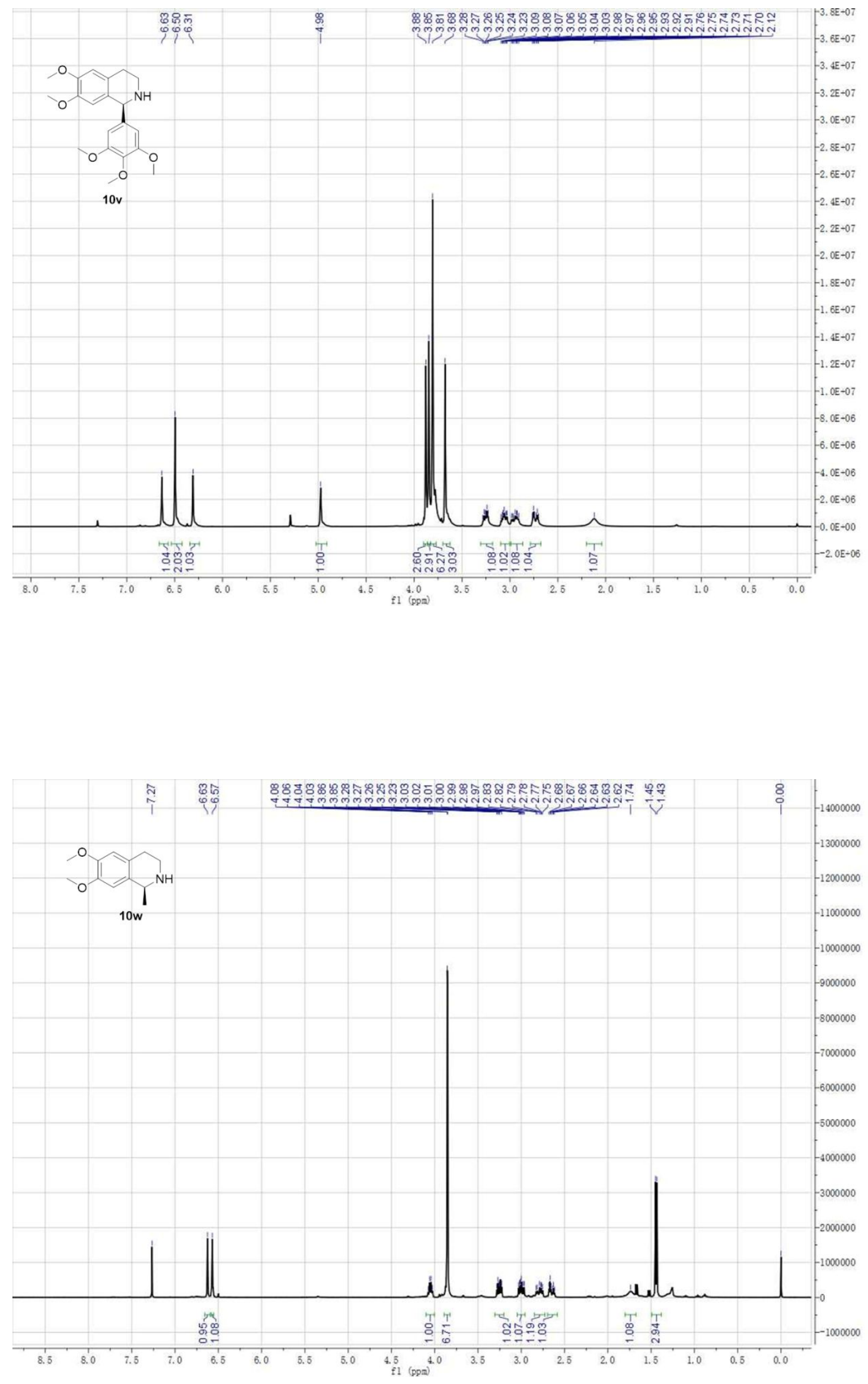


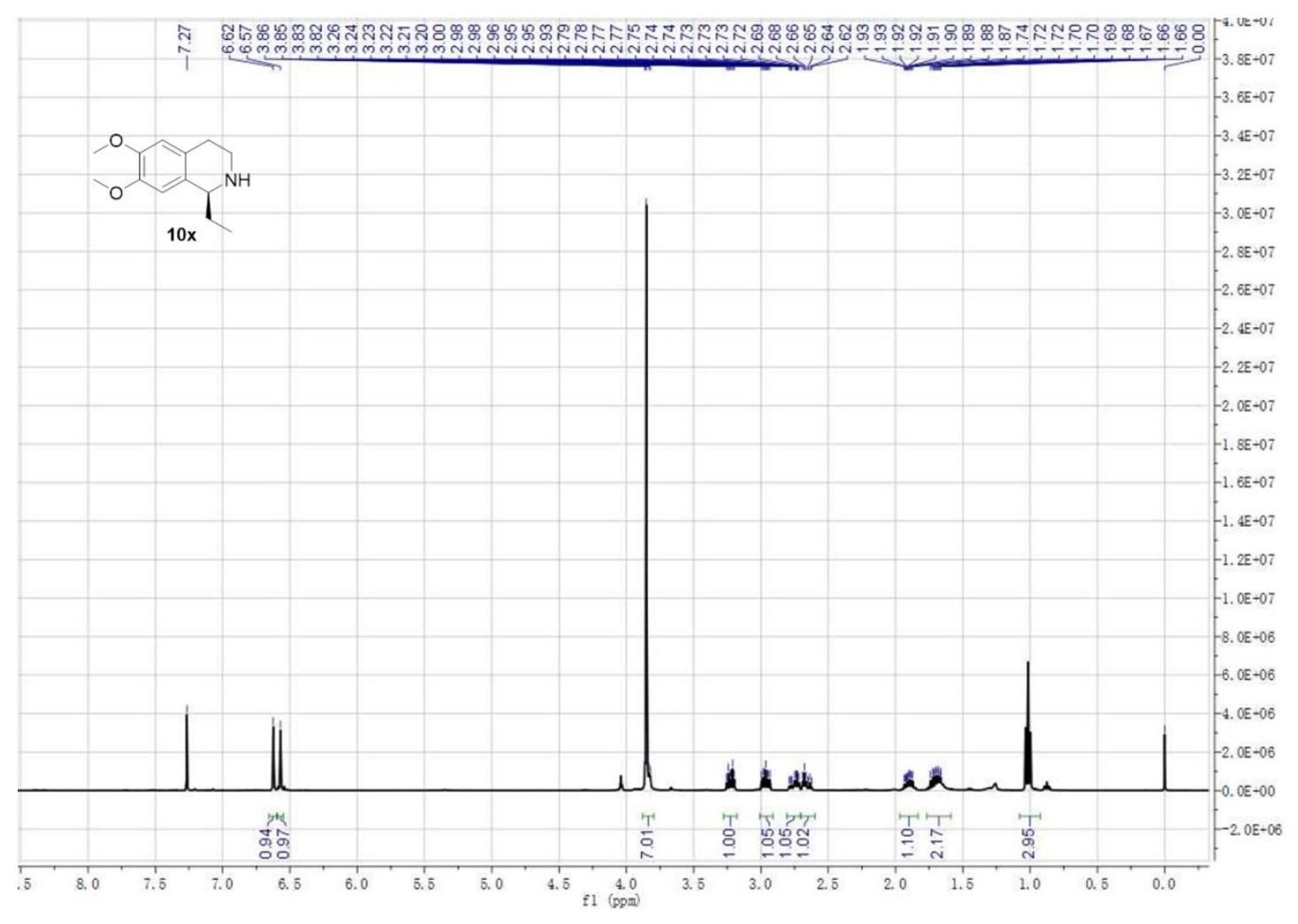




\section{HPLC spectra}
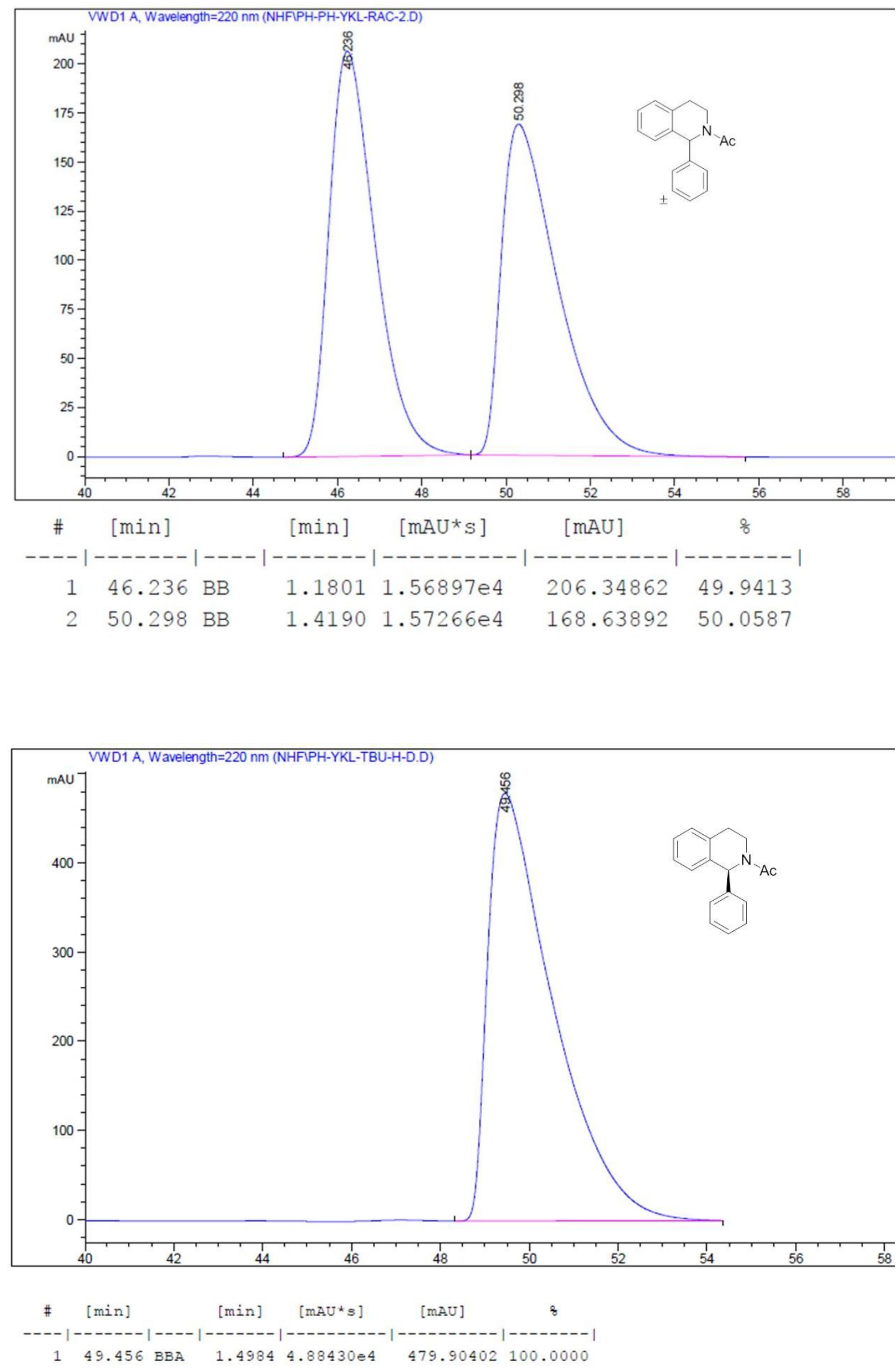

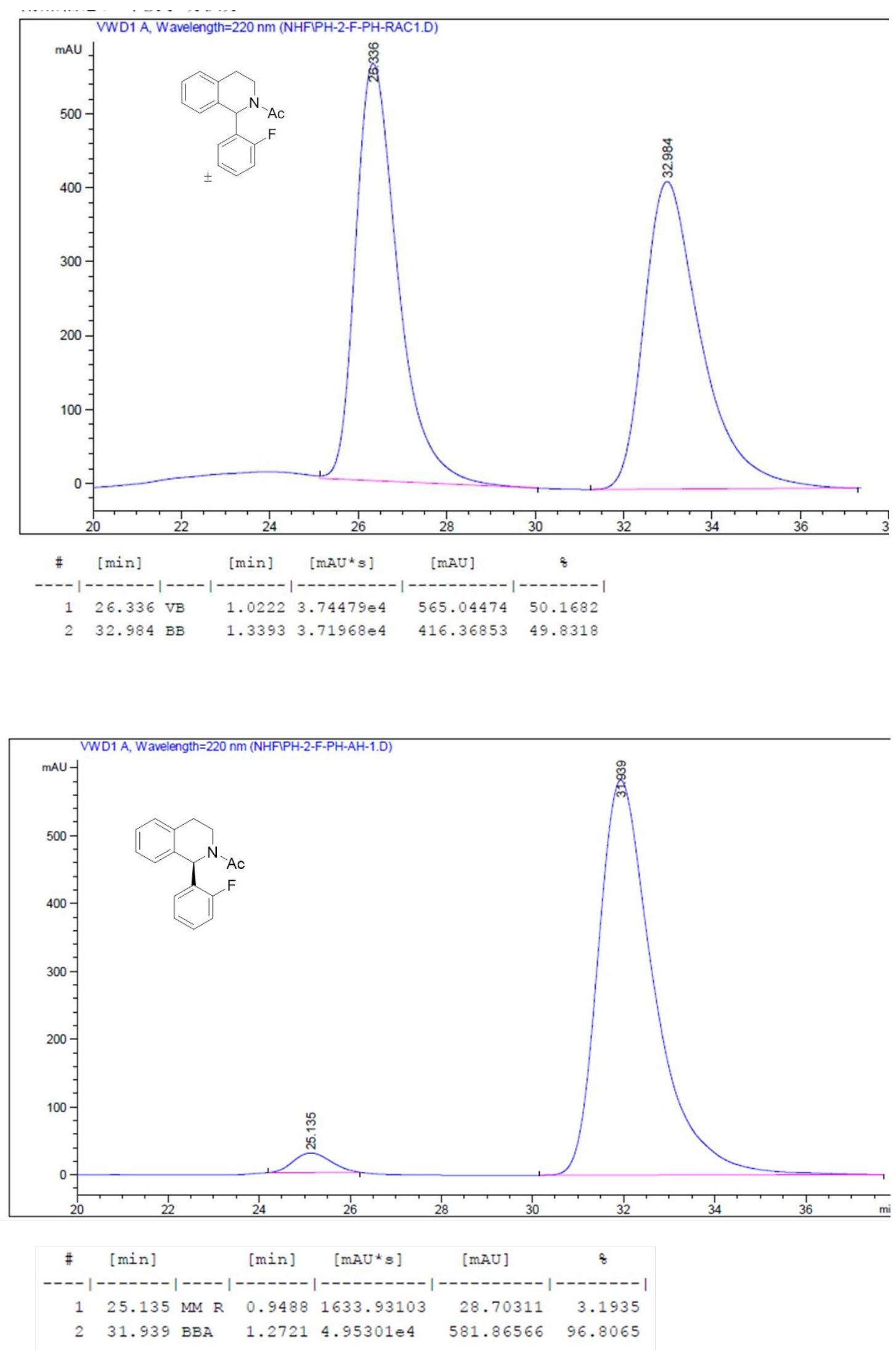

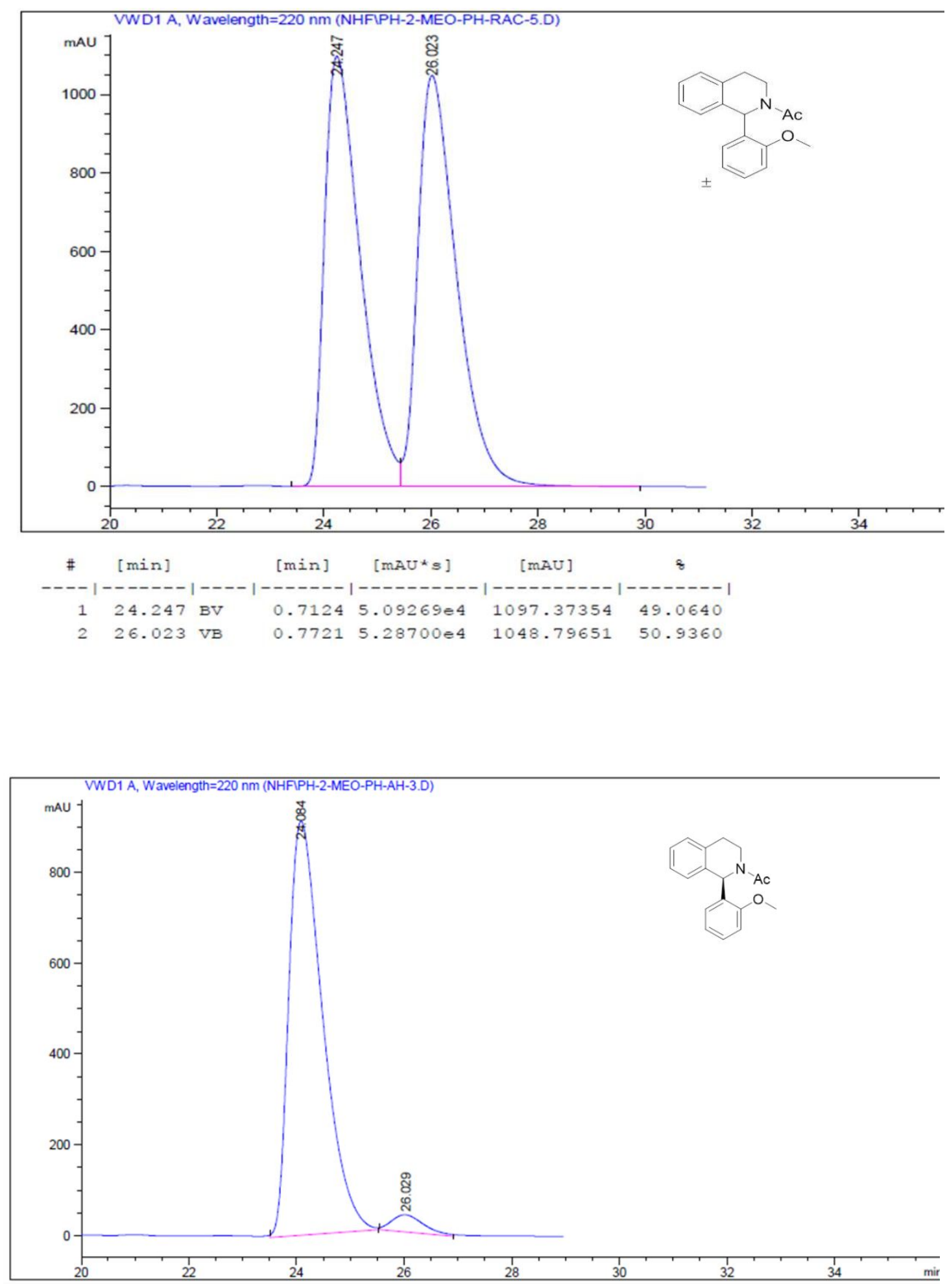
\# [min]
$[\mathrm{min}] \quad[\mathrm{mAU} * \mathrm{~s}]$
[MAU]
8
[
$\begin{array}{lllllll}1 & 24.084 & \text { MM R } & 0.7272 & 3.98355 e 4 & 913.01239 & 96.1868\end{array}$
$\begin{array}{lllllll}2 & 26.029 & \text { MM R } & 0.6969 & 1579.24353 & 37.77035 & 3.8132\end{array}$ 

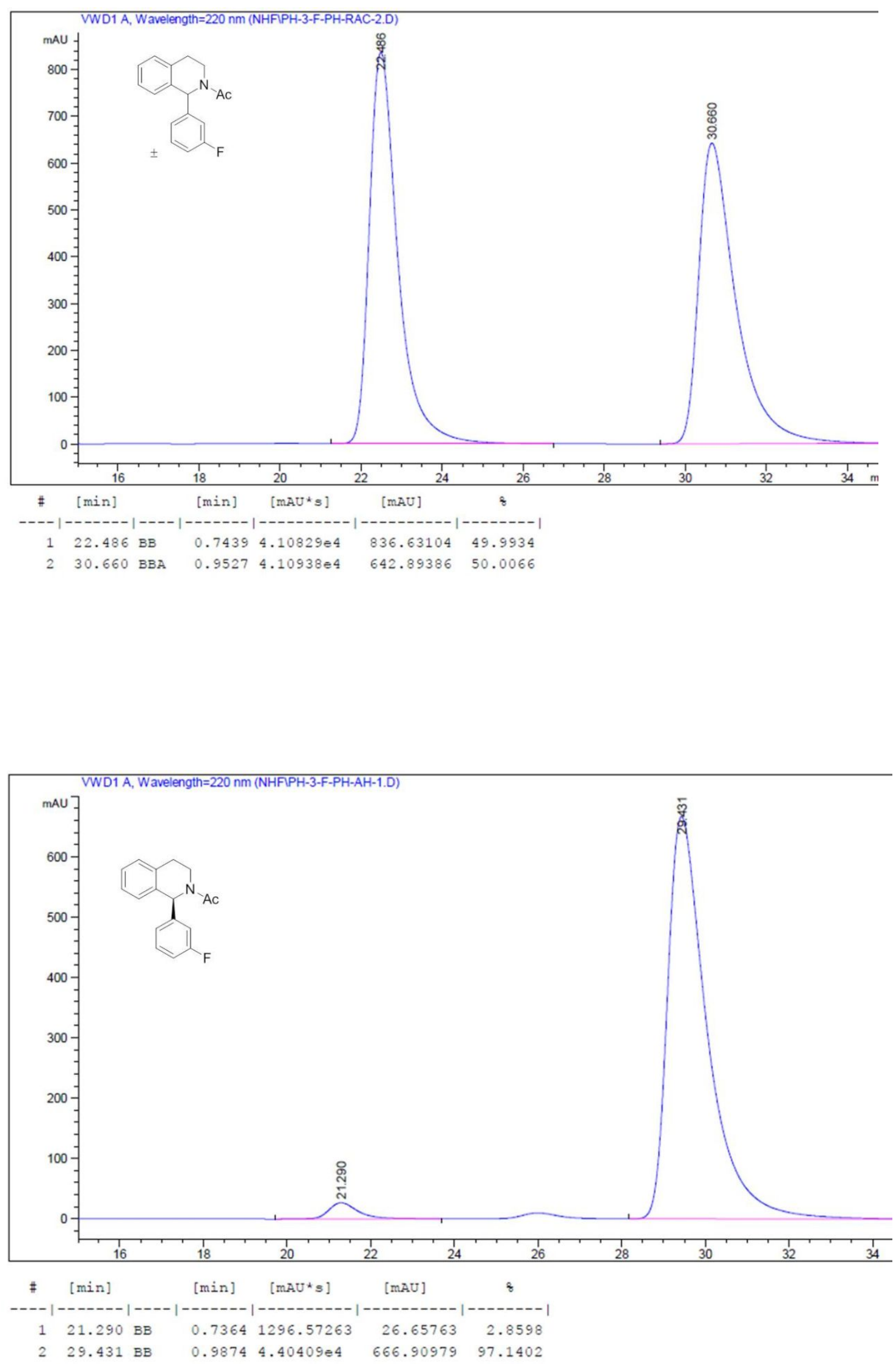

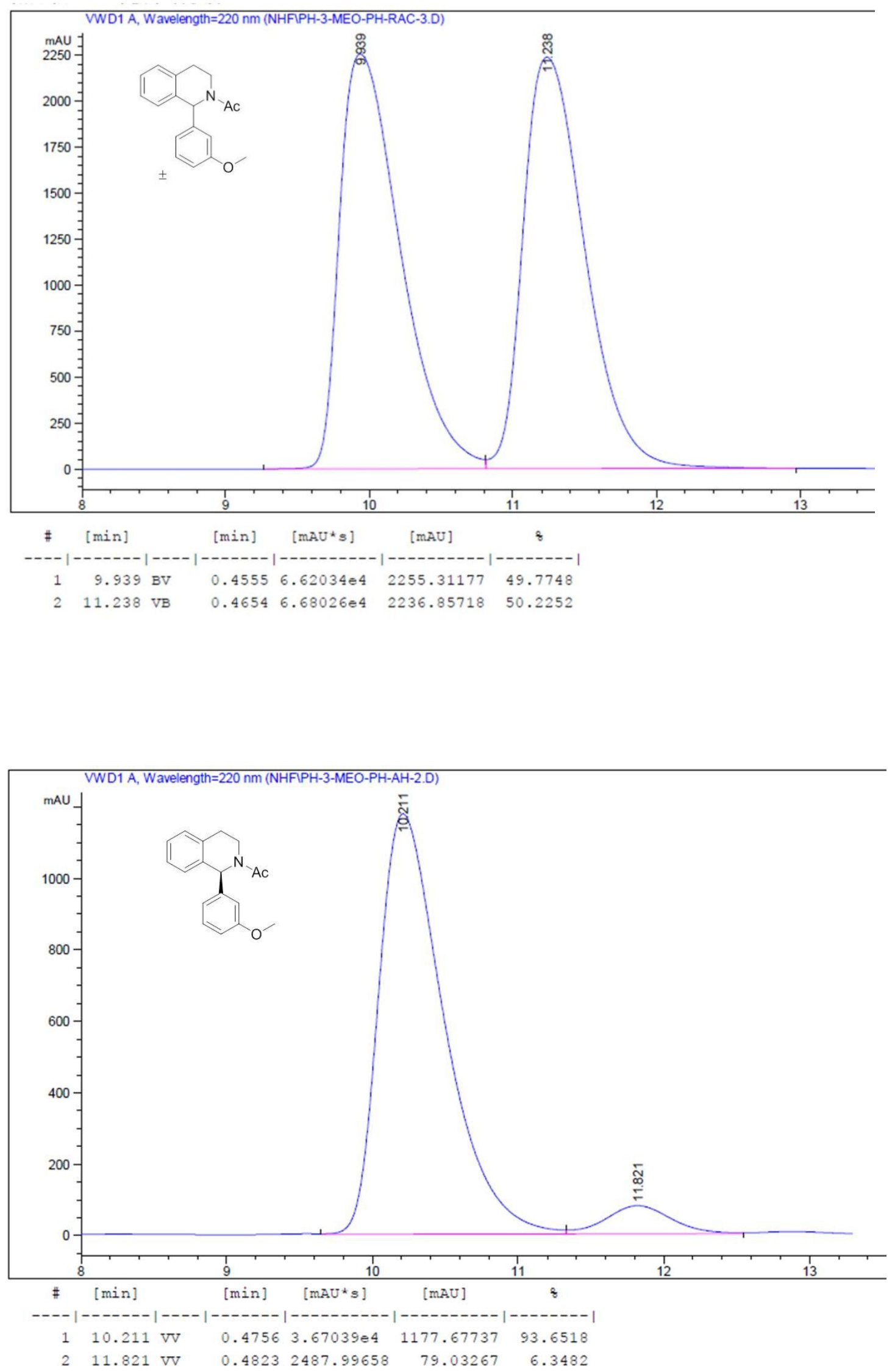

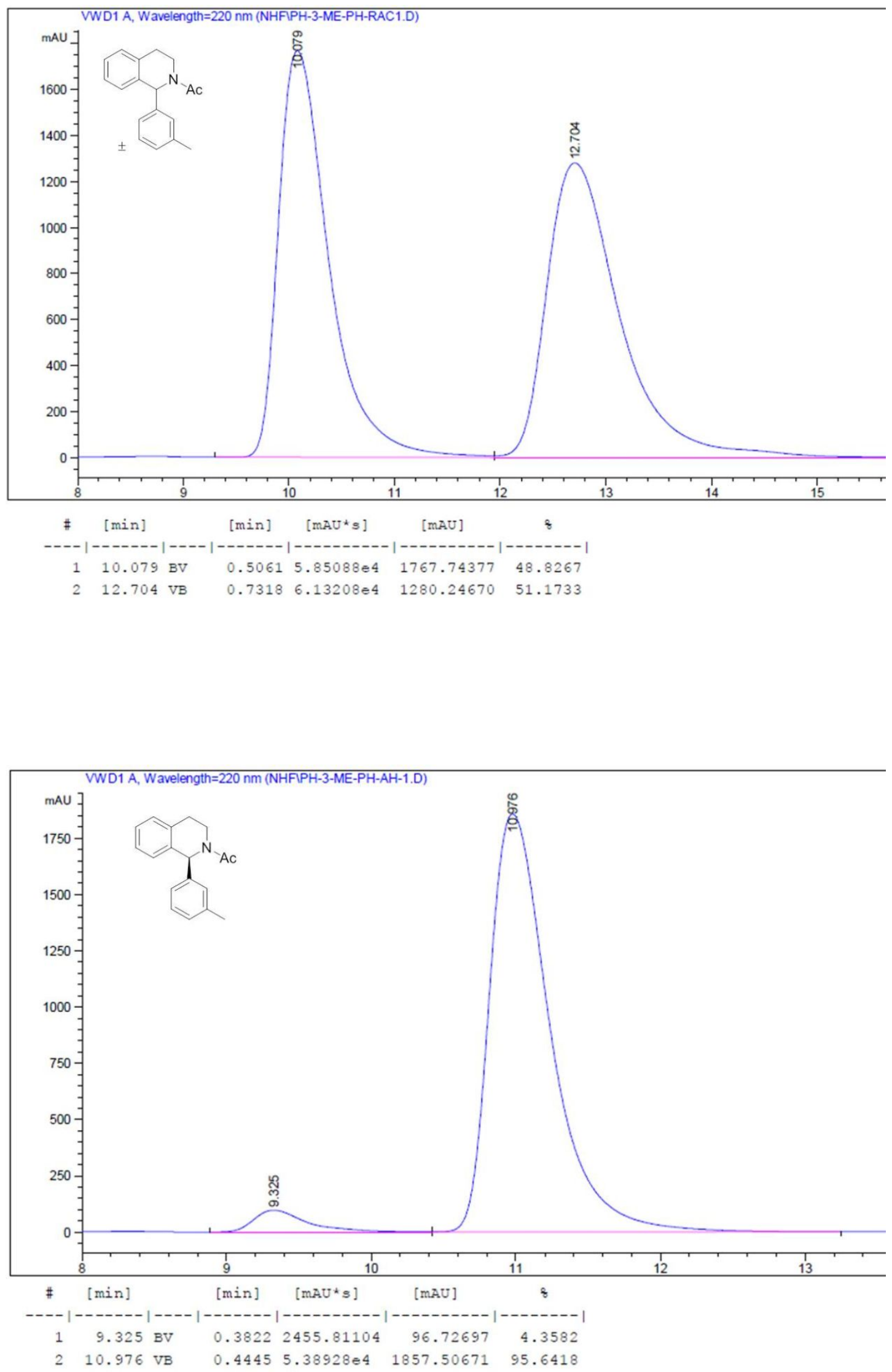

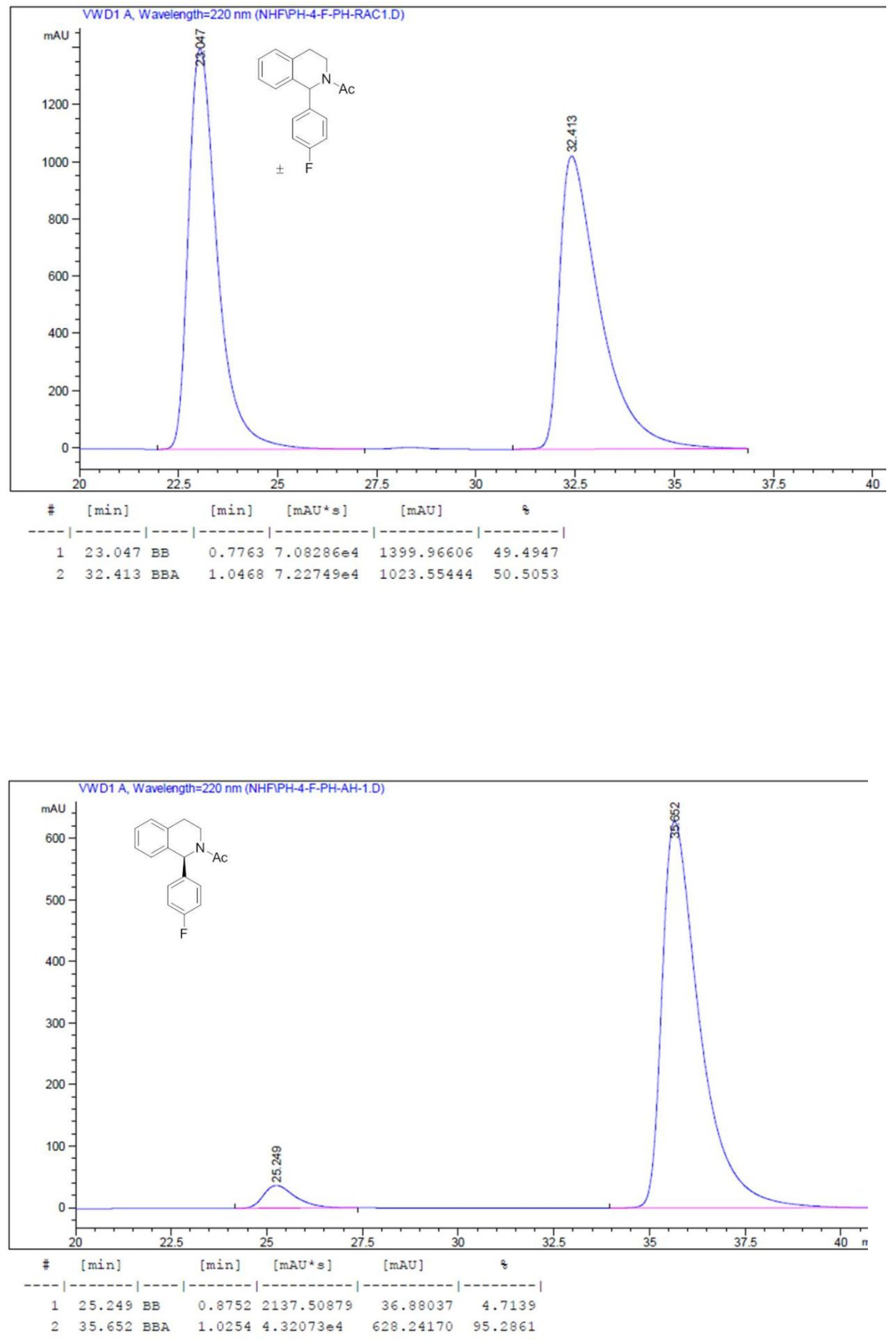


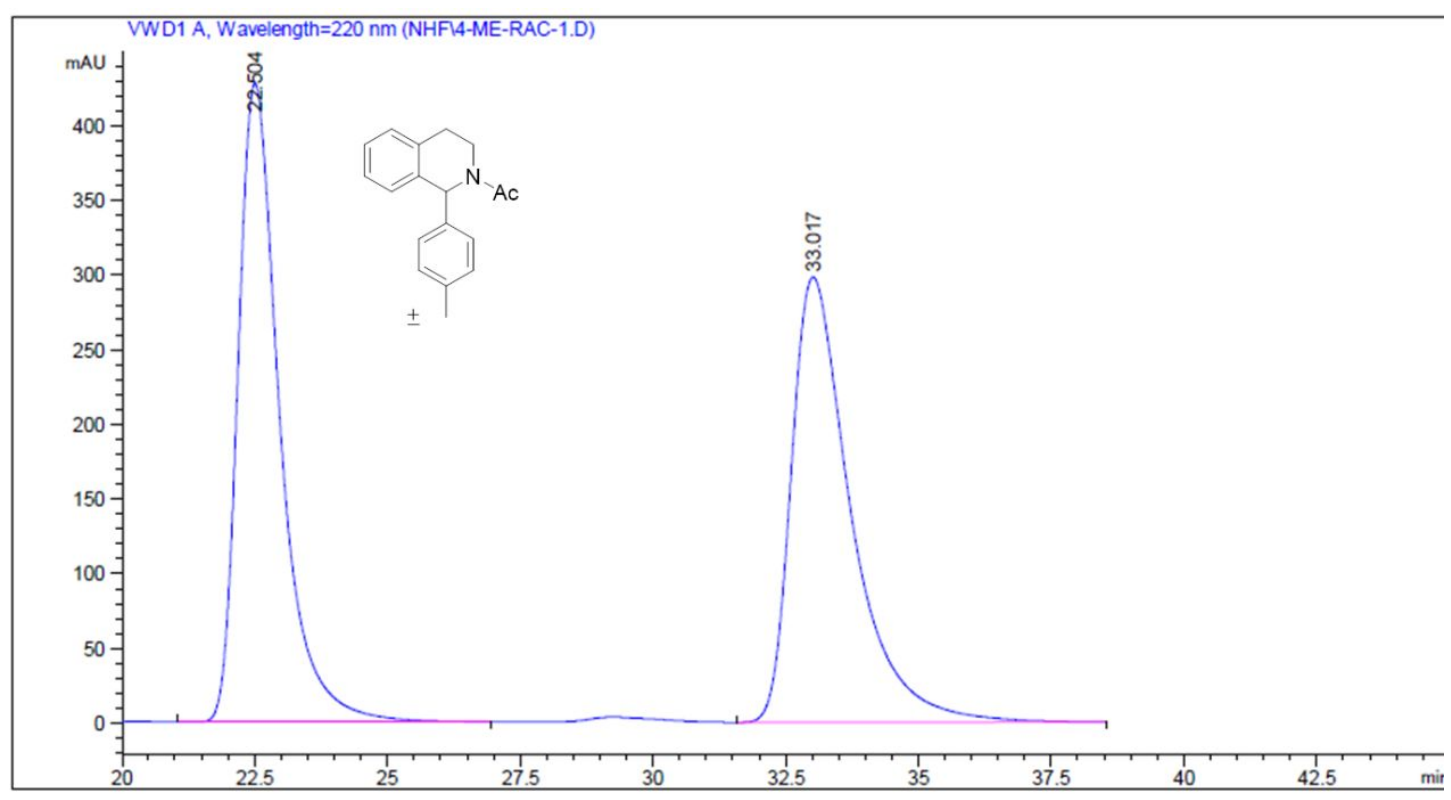

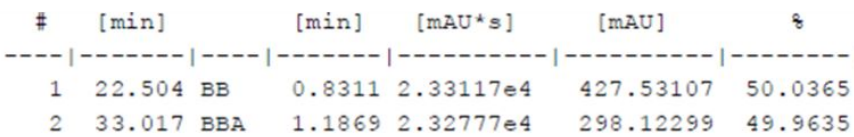

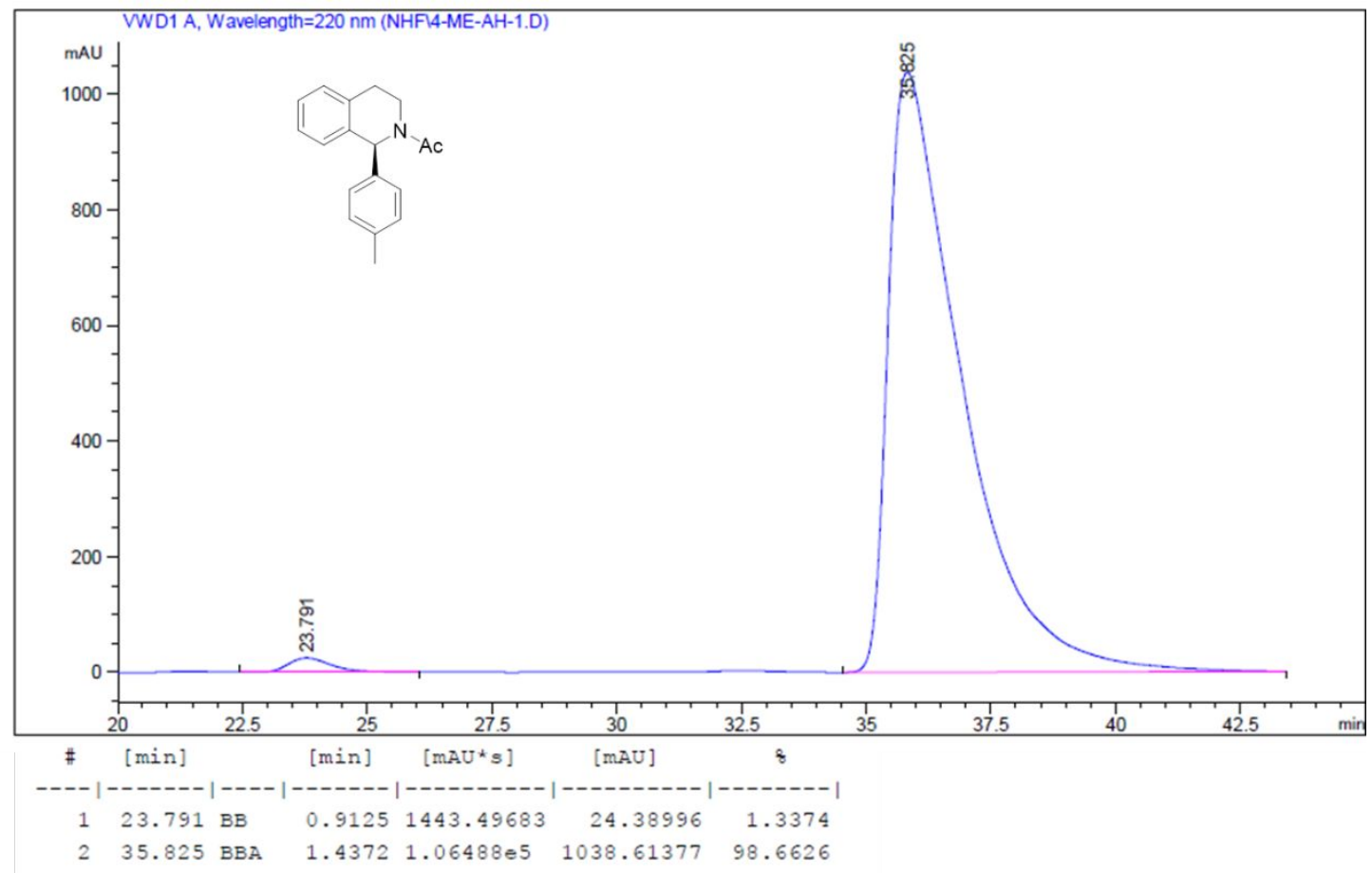



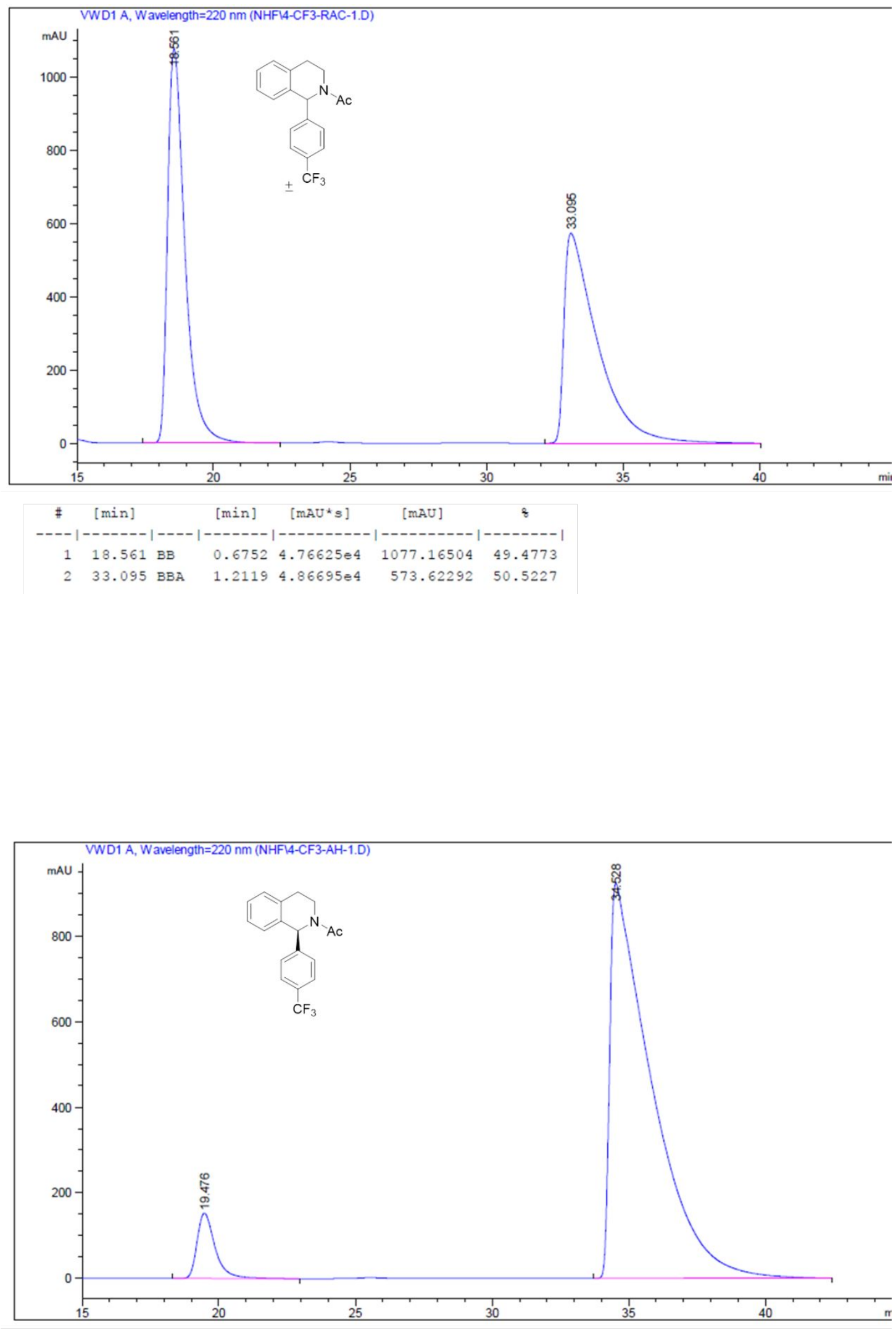

\# $[\mathrm{min}] \quad[\mathrm{min}] \quad[\mathrm{mAU} * \mathrm{~s}] \quad[\mathrm{mAU}]$ ----|-------|----|-------|----------|----------1--------

$\begin{array}{lllllll}1 & 19.476 & \mathrm{BB} & 0.7020 & 6988.89258 & 152.40936 & 6.6367\end{array}$

$2 \quad 34.528$ BBA $\quad 1.3645 \quad 9.83173 e 4 \quad 923.14661 \quad 93.3633$ 

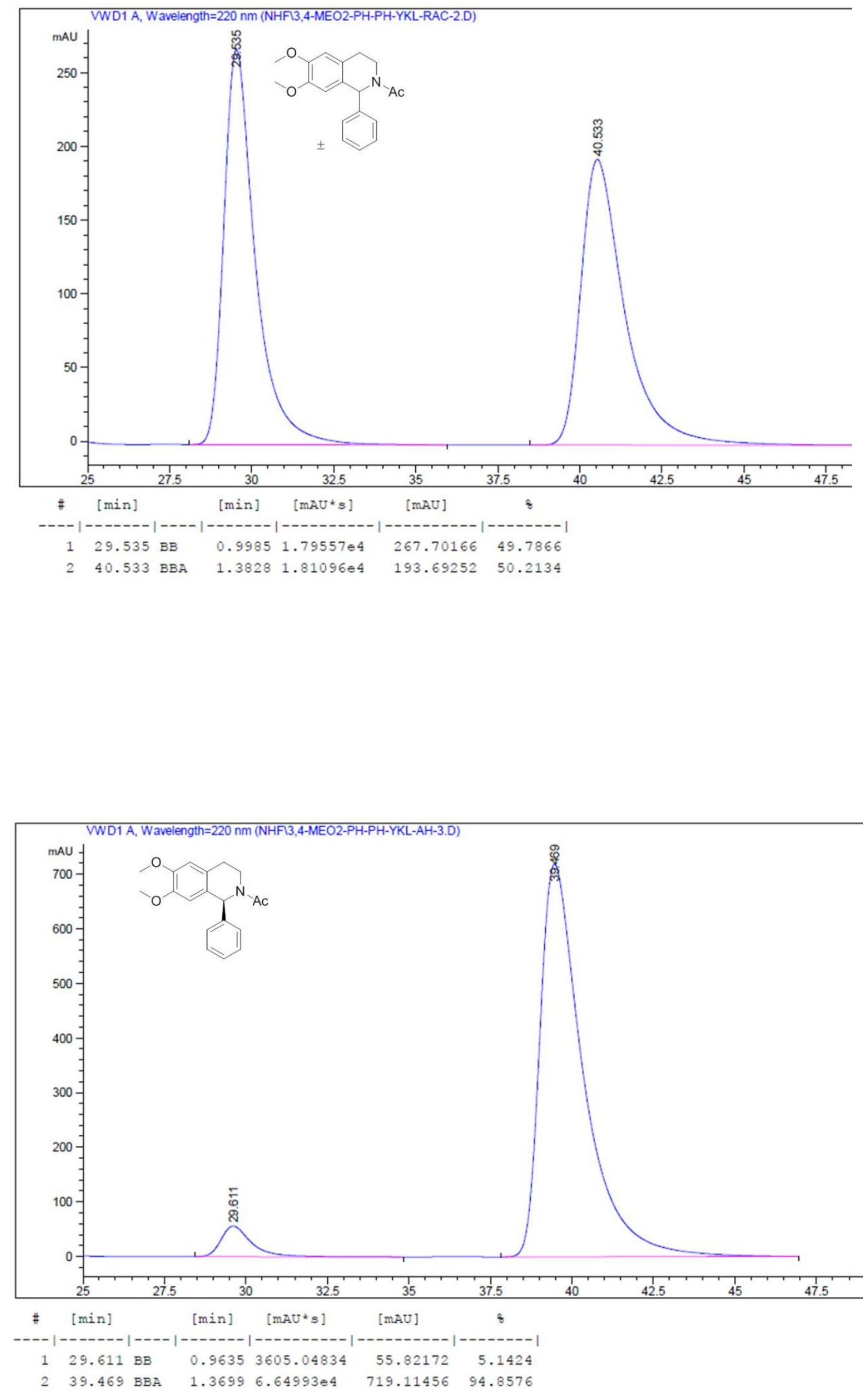

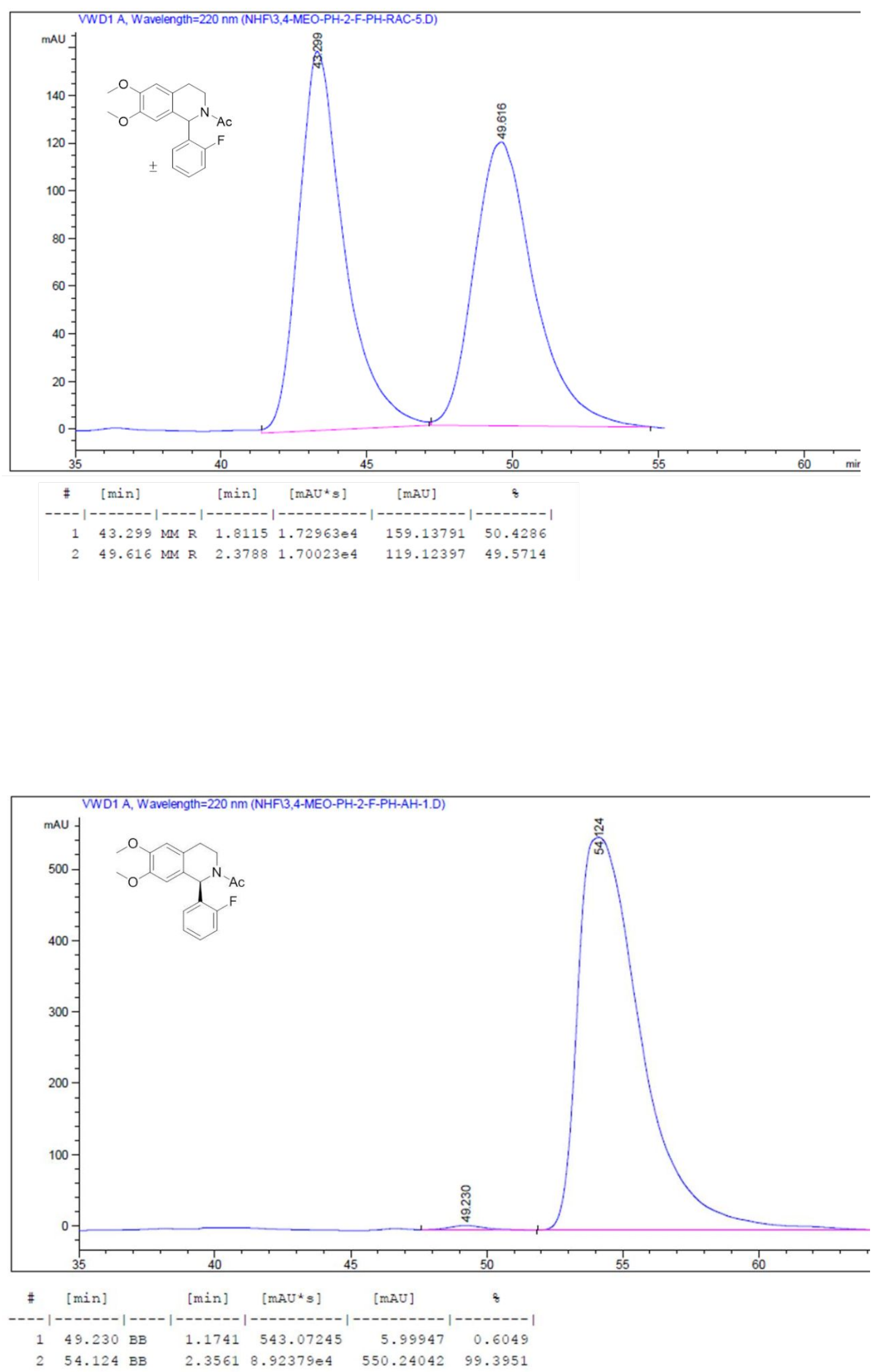

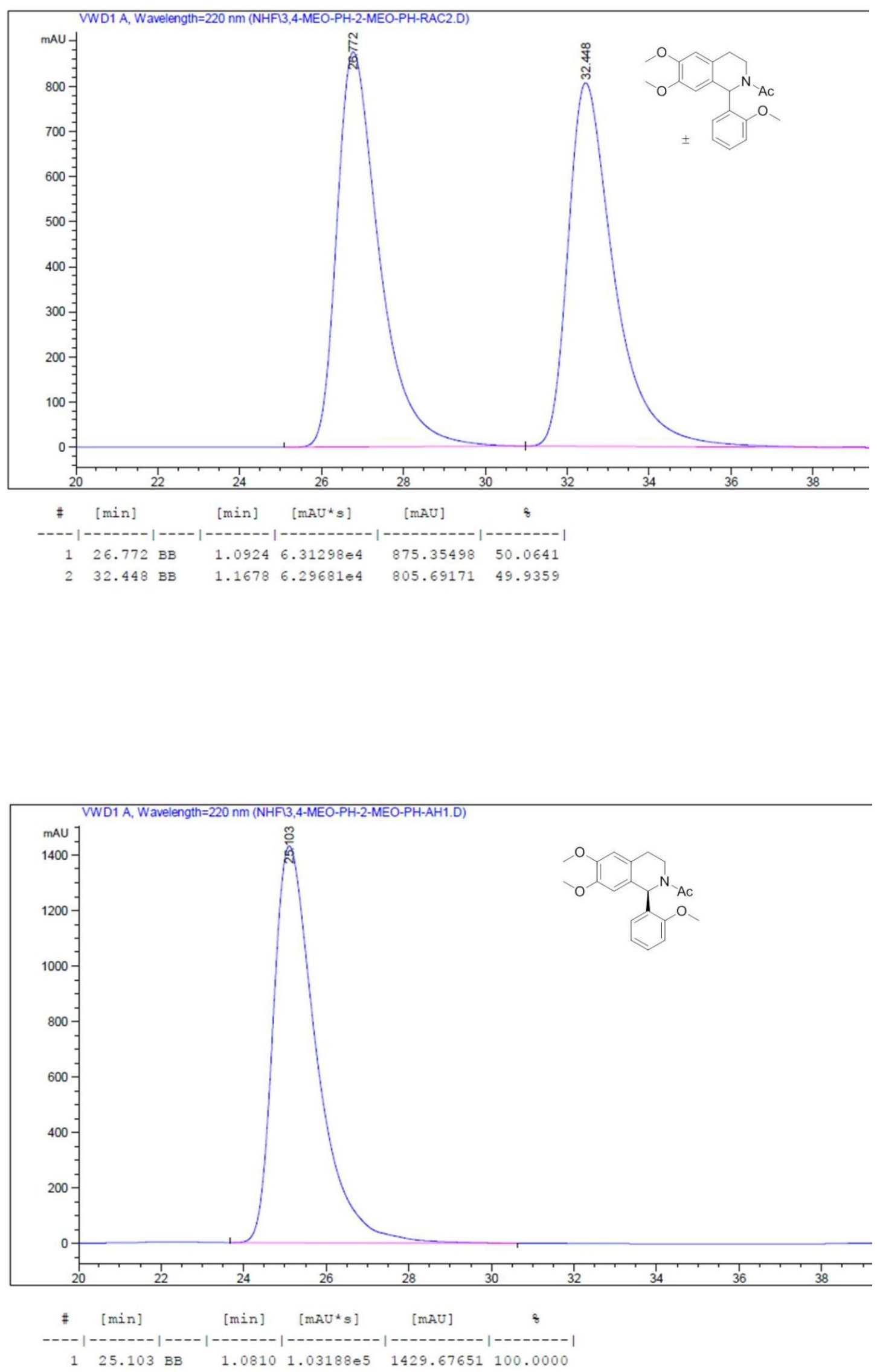

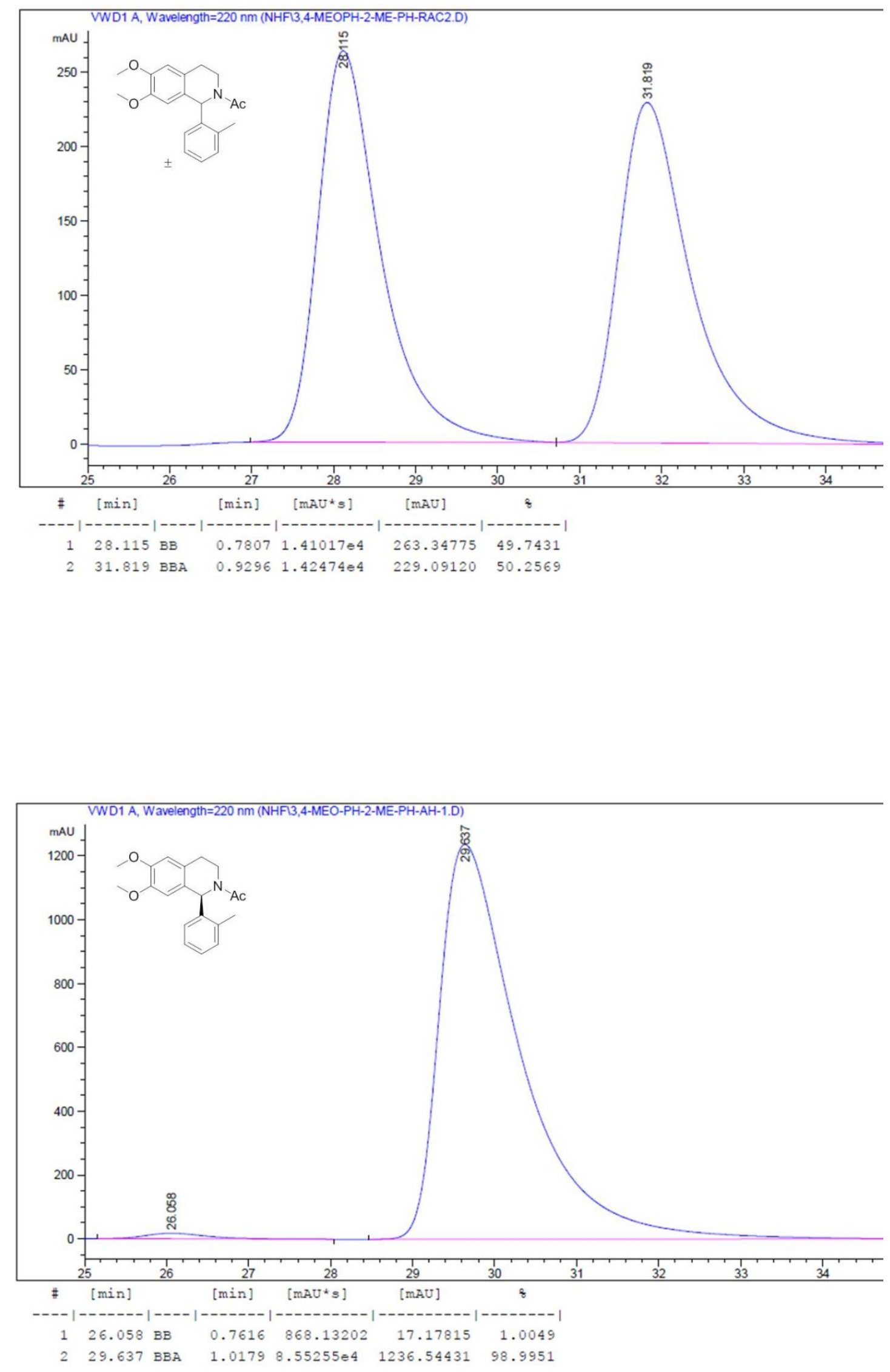

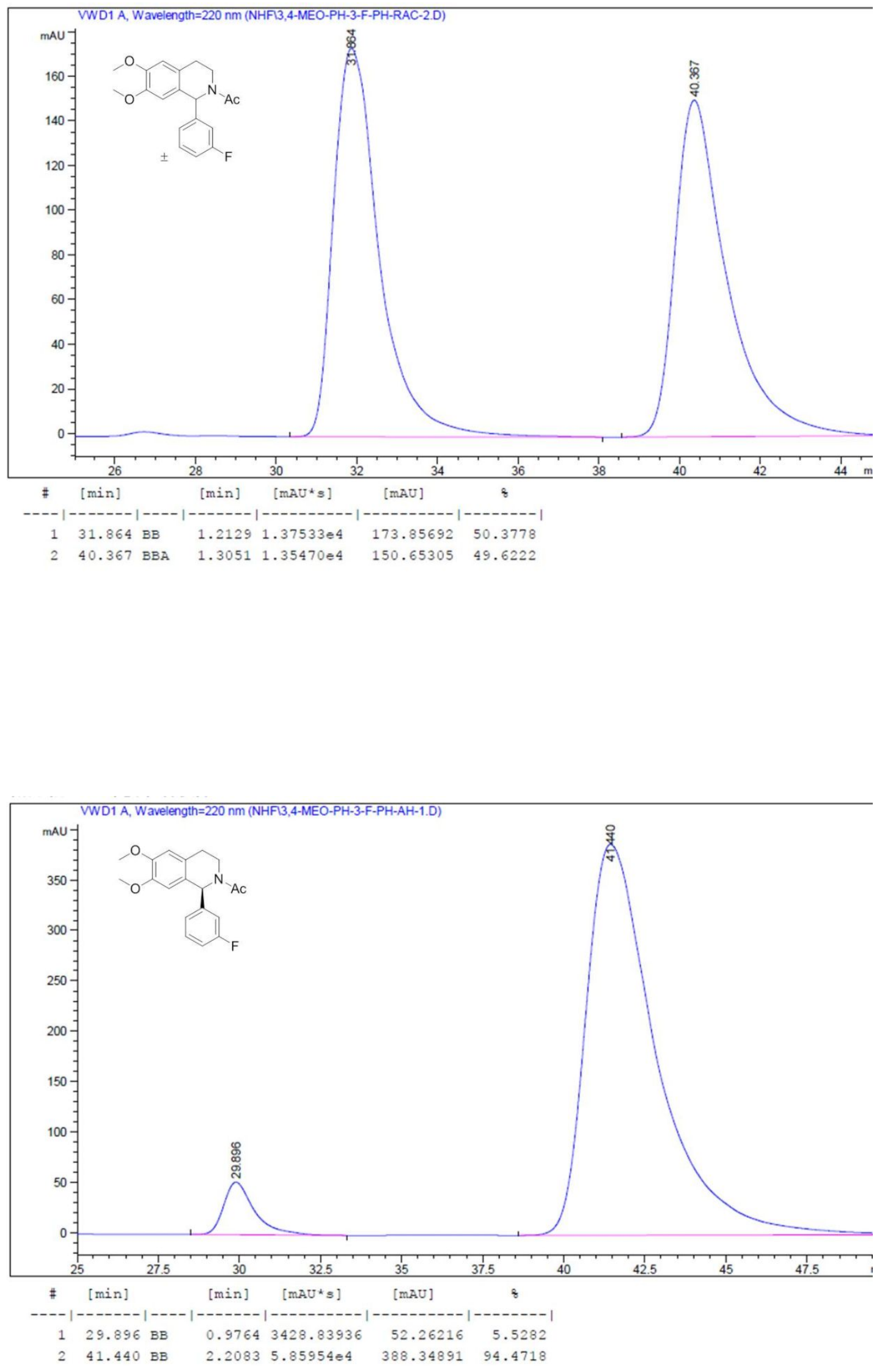

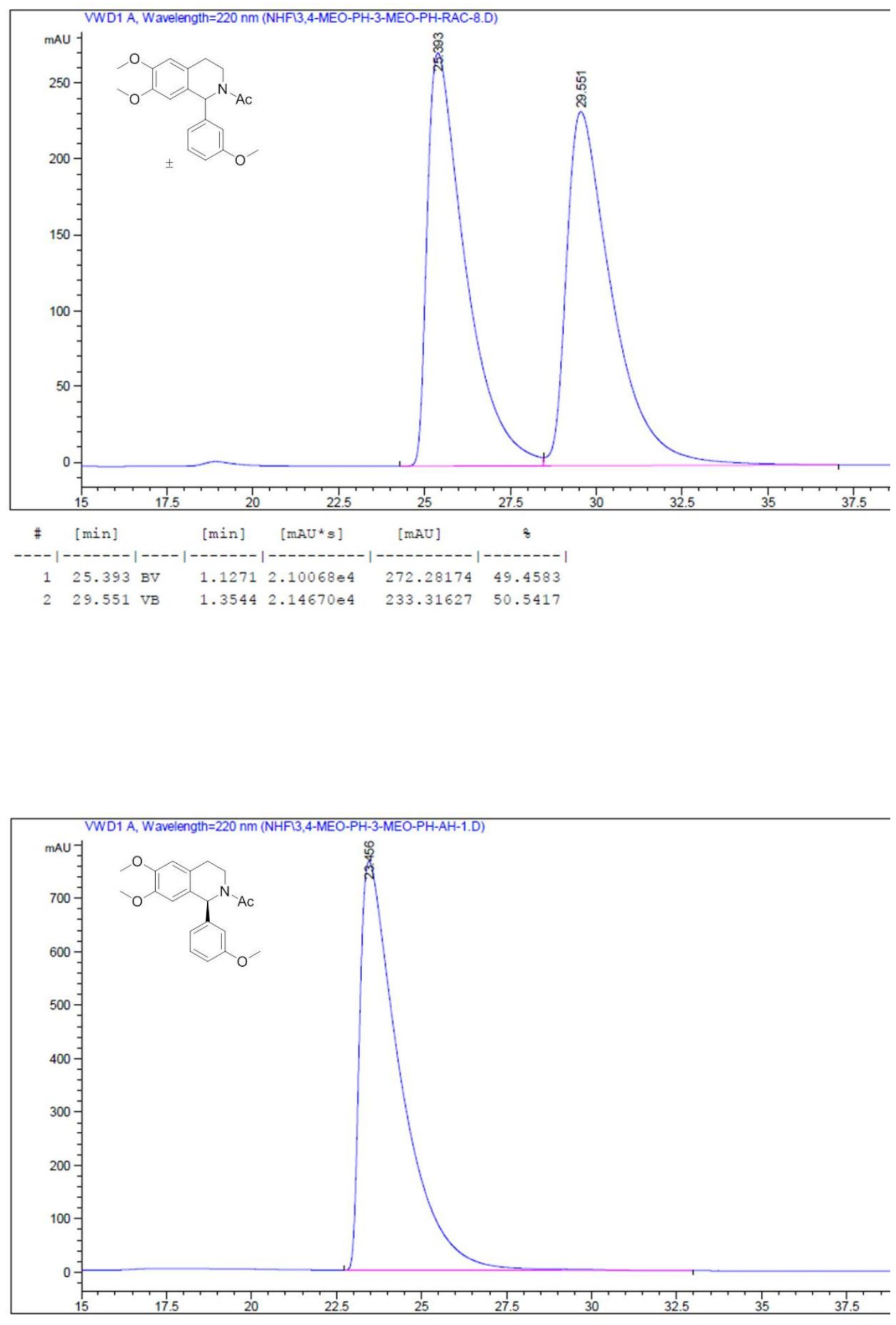

\# $[\mathrm{min}] \quad[\mathrm{min}] \quad[\mathrm{mAU} * \mathrm{~s}] \quad[\mathrm{mAU}]$

----|--------|-----|--------|------------|-----------|---------|

$\begin{array}{llllll}1 & 23.456 \mathrm{BB} & 1.1931 & 6.30659 e 4 & 765.53101 & 100.0000\end{array}$ 

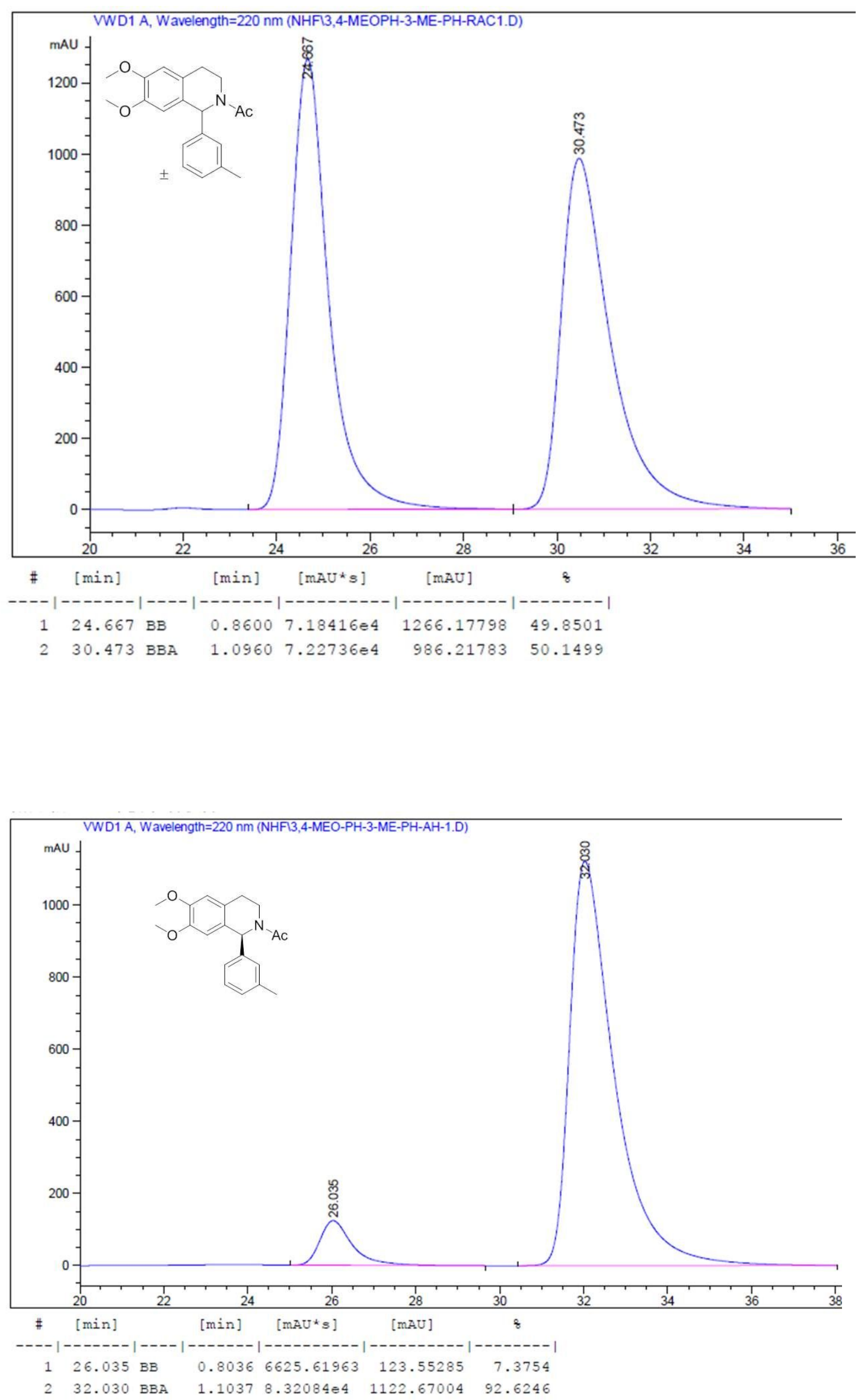

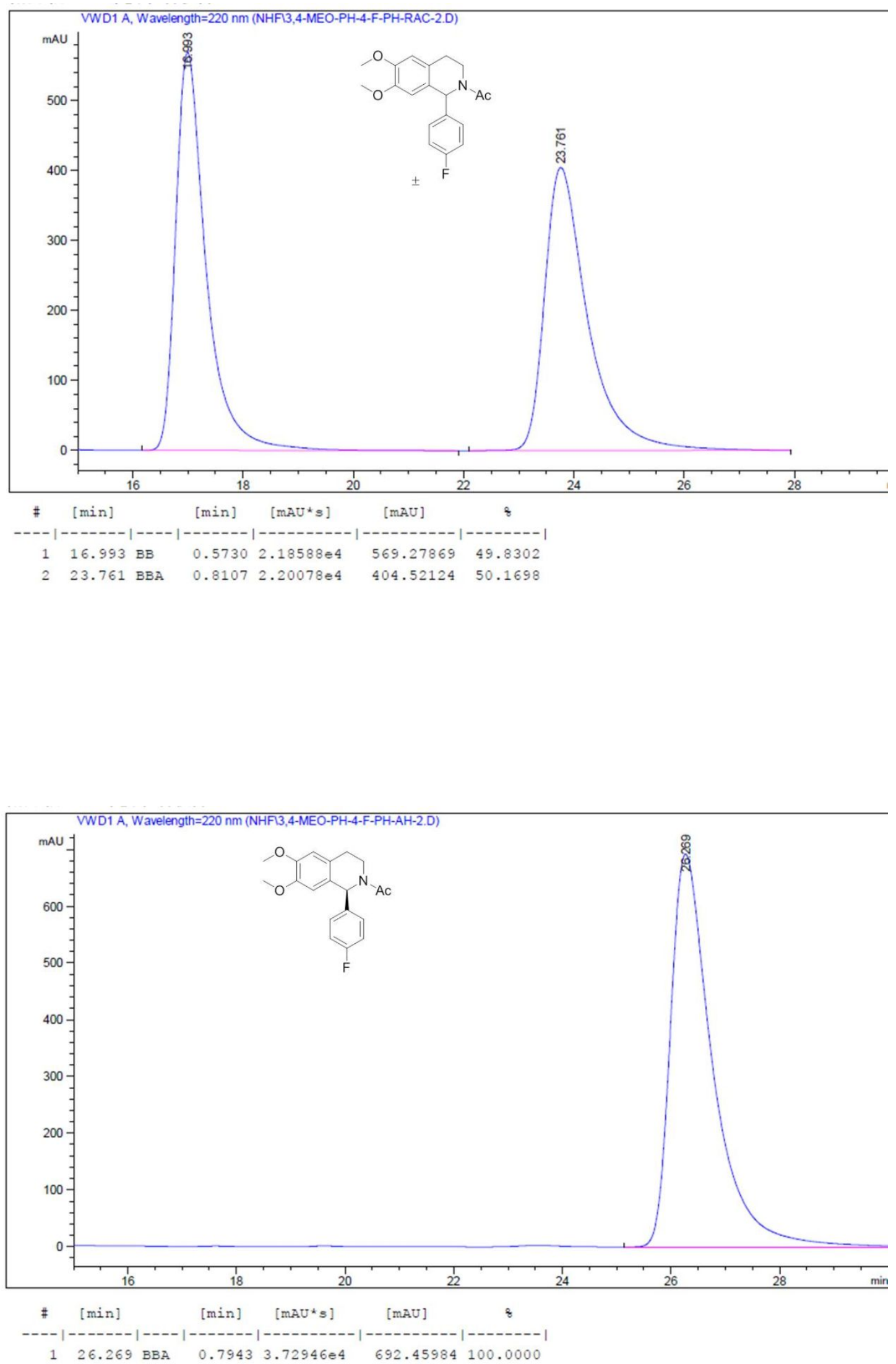

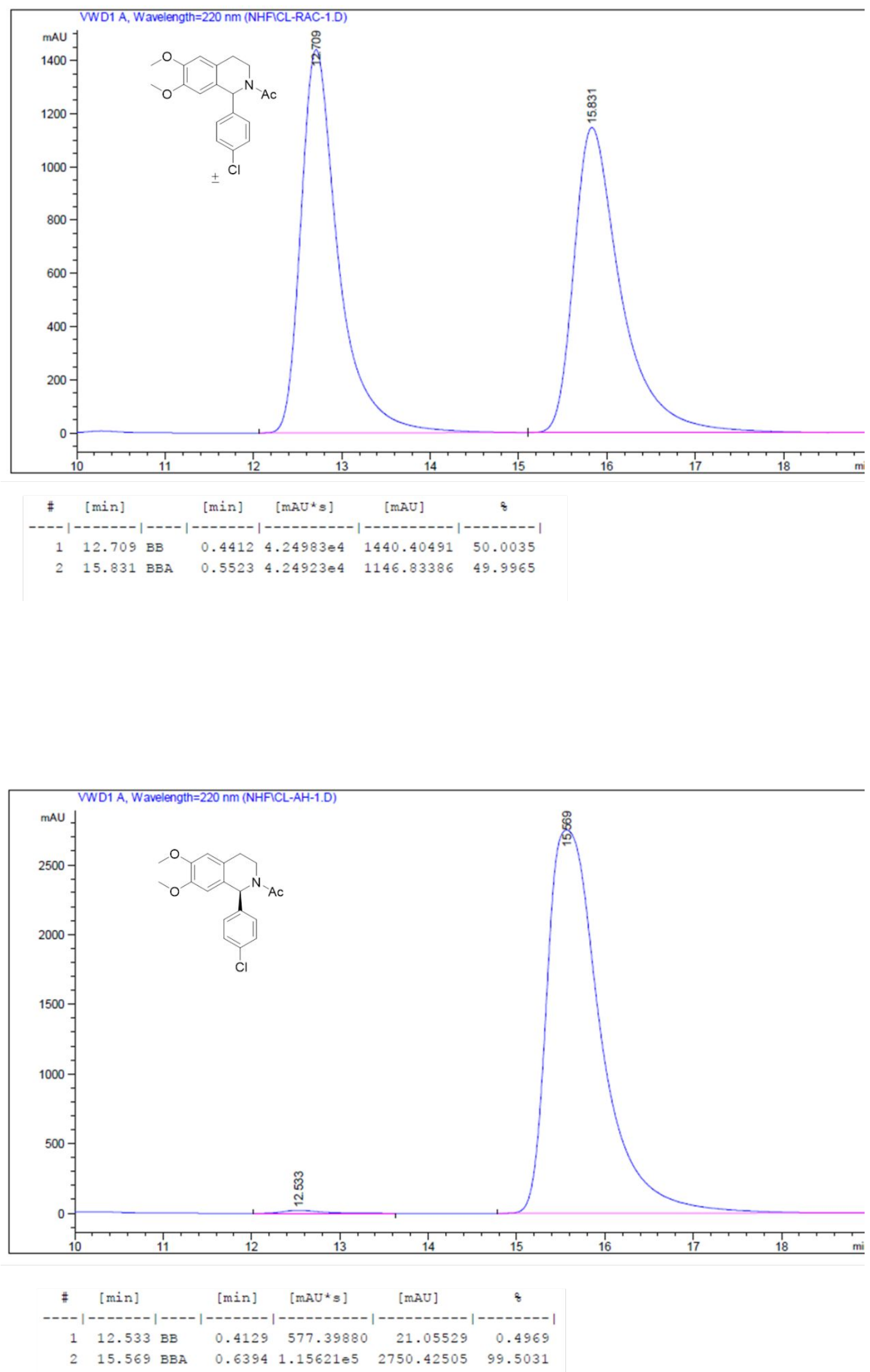

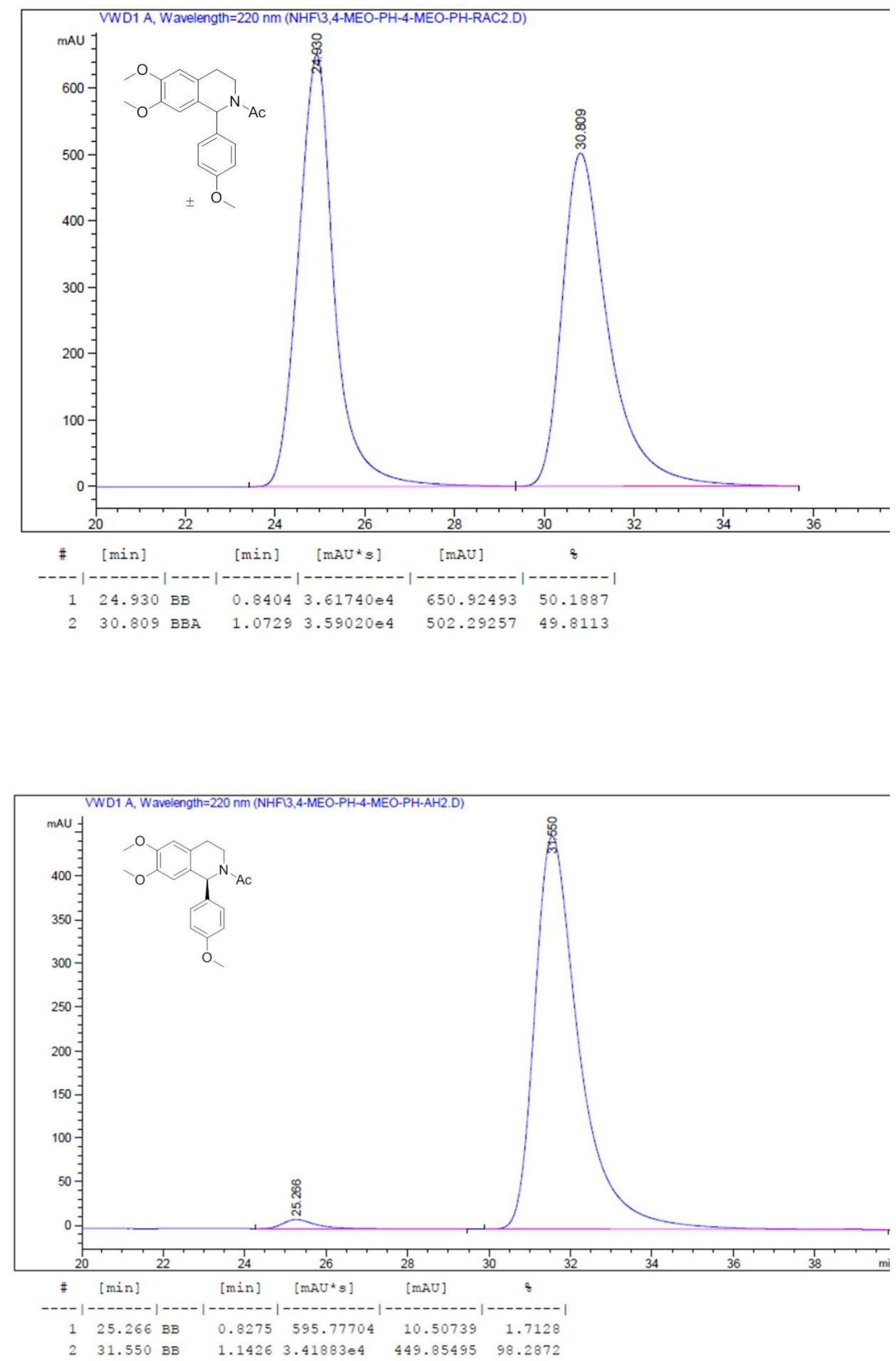

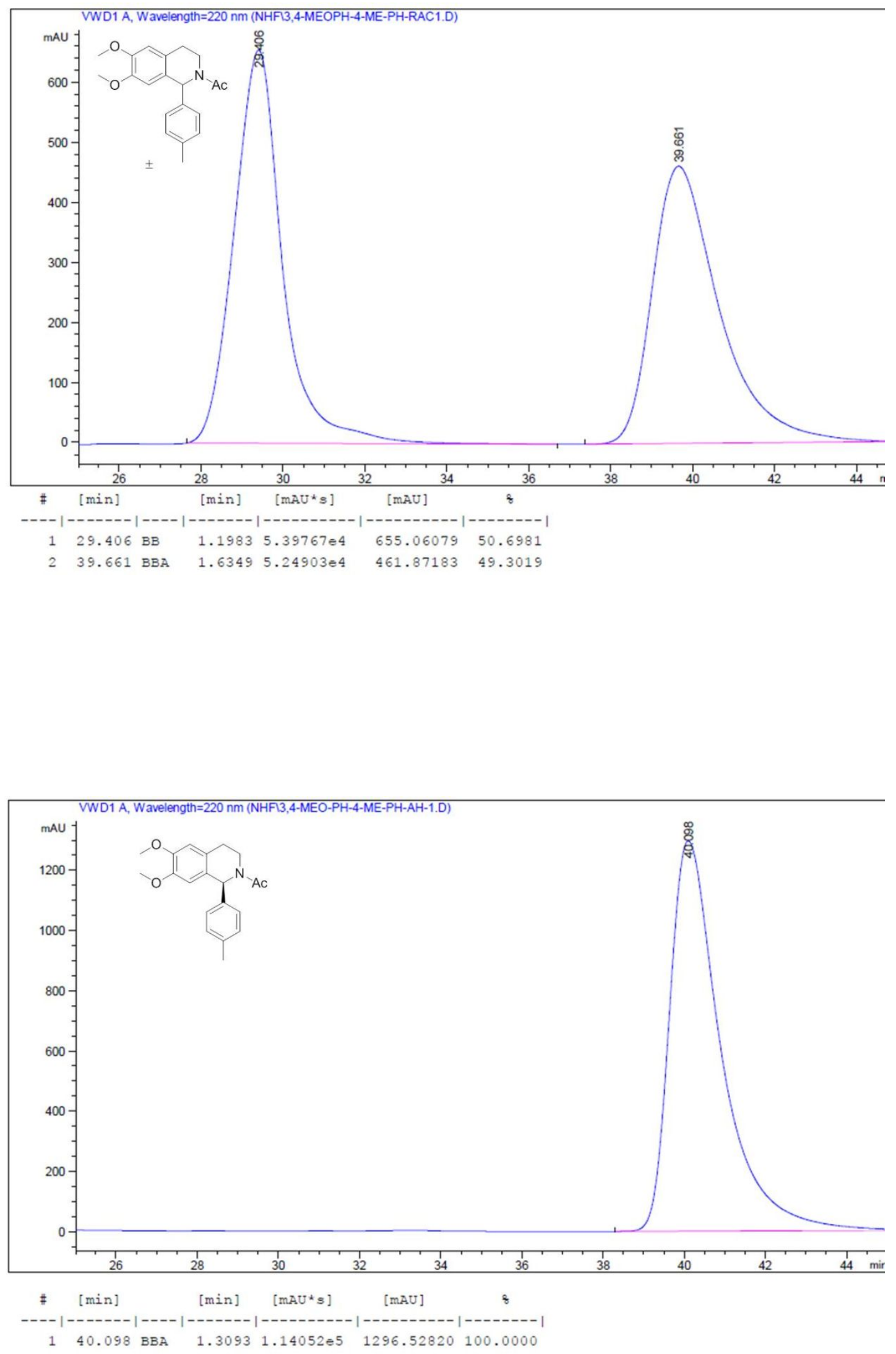

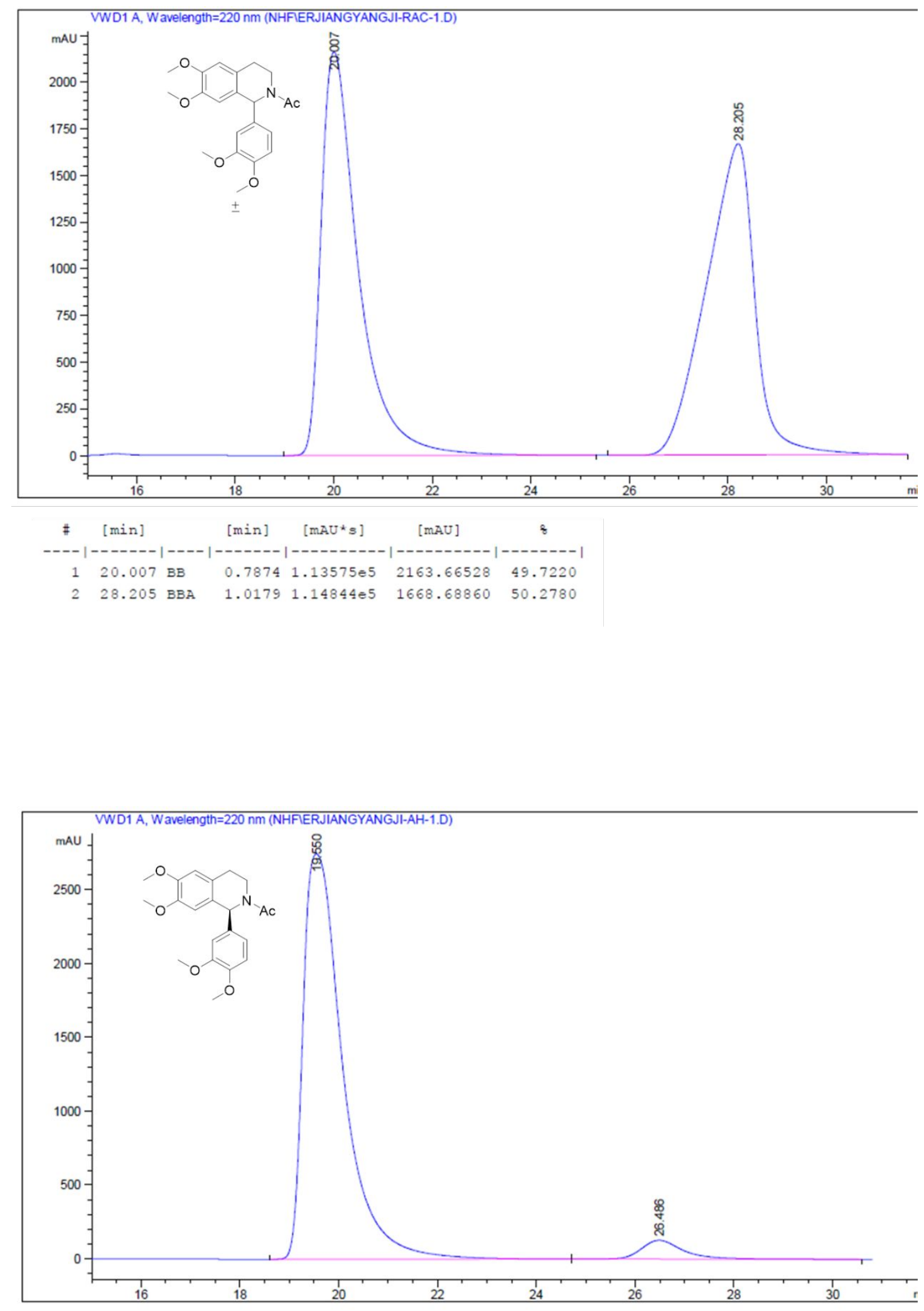

\begin{tabular}{|c|c|c|c|c|c|}
\hline \# & [min] & [min] & {$[\mathrm{mAU} * \mathrm{~s}]$} & [mAU] & 8 \\
\hline-1 & $-------\mid--$ & ------- & 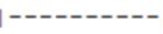 & 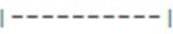 & $--------\mid$ \\
\hline 1 & $19.550 \mathrm{BB}$ & 0.8467 & 1.53505 es & 2743.91479 & 95.2707 \\
\hline 2 & $26.486 \mathrm{BB}$ & 0.8909 & 7620.12305 & 127.75839 & 4.7293 \\
\hline
\end{tabular}



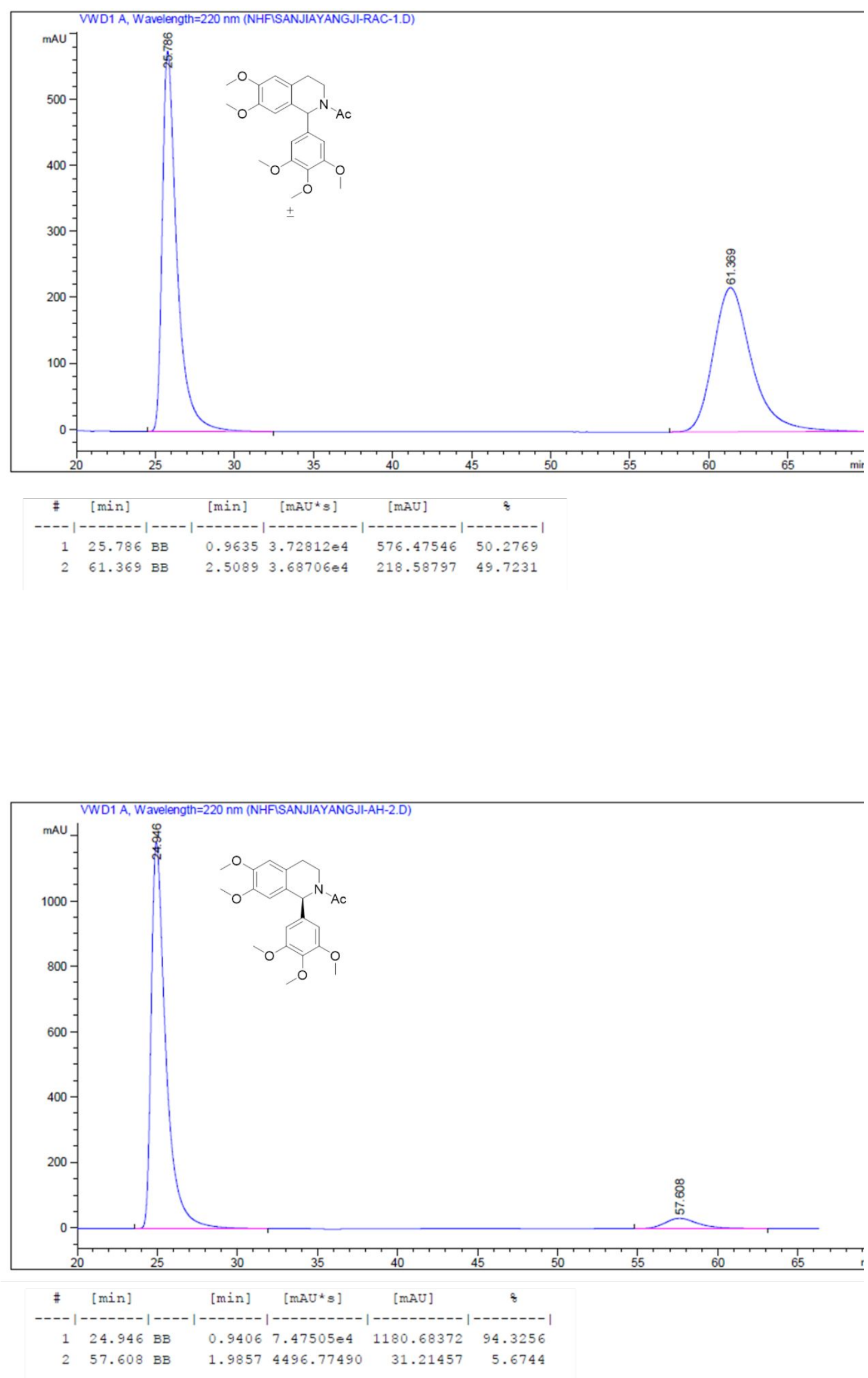

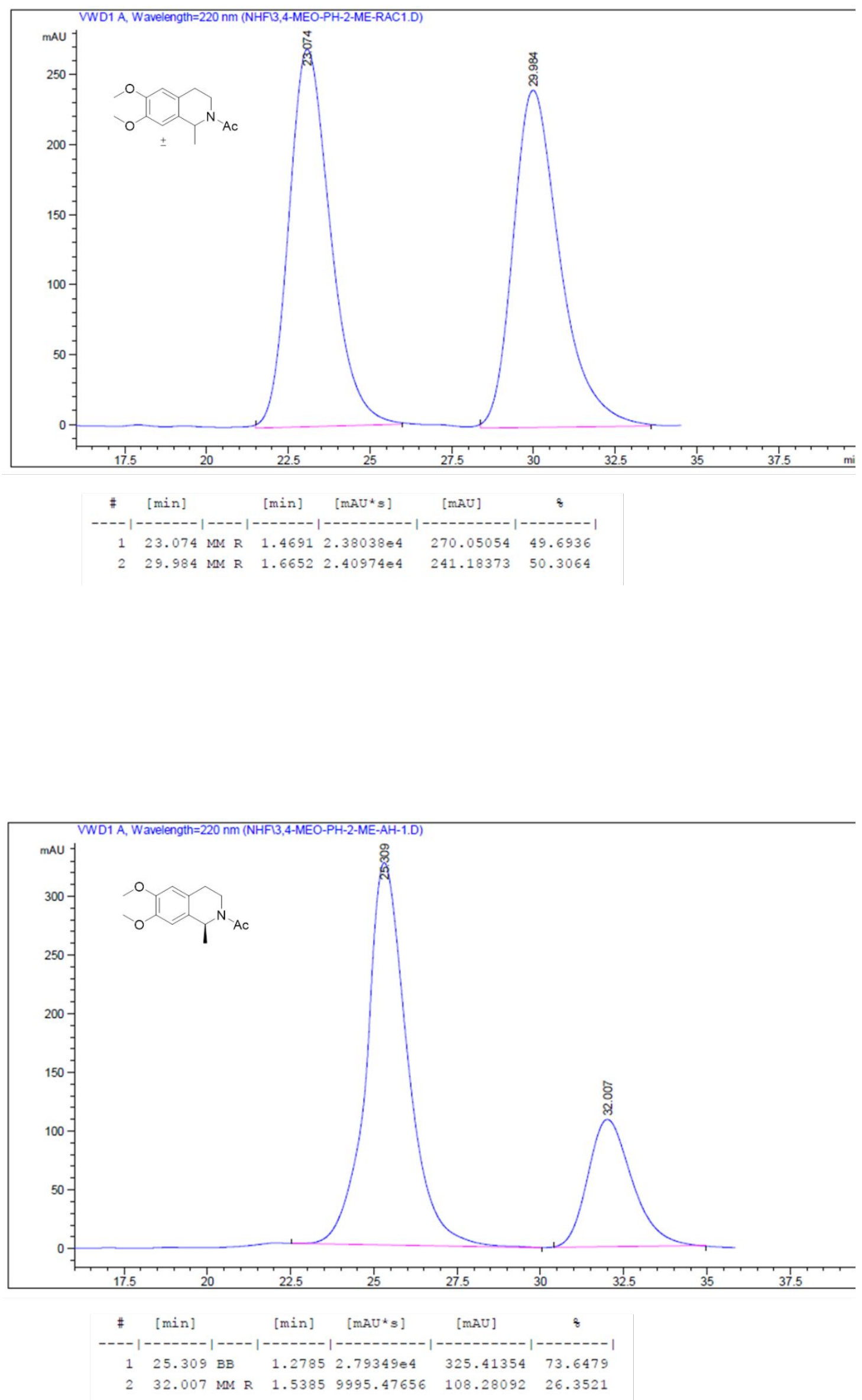

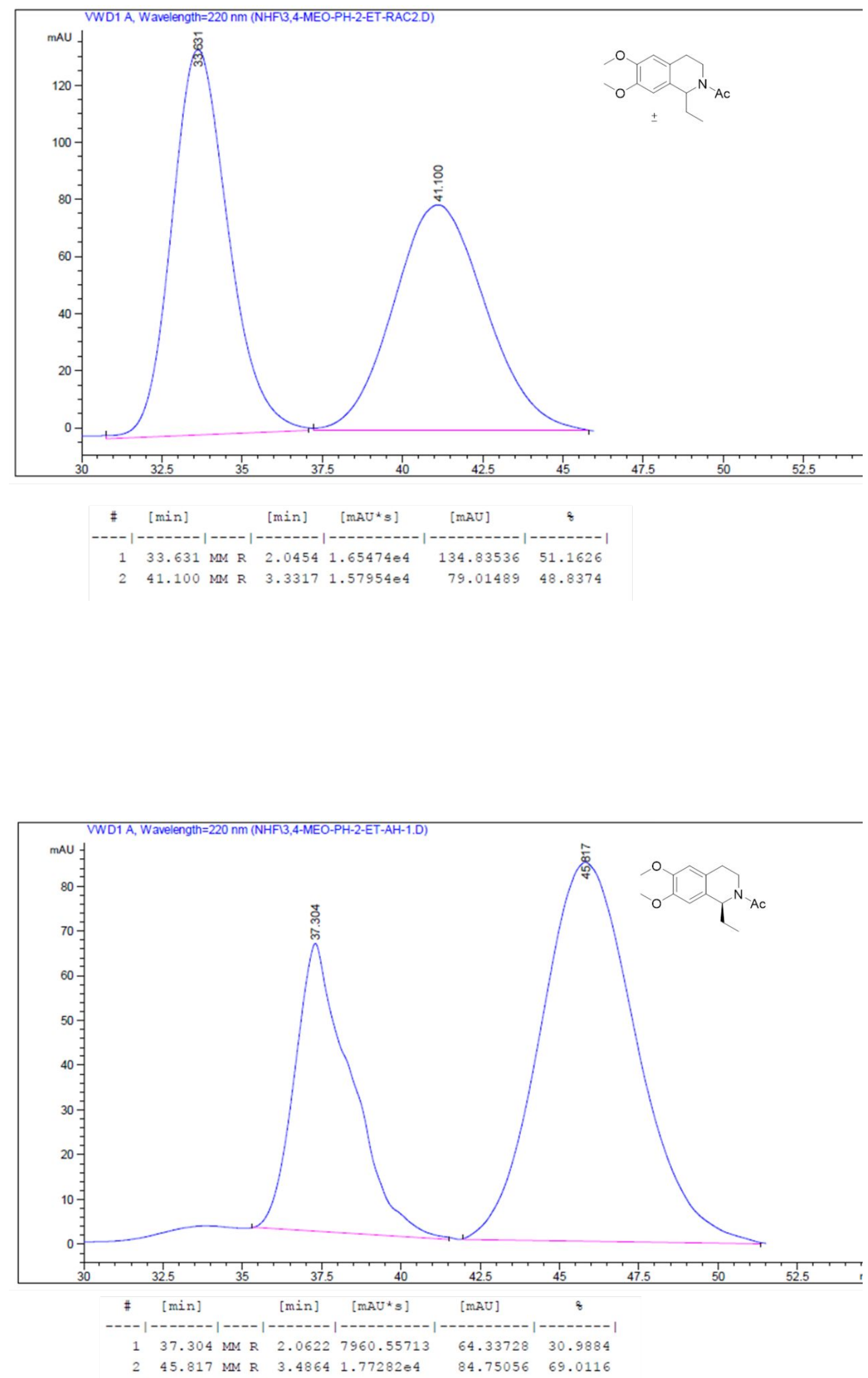


\section{References}

(1) Enthaler, S.; Erre, G.; Junge, K.; Holz, J.; Alberico, E.; Nieddu, I.; Gladiali, S.; Beller, M. Development of Practical Rhodium Phosphine Catalysts for the Hydrogenation of $\beta$-Dehydroamino Acid Derivatives. Org. Process Res. Dev. 2007, 11, 568-577.

(2) Nie, H.; Yao, L.; Li, B.; Zhang, S.; Chen, W. Very Simple and Highly Modular Synthesis of Ferrocene-Based Chiral Phosphines with a Wide Variety of Substituents at the Phosphorus Atom(s). Organometallics 2014, 33, 2109-2114.

(3) Togni, A.; Breutel, C.; Schnyder, A.; Spindler, F.; Landert, H.; Tijani, A. A Novel Easily Accessible Chiral Ferrocenyldiphosphine for Highly Enantioselective Hydrogenation, Allylic Alkylation, and Hydroboration Reactions. J. Am. Chem. Soc. 1994, 116, 4062-4066.

(4) Mohammad, M.; Matthew, D. H. A Versatile Cyclodehydration Reaction for the Synthesis of Isoquinoline and $\beta$-Carboline Derivatives. Org. Lett. 2008, 10, $3485-3488$.

(5) Chang, M.; Li, W.; Zhang, X. A Highly Efficient and Enantioselective Access to Tetrahydroisoquinoline Alkaloids: Asymmetric Hydrogenation with an Iridium Catalyst. Angew. Chem. Int. Ed. 2011, 50, 10679-10681.

(6) Péter, Á. B.; Péter, S.; Balázs, V.; László, P.; Mátyás, M. New Synthetic Approach for the Preparation of 1-Aryl-3,4-dihydroisoquinolines by Liebeskind-Srogl Reaction. Synlett 2014, 25, 2574-2578.

(7) Zhou, H.; Liu, Y.; Yang, S.; Chang, M. One-Pot N-Deprotection and Catalytic Intramolecular Asymmetric Reductive Amination for the Synthesis of Tetrahydroisoquinolines. Angew. Chem. Int. Ed. 2017, 56, 2725-2729.

(8) Perez, M.; Wu, Z.; Scalone, M.; Ayad, T.; Ratovelomanana-Vidal, V. Enantioselective Synthesis of 1-Aryl-Substituted Tetrahydroisoquinolines Through Ru-Catalyzed Asymmetric Transfer Hydrogenation. Eur. J. Org. Chem. 2015, 6503-6514.

(9) Wu, Z.; Perez, M.; Scalone, M.; Ayad, T.; Ratovelomanana-Vidal, V. 
Ruthenium-Catalyzed Asymmetric Transfer Hydrogenation of 1-Aryl- Substituted Dihydroisoquinolines: Access to Valuable Chiral 1-Aryl- Tetrahydroisoquinoline Scaffolds. Angew. Chem. Int. Ed. 2013, 52, 4925-4928.

(10)Santanu, H.; Subhabrata, S. Cerium Chloride Catalyzed, 2-Iodoxybenzoic Acid Mediated Oxidative Dehydrogenation of Multiple Heterocycles at Room Temperature. Eur. J. Org. Chem. 2017, 1277-1280.

(11)Li, H.; Tian, P.; Xu, J.; Zheng, G. Identification of an Imine Reductase for Asymmetric Reduction of Bulky Dihydroisoquinolines. Org. Lett. 2017, 19, $3151-3154$.

(12)Rosaria, G.; Roberta, C.; Valerie, O.; Silvana, Q.; Letizia, B. M.; Guido, F.; Emilio, R.; Giovambattista, De S.; Alba, C. Synthesis and anticonvulsant properties of tetrahydroisoquinoline derivatives. Il Farmaco 2004, 59, 7-12. 